\title{
S3-Leitlinie - Diagnostik und Therapie biliärer Karzinome
}

\author{
Langversion 2.0 - Juni 2021 - AWMF-Registernummer: 032-053OL
}

\section{Autoren}

M. Bitzer ${ }^{1}$, S. Voesch ${ }^{1}$, J. Albert' ${ }^{2}$, P. Bartenstein ${ }^{3}$, W. Bechstein ${ }^{4}$, S. Blödt' ${ }^{5}$, T. Brunner ${ }^{6}$, F. Dombrowski ${ }^{7}$, M. Evert ${ }^{8}$, M. Follmann ${ }^{9}$, C. La Fougère ${ }^{10}$, P. Freudenberger ${ }^{11}$, A. Geier ${ }^{12}$, E. Gkika ${ }^{13}$, M. Götz ${ }^{14}$, E. Hammes ${ }^{15}$, T. Helmberger ${ }^{16}$, R. T. Hoffmann ${ }^{17}$, W. P. Hofmann ${ }^{18}$, P. Huppert ${ }^{19}$, A. Kautz ${ }^{20}$, G. Knötgen ${ }^{21}$, J. Körber ${ }^{22}$, D. Krug ${ }^{23}$, F. Lammert ${ }^{24}$, H. Lang ${ }^{25}$, T. Langer ${ }^{26}$, P. Lenz ${ }^{27}$, A. Mahnken ${ }^{28}$, A. Meining ${ }^{29}$, O. Micke ${ }^{30}$, S. Nadalin ${ }^{31}$, H. P. Nguyen ${ }^{32}$, J. Ockenga ${ }^{33}$, K. Oldhafer $^{34}$, P. Paprottka ${ }^{35}$, K. Paradies ${ }^{36}$, P. Pereira ${ }^{37}$, T. Persigehl ${ }^{38}$, M. Plauth ${ }^{39}$, R. Plentz ${ }^{40}$, J. Pohl ${ }^{41}$, J. Riemer ${ }^{42}$, P. Reimer ${ }^{43}$, J. Ringwald ${ }^{44}$, U. Ritterbusch ${ }^{45}$, E. Roeb ${ }^{46}$, B. Schellhaas ${ }^{47}$, P. Schirmacher ${ }^{48}$, I. Schmid ${ }^{49}$, A. Schuler ${ }^{50}$, D. von Schweinitz ${ }^{51}$, D. Seehofer ${ }^{52}$, M. Sinn ${ }^{53}$, A. Stein ${ }^{54}$, A. Stengel ${ }^{55}$, N. Steubesand ${ }^{56}$, C. Stoll ${ }^{57}$, A. Tannapfel ${ }^{58}$, A. Taubert ${ }^{59}$, J. Trojan ${ }^{60}$, I. van Thiel ${ }^{61}$, R. Tholen ${ }^{62}$, A. Vogel ${ }^{63}$, T. Vogl ${ }^{64}$, H. Vorwerk ${ }^{65}$, F. Wacker ${ }^{66}$, O. Waidmann ${ }^{67}$, H. Wedemeyer ${ }^{68}$, H. Wege $^{69}$, D. Wildner ${ }^{70}$, C. Wittekind ${ }^{71}$, M. A. Wörns ${ }^{72}$, P. Galle ${ }^{72}$, N. Malek ${ }^{73}$

Institute

1 Medizinische Klinik I, Universitätsklinikum Tübingen

2 Abteilung für Gastroenterologie, Hepatologie und Endokrinologie, Robert-Bosch-Krankenhaus, Stuttgart

3 Klinik und Poliklinik für Nuklearmedizin, LMU Klinikum, München

4 Klinik für Allgemein-, Viszeral-, Transplantations- und Thoraxchirurgie, Universitätsklinikum Frankfurt

5 AWMF-Geschäftsstelle, Berlin

6 Klinik für Strahlentherapie, Universitätsklinikum Magdeburg 7 Institut für Pathologie, Universitätsmedizin Greifswald 8 Institut für Pathologie, Regensburg

9 Office des Leitlinienprogrammes Onkologie, c/o Deutsche Krebsgesellschaft e.V., Berlin

10 Nuklearmedizin und Klinische Molekulare Bildgebung, Tübingen

11 Clinical Guideline Services GmbH, Berlin

12 Medizinische Klinik und Poliklinik II, Universitätsklinikum Würzburg

13 Klinik für Strahlenheilkunde, Department für Radiologische Diagnostik und Therapie, Universitätsklinikum Freiburg

14 Kliniken Böblingen

15 Lebertransplantierte Deutschland e. V., Ansbach

16 Institut für Radiologie, Neuroradiologie und minimal-invasive Therapie, München Klinik Bogenhausen, München

17 Institut und Poliklinik für Diagnostische und Interventionelle Radiologie, Dresden

18 Gastroenterologie am Bayerischen Platz, medizinisches Versorgungszentrum, Berlin

19 Radiologisches Zentrum, Max Grundig Klinik, Bühl

20 Deutsche Leberhilfe e.V., Köln

21 Konferenz onkologischer Kranken- und Kinderkrankenpflege, Hamburg

22 Klinik Nahetal, Fachklinik für onkologische Rehabilitation und Anschlussrehabilitation, Bad Kreuznach

23 Klinik für Strahlentherapie, Universitätsklinikum Schleswig-Holstein, Kiel

24 Medizinische Hochschule Hannover
25 Klinik für Allgemein-, Viszeral und Transplantationschirurgie, Universitätsmedizin der Johannes Gutenberg-Universität Mainz

26 Office des Leitlinienprogrammes Onkologie, c/o Deutsche Krebsgesellschaft e.V., Berlin

27 Universitätsklinikum Münster, Zentrale Einrichtung Palliativmedizin, Münster

28 Klinik für Diagnostische und Interventionelle Radiologie, Universitätsklinikum Gießen und Marburg GmbH, Marburg

29 Medizinische Klinik und Poliklinik II des Universitätsklinikums Würzburg

30 Klinik für Strahlentherapie und Radioonkologie, Franziskus Hospital Bielefeld

31 Universitätsklinik für Allgemein-, Viszeral- und Transplantationschirurgie, Universitätsklinikum Tübingen

32 Humangenetik, Ruhr-Universität, Bochum

33 Medizinische Klinik II, Klinikum Bremen-Mitte, Bremen

34 Klinik für Leber-, Gallenwegs- und Pankreaschirurgie, Semmelweis Universität, Asklepios Campus Hamburg

35 Abteilung für interventionelle Radiologie, Klinikum rechts der Isar der Technischen Universität München

36 Konferenz onkologischer Kranken- und Kinderkrankenpflege, Hamburg

37 Abteilung für interventionelle Radiologie, Klinikum rechts der Isar der Technischen Universität München

38 Institut für Diagnostische und Interventionelle Radiologie, Universitätsklinikum Köln

39 Städtisches Klinikum Dessau

40 Klinikum Bremen-Nord, Innere Medizin, Bremen

41 Interventionelles Endoskopiezentrum und Schwerpunkt Gastrointestinale Onkologie, Asklepios Klinik Altona, Hamburg

42 Lebertransplantierte Deutschland e. V., Bretzfeld

43 Institut für diagnostische und interventionelle Radiologie, Städtisches Klinikum Karlsruhe gGmbH, Karlsruhe

44 Psychosomatische Medizin und Psychotherapie, Universitätsklinikum Tübingen

45 Hospizarbeit am Universitätsklinikum Essen 
46 Medizinische Klinik II, Universitätsklinikum Gießen und Marburg GmbH, Gießen

47 Medizinische Klinik I, Friedrich-Alexander Universität Erlangen-Nürnberg, Erlangen

48 Pathologisches Institut, Universitätsklinikum Heidelberg 49 Zentrum Pädiatrische Hämatologie und Onkologie,

Dr. von Haunersches Kinderspital, Klinikum der Universität München

50 Medizinische Klinik, Alb Fils Kliniken GmbH, Göppingen

51 SRH Wilhelm Löhe Hochschule, Fürth

52 Klinik und Poliklinik für Viszeral-, Transplantations-,

Thorax- und Gefäßchirurgie, Universitätsklinikum Leipzig

53 Medizinische Klinik II, Universitätsklinikum HamburgEppendorf

54 Hämatologisch-Onkologischen Praxis Eppendorf, Hamburg

55 Psychosomatische Medizin und Psychotherapie,

Universitätsklinikum Tübingen

56 Clinical Guideline Services GmbH, Kiel

57 Klinik Herzoghöhe Bayreuth, Bayreuth

58 Institut für Pathologie der Ruhr-Universität Bochum am Berufsgenossenschaftlichen Universitätsklinikum Bergmannsheil, Bochum

59 Kliniksozialdienst, Universitätsklinikum Heidelberg, Bochum

60 Medizinische Klinik I, Universitätsklinikum Frankfurt, Frankfurt am Main

61 Deutsche Leberhilfe e.V., Köln

62 Deutscher Verband für Physiotherapie e. V., Köln

63 Klinik für Gastroenterologie, Hepatologie, Endokrinologie der Medizinischen Hochschule Hannover, Hannover

64 Universitätsklinikum Frankfurt, Institut für Diagnostische und Interventionelle Radiologie, Frankfurt

65 Klinik für Strahlentherapie, Universitätsklinikum Gießen und Marburg GmbH, Marburg
66 Institut für Diagnostische und Interventionelle Radiologie der Medizinischen Hochschule Hannover, Hannover

67 Medizinische Klinik I, Universitätsklinikum Frankfurt, Frankfurt am Main

68 Klinik für Gastroenterologie, Hepatologie und Endokrinologie Medizinische Hochschule Hannover, Hannover

69 Medizinische Klinik und Poliklinik, Universitätsklinikum Hamburg-Eppendorf, Hamburg

70 Innere Medizin, Krankenhäuser Nürnberger Land GmbH, Lauf an der Pegnitz

71 Institut für Pathologie, Universitätsklinikum Leipzig, Leipzig

72 Medizinische Klinik und Poliklinik, Universitätsklinikum Mainz, Mainz

73 Medizinische Klinik I, Universitätsklinikum Tübingen, Tübingen

Bibliografie

Z Gastroenterol 2022; 60: e186-e227

DOI 10.1055/a-1589-7854

ISSN $0044-2771$

(C) 2022. Thieme. All rights reserved.

Georg Thieme Verlag KG, Rüdigerstraße 14,

70469 Stuttgart, Germany

\section{Korrespondenzadresse}

Sabrina Voesch

Medizinische Klinik I

Universitätsklinikum Tübingen, Otfried-Müller-Str. 10,

72076 Tübingen, Deutschland

Sabrina.Voesch@med.uni-tuebingen.de

Nisar Malek

Medizinische Klinik I

Universitätsklinikum Tübingen, Otfried-Müller-Str. 10, 72076 Tübingen, Deutschland

\begin{tabular}{|l|l|l|}
\hline \multicolumn{2}{|l|}{ Inhaltsverzeichnis } & Seite \\
\hline 1.9. & Zusammensetzung der Leitliniengruppe & $\mathrm{e} 189$ \\
\hline 1.9 .1$. & Koordination und Redaktion & $\mathrm{e} 189$ \\
\hline 1.9 .2$. & $\begin{array}{l}\text { Beteiligte Fachgesellschaften } \\
\text { und Organisationen }\end{array}$ & $\mathrm{e} 189$ \\
\hline 1.9 .3$. & Patientenbeteiligung & $\mathrm{e} 190$ \\
\hline 1.9 .4$. & Methodische Begleitung & $\mathrm{e} 190$ \\
\hline 1.10. & Verwendete Abkürzungen & $\mathrm{e} 190$ \\
\hline 2. & Einführung & $\mathrm{e} 193$ \\
\hline 2.1. & Geltungsbereich und Zweck & $\mathrm{e} 193$ \\
\hline 2.1 .1$. & Zielsetzung und Fragestellung & $\mathrm{e} 193$ \\
\hline 2.1 .2$. & Adressaten & $\mathrm{e} 194$ \\
\hline 2.1 .3$. & $\begin{array}{l}\text { Gültigkeitsdauer und } \\
\text { Aktualisierungsverfahren }\end{array}$ & $\mathrm{e} 194$ \\
\hline 2.1 .4$. & Grundlagen der Methodik & $\mathrm{e} 194$ \\
\hline
\end{tabular}

\begin{tabular}{|l|l|l|}
\hline \multicolumn{2}{|l|}{ Inhaltsverzeichnis } & Seite \\
\hline 2.1.5. & $\begin{array}{l}\text { Schema der Evidenzgraduierung nach Oxford } \\
\text { (Version 2011) }\end{array}$ & $\mathrm{e} 194$ \\
\hline 2.1 .6$. & Schema der Empfehlungsgraduierung & $\mathrm{e} 194$ \\
\hline 2.1 .7$. & Statements & $\mathrm{e} 194$ \\
\hline 2.1 .8$. & Expertenkonsens (EK) & $\mathrm{e} 194$ \\
\hline 2.1 .9$. & Klug entscheiden & $\mathrm{e} 194$ \\
\hline 2.1 .10$. & $\begin{array}{l}\text { Unabhängigkeit und Darlegung möglicher } \\
\text { Interessenkonflikte }\end{array}$ & $\mathrm{e} 194$ \\
\hline 3. & $\begin{array}{l}\text { Diagnostik und Therapie des Hepatozellulären } \\
\text { Karzinoms }\end{array}$ & $\mathrm{e} 196$ \\
\hline 4. & $\begin{array}{l}\text { Diagnostik und Therapie der biliären } \\
\text { Karzinome }\end{array}$ & $\mathrm{e} 197$ \\
\hline 4.1. & Risikofaktoren, Prävention & $\mathrm{e} 197$ \\
\hline 4.1 .1$. & Und Früherkennung & Risikofaktoren \\
\hline
\end{tabular}




\begin{tabular}{|c|c|c|}
\hline \multicolumn{2}{|c|}{ Inhaltsverzeichnis } & \multirow{2}{*}{$\begin{array}{l}\text { Seite } \\
\text { e198 }\end{array}$} \\
\hline 4.1.2. & $\begin{array}{l}\text { Prophylaktische und therapeutische } \\
\text { Maßnahmen zur Reduktion des Risikos } \\
\text { der Entstehung von biliären Karzinomen }\end{array}$ & \\
\hline 4.1.3. & Früherkennung & e198 \\
\hline 4.2 . & $\begin{array}{l}\text { Histopathologische und molekulare } \\
\text { Diagnostik }\end{array}$ & e199 \\
\hline 4.2.1. & $\begin{array}{l}\text { Typisierung und Staging von biliären } \\
\text { Karzinomen }\end{array}$ & e199 \\
\hline 4.2.2. & $\begin{array}{l}\text { Zytologische und histopathologische } \\
\text { Untersuchungen zur Diagnostik eines CCA } \\
\text { oder eines Gallenblasenkarzinoms }\end{array}$ & e201 \\
\hline 4.2.3. & Molekulare Diagnostik & e202 \\
\hline 4.3. & Bildgebende und endoskopische Diagnostik & e203 \\
\hline 4.3.1. & $\begin{array}{l}\text { Bildgebende und/oder endoskopische } \\
\text { Untersuchungen zum Staging und zur } \\
\text { Diagnosestellung eines biliären Karzinoms }\end{array}$ & e203 \\
\hline 4.3.2. & $\begin{array}{l}\text { Untersuchungsmethoden zur Darstellung } \\
\text { der maximalen Ausbreitung des Tumors }\end{array}$ & e205 \\
\hline 4.3.3. & Diagnostikalgorithmus & e205 \\
\hline 4.3.4. & Endoskopische Diagnostik & e205 \\
\hline 4.4 . & $\begin{array}{l}\text { Operative und interventionelle } \\
\text { Therapieverfahren }\end{array}$ & e207 \\
\hline 4.4.1. & Resektion & e207 \\
\hline 4.4.2. & Lebertransplantation & e209 \\
\hline 4.4.3. & Interventionelle Therapieverfahren & e210 \\
\hline 4.4.3.1. & Perkutane Ablation & e210 \\
\hline 4.4.3.2. & Intraaterielle Therapieverfahren & e210 \\
\hline 4.4.4. & Endoskopische Therapieverfahren & e211 \\
\hline 4.4.4.1. & Präoperative biliäre Drainagen & e211 \\
\hline 4.4.4.2. & Palliative biliäre Drainage & e212 \\
\hline 4.4.4.3. & Intraduktale lokoregionäre Therapieverfahren & e213 \\
\hline 4.4.5. & Stereotaxie & e213 \\
\hline 4.4.6. & Nachsorge & e214 \\
\hline 4.5 . & Systemtherapie & e214 \\
\hline 4.5.1. & Adjuvante Therapie & e214 \\
\hline 4.5.2. & $\begin{array}{l}\text { Neoadjuvante Therapie primär resektabler } \\
\text { Tumoren }\end{array}$ & e215 \\
\hline 4.5.3. & $\begin{array}{l}\text { Systemtherapie lokal fortgeschrittener } \\
\text { Tumoren }\end{array}$ & e215 \\
\hline 4.5.4. & Palliative Systemtherapie & e215 \\
\hline 4.5.4.1. & Erstlinientherapie & e215 \\
\hline 4.5.4.2. & $\begin{array}{l}\text { Therapie nach Versagen } \\
\text { der Erstlinientherapie }\end{array}$ & e216 \\
\hline 4.5.5. & Verlaufskontrollen unter Systemtherapie & e217 \\
\hline 4.6. & $\begin{array}{l}\text { Supportivtherapie des Hepatozellulären } \\
\text { Karzinoms und der biliären Karzinome }\end{array}$ & e217 \\
\hline 5. & Qualitätsindikatoren & e217 \\
\hline 6. & Anhang & e218 \\
\hline 6.1. & Literaturübersichten & e218 \\
\hline
\end{tabular}

\begin{tabular}{|l|l|l|}
\multicolumn{2}{l}{ Inhaltsverzeichnis } & Seite \\
\hline 6.1.2. & $\begin{array}{l}\text { Kapitel 4.4. Operative und Interventionelle } \\
\text { Therapie der biliären Karzinome }\end{array}$ & e218 \\
\hline 7. & Abbildungsverzeichnis & e220 \\
\hline 8. & Tabellenverzeichnis & e220 \\
\hline & Literaturverzeichnis & e220 \\
\hline
\end{tabular}

\section{Informationen zu dieser Leitlinie}

\subsection{Herausgeber}

Leitlinienprogramm Onkologie der Arbeitsgemeinschaft der Wissenschaftlichen Medizinischen Fachgesellschaften e. V. (AWMF), der Deutschen Krebsgesellschaft e. V. (DKG) und der Deutschen Krebshilfe (DKH).

\subsection{Federführende Fachgesellschaft(en)}

Deutsche Gesellschaft für Gastroenterologie, Verdauungs- und Stoffwechselkrankheiten

\section{${ }^{193}$ DGVS \\ Deutsche Gesellschaft für Gastroenterologie, Verdauungs- und Stoffwechselkrankheiten}

\subsection{Finanzierung der Leitlinie}

Diese Leitlinie wurde von der Deutschen Krebshilfe im Rahmen des Leitlinienprogramms Onkologie gefördert.

\subsection{Kontakt}

Office Leitlinienprogramm Onkologie c/o Deutsche Krebsgesellschaft e. V. Kuno-Fischer-Straße 8 14057 Berlin leitlinienprogramm@krebsgesellschaft.de www.leitlinienprogramm-onkologie.de

\subsection{Zitierweise}

Leitlinienprogramm Onkologie (Deutsche Krebsgesellschaft, Deutsche Krebshilfe, AWMF): Diagnostik und Therapie des Hepatozellulären Karzinoms und biliärer Karziome, Langversion 2.0, Juni 2021, AWMF Registernummer: 032/-0530L, https://www. leitlinienprogramm-onkologie.de/leitlinien/hepatozellulaereskarzinom-hcc/ (Zugriff am: TT.MM.JJJJ) 


\subsection{Besonderer Hinweis}

Die Medizin unterliegt einem fortwährenden Entwicklungsprozess, sodass alle Angaben, insbesondere zu diagnostischen und therapeutischen Verfahren, immer nur dem Wissensstand zur Zeit der Drucklegung der Leitlinie entsprechen können. Hinsichtlich der angegebenen Empfehlungen zur Therapie und der Auswahl sowie Dosierung von Medikamenten wurde die größtmögliche Sorgfalt beachtet. Gleichwohl werden die Benutzer aufgefordert, die Beipackzettel und Fachinformationen der Hersteller zur Kontrolle heranzuziehen und im Zweifelsfall einen Spezialisten zu konsultieren. Fragliche Unstimmigkeiten sollen bitte im allgemeinen Interesse der OL-Redaktion mitgeteilt werden.

Der Benutzer selbst bleibt verantwortlich für jede diagnostische und therapeutische Applikation, Medikation und Dosierung.

In dieser Leitlinie sind eingetragene Warenzeichen (geschützte Warennamen) nicht besonders kenntlich gemacht. Es kann also aus dem Fehlen eines entsprechenden Hinweises nicht geschlossen werden, dass es sich um einen freien Warennamen handelt.

Das Werk ist in allen seinen Teilen urheberrechtlich geschützt. Jede Verwertung außerhalb der Bestimmung des Urhebergesetzes ist ohne schriftliche Zustimmung der OL-Redaktion unzulässig und strafbar. Kein Teil des Werks darf in irgendeiner Form ohne schriftliche Genehmigung der OL-Redaktion reproduziert werden. Dies gilt insbesondere für Vervielfältigungen, Übersetzungen, Mikroverfilmungen und die Einspeicherung, Nutzung und Verwertung in elektronischen Systemen, Intranets und dem Internet.

In dieser Leitlinie wird aus Gründen der Lesbarkeit die männliche Form verwendet, dessenungeachtet beziehen sich die Angaben auf Angehörige aller Geschlechter.

\subsection{Ziele des Leitlinienprogramms Onkologie}

Die Arbeitsgemeinschaft der Wissenschaftlichen Medizinischen Fachgesellschaften e. V., die Deutsche Krebsgesellschaft e. V. und die Deutsche Krebshilfe haben sich mit dem Leitlinienprogramm Onkologie (OL) das Ziel gesetzt, gemeinsam die Entwicklung und Fortschreibung und den Einsatz wissenschaftlich begründeter und praktikabler Leitlinien in der Onkologie zu fördern und zu unterstützen. Die Basis dieses Programms beruht auf den medizinisch-wissenschaftlichen Erkenntnissen der Fachgesellschaften und der DKG, dem Konsens der medizinischen Fachexperten, Anwender und Patienten sowie auf dem Regelwerk für die Leitlinienerstellung der AWMF und der fachlichen Unterstützung und Finanzierung durch die Deutsche Krebshilfe. Um den aktuellen Stand des medizinischen Wissens abzubilden und den medizinischen Fortschritt zu berücksichtigen, müssen Leitlinien regelmäßig überprüft und fortgeschrieben werden. Die Anwendung des AWMF-Regelwerks soll dabei Grundlage zur Entwicklung qualitativ hochwertiger onkologischer Leitlinien sein. Da Leitlinien ein wichtiges Instrument der Qualitätssicherung und des Qualitätsmanagements in der Onkologie darstellen, sollten sie gezielt und nachhaltig in den Versorgungsalltag eingebracht werden. So sind aktive Implementierungsmaßnahmen und auch Evaluationsprogramme ein wichtiger Bestandteil der Förderung des Leitlinienprogramms Onkologie. Ziel des Programms ist es, in Deutschland professionelle und mittelfristig finanziell gesicherte Vorausset- zungen für die Entwicklung und Bereitstellung hochwertiger Leitlinien zu schaffen. Diese hochwertigen Leitlinien dienen nicht nur dem strukturierten Wissenstransfer, sondern können auch in der Gestaltung der Strukturen des Gesundheitssystems ihren Platz finden. Zu erwähnen sind in diesem Zusammenhang evidenzbasierte Leitlinien als Grundlage zum Erstellen und Aktualisieren von Disease-Management-Programmen oder die Verwendung von aus Leitlinien extrahierten Qualitätsindikatoren im Rahmen der Zertifizierung von Organtumorzentren.

\subsection{Weitere Dokumente zu dieser Leitlinie}

Bei diesem Dokument handelt es sich um die Langversion der S3-Leitlinie „Diagnostik und Therapie des Hepatozellulären Karzinoms und biliärer Karzinome“. Neben der Langversion wird es folgende ergänzende Dokumente zu dieser Leitlinie geben:

- Kurzversion der Leitlinie

- Laienversion (Patientenleitlinie)

- Leitlinienreport zum Erstellungsprozess der Leitlinie

- Evidenztabellen

Diese Leitlinie und alle Zusatzdokumente sind über die folgenden Seiten zugänglich.

- Leitlinienprogramm Onkologie (https://www.leitlinienpro gramm-onkologie.de/leitlinien/hepatozellulaeres-karzinomhCc/)

- AWMF (www.leitlinien.net)

- Deutsche Gesellschaft für Gastroenterologie, Verdauungs- und Stoffwechselkrankheiten (www.dgvs.de)

- Guidelines International Network (www.g-i-n.net)

Die Leitlinie ist außerdem in der App des Leitlinienprogramms Onkologie enthalten.

Weitere Informationen unter: https://www.leitlinienprogrammonkologie.de/app/

\subsection{Zusammensetzung der Leitliniengruppe}

\subsubsection{Koordination und Redaktion}

Prof. Dr. Nisar P. Malek

Ärztlicher Direktor Medizinische Klinik Universitätsklinikum Tübingen

Prof. Dr. Michael Bitzer

Stellvertretener Ärztlicher Direktor Medizinische Klinik Universitätsklinikum Tübingen

Prof. Dr. Peter R. Galle

Ärztlicher Direktor Universitätsmedizin der Johannes GutenbergUniversität Mainz

Sabrina Voesch

Ärztin in Weiterbildung Medizinische Klinik Universitätsklinikum Tübingen

\subsubsection{Beteiligte Fachgesellschaften und Organisationen}

In $\triangleright$ Tab. 1 sind die an der Leitlinienerstellung beteiligten medizinischen Fachgesellschaften und sonstigen Organisationen sowie deren mandatierte Vertreter aufgeführt. 
Außerdem wurden folgende Fachgesellschaften für den Leitlinienprozess angeschrieben, diese haben jedoch keinen Mandatsträger benannt:

- Deutsche Gesellschaft für Allgemeinmedizin und Familienmedizin

- Deutsche Gesellschaft für Pflegewissenschaft e. V.

- Arbeitsgemeinschaft Pädiatrische Onkologie

- Arbeitsgemeinschaft Onkologische Thoraxchiurgie

- Deutsche Gesellschaft für Ernährung e. V.

- Deutsche Gesellschaft für Klinische Chemie und Laboratoriumsmedizin ( $>$ Tab. 2, 3)

Die Zuordnung der beteiligten Mandatsträger und Experten finden Sie in den $>$ Tab. 2, 3.

\subsubsection{Patientenbeteiligung}

Die Leitlinie wurde unter direkter Beteiligung von 4 Patientenvertretern erstellt.

Herr Ingo van Thiel, Herr Achim Kautz und Frau Elke Hammes waren von Beginn an in die Erstellung der Leitlinie eingebunden und nahmen mit eigenem Stimmrecht an der Konsensuskonferenz teil. Herr Kautz war der Stellvertreter von Herrn Thiel und hat daher nicht abgestimmt. Frau Riemer ersetzte Frau Hammes ab der Videokonsensuskonferenz 08/2020.

\subsubsection{Methodische Begleitung}

1. Durch das Leitlinienprogramm Onkologie:

a) Dr. med. Markus Follmann, MPH, MSc (OL Office c/o Deutsche Krebsgesellschaft)

b) Thomas Langer, Dipl.-Soz.-Wiss. (OL Office c/o Deutsche Krebsgesellschaft)

2. Durch die Arbeitsgemeinschaft der Wissenschaftlichen Medizinischen Fachgesellschaften e. V.:

a) Dr. rer. medic. Susanne Blödt, MScPH (AWMF-IMWI)

3. Durch die Firma Clinical Guideline Service - User Group:

a) Dr. Nadine Steubesand

b) Dr. Paul Freudenberger

4. Durch die DGVS

a) Pia Lorenz

b) PD Dr. med. Petra Lynen Jansen

\subsection{Verwendete Abkürzungen}

\begin{tabular}{|l|l|}
\hline Abkürzung & Erläuterung \\
\hline 3D-CRT & Three-dimensional Conformal Radiation Therapy \\
\hline 5 -FU & 5-Fluorouracil \\
\hline AASLD & American Association for the Study of Liver Diseases \\
\hline ACG & American College of Gastroenterology \\
\hline AFIP & Armed Forces Institute of Pathology \\
\hline AFP & Alpha-Fetoprotein \\
\hline AFP-L3 & Lektin reaktives Alpha-Fetoprotein \\
\hline AG & Arbeitsgruppe \\
\hline ALT & Alanin-Aminotransferase \\
\hline APASL & Asian Pacific Association for the Study of the Liver \\
\hline
\end{tabular}

\begin{tabular}{|c|c|}
\hline Abkürzung & Erläuterung \\
\hline APRI & AST/Thrombozyten-Ratio-Index \\
\hline ARFI & Acoustic Radiation Force Impulse Imaging \\
\hline AST & Aspartat-Aminotransferase \\
\hline ATG & Antithymozytenglobulin \\
\hline AUC & Area under the Curve \\
\hline AWMF & $\begin{array}{l}\text { Arbeitsgemeinschaft der Wissenschaftlichen } \\
\text { Medizinischen Fachgesellschaften e. V. }\end{array}$ \\
\hline$\ddot{A} Z Q$ & Ärztliches Zentrum für Qualität in der Medizin \\
\hline BÄK & Bundesärztekammer \\
\hline $\mathrm{BCLC}$ & Barcelona Clinic Liver Cancer \\
\hline Bds & Beiderseits \\
\hline BillN & Biliäre intraepitheliale Neoplasie \\
\hline BMI & Body-Mass-Index \\
\hline BSC & Best Supportive Care \\
\hline BSG & British Society of Gastroenterology \\
\hline CA 19-9 & Carbohydrate-Antigen 19-9 \\
\hline CCA & Cholangiokarzinom \\
\hline iCCA & Intrahepatisches Cholangiokarzinom \\
\hline dCCA & Distales Cholangiokarzinom \\
\hline eCCA & Extrahepatisches Cholangiokarzinom \\
\hline pCCA & Perihiläres Cholangiokarzinom \\
\hline$C D$ & Cluster of Differentiation \\
\hline CEUS & Kontrastmittel-Ultraschall \\
\hline $\mathrm{Cl}$ & Konfidenzintervall \\
\hline CIPN & Chemotherapie-induzierte periphere Neuropathie \\
\hline CLIP & Cancer of the Liver Italian Program \\
\hline $\mathrm{CNI}$ & Calcineurininhibitor \\
\hline$C R$ & Complete Remission \\
\hline CT & Computertomografie \\
\hline CTCEA & Common Terminology Criteria for Adverse Events \\
\hline CTLA-4 & Cytotoxic T-lymphocyte-associated Protein 4 \\
\hline CU-HCC & Chinese University-HCC (Risikoscore) \\
\hline CUP & Cancer of Unkown Primary \\
\hline DAAD & Direct-acting antiviral Drugs \\
\hline DCP & des-Gamma-Carboxyprothrombin \\
\hline $\mathrm{DGCH}$ & Deutsche Gesellschaft für Chirurgie \\
\hline DGEM & Deutsche Gesellschaft für Ernährungsmedizin \\
\hline DGVS & $\begin{array}{l}\text { Deutsche Gesellschaft für Gastroenterologie, } \\
\text { Verdauungs- und Stoffwechselkrankheiten }\end{array}$ \\
\hline $\mathrm{DHC}$ & Ductus Choledochus \\
\hline DKG & Deutsche Krebsgesellschaft e. V. \\
\hline DKH & Deutsche Krebshilfe e. V. \\
\hline EASL & European Association for the Study of the Liver \\
\hline ECOG & Eastern Cooperative Oncology Group \\
\hline EG & $\begin{array}{l}\text { Empfehlungsgrad, } \mathrm{A}=\text { starke Empfehlung, } \\
\mathrm{B}=\text { Empfehlung, } 0=\text { offene Empfehlung }\end{array}$ \\
\hline
\end{tabular}




\begin{tabular}{|c|c|}
\hline Abkürzung & Erläuterung \\
\hline EK & Expertenkonsens \\
\hline ELTR & European Liver Transplant Registry \\
\hline EORTC & $\begin{array}{l}\text { European Organisation for Research and Treatment } \\
\text { of Cancer }\end{array}$ \\
\hline EORTIC-QLQ & $\begin{array}{l}\text { European Organisation for Research and Treatment } \\
\text { of Cancer - Quality of Life Questionnaire }\end{array}$ \\
\hline EQD2 & Equivalenzdosis von 2 Gy \\
\hline $\operatorname{ERC}(\mathrm{P})$ & $\begin{array}{l}\text { Endoskopische retrograde } \\
\text { Cholangio(pankreatiko)grafie }\end{array}$ \\
\hline ESCULAP & $\begin{array}{l}\text { Erlanger Synopsis for Contrast-enhanced Ultrasound } \\
\text { for Liver Lesion Assessment in Patients at Risk }\end{array}$ \\
\hline ESMO & European Society of Medical Oncology \\
\hline ETC & Extended Toronto Criteria \\
\hline EUS-(FNA) & $\begin{array}{l}\text { Endosonografischer Ultraschall - } \\
\text { (Feinnadelaspiration) }\end{array}$ \\
\hline FACT-H & $\begin{array}{l}\text { Functional Assessment of Cancer Therapy- } \\
\text { Hepatobiliary }\end{array}$ \\
\hline FDG-PET & Fluordeoxyglukose-Positronen-Emissionstomografie \\
\hline FIB-4 & Fibrosis-4 \\
\hline GAG-HCC & $\begin{array}{l}\text { Guide with Age, Gender, HBV DNA, Core Promoter } \\
\text { Mutations and Cirrhosis-HCC }\end{array}$ \\
\hline GB-CA & Gallenblasenkarzinom \\
\hline Gd-DTPA & Gadolinium-Diethylene-Triamine Pentaacetic Acid \\
\hline Gd-EOB-DTPA & $\begin{array}{l}\text { Gadolinium-Ethoxybenzyl-Diethylentriamin- } \\
\text { Penta-Essigsäure }\end{array}$ \\
\hline GEKID & $\begin{array}{l}\text { Gesellschaft der epidemiologischen Krebsregister } \\
\text { in Deutschland e.V. }\end{array}$ \\
\hline G-I-N & Guidelines International Network \\
\hline GLOBOCAN & Global Cancer Incidence, Mortality and Prevalence \\
\hline GOT & Glutamat-Oxalacetat-Transaminase \\
\hline GPT & Glutamat-Pyruvat-Transaminase \\
\hline $\mathrm{HAl}$ & Hepatische arterielle Infusion \\
\hline $\mathrm{HBsAg}$ & Hepatitis-B-surface-Antigen \\
\hline $\mathrm{HBeAg}$ & Hepatitis-B-envelope-Antigen \\
\hline HBV & Hepatitis B \\
\hline HCC & Hepatozelluläres Karzinom \\
\hline $\mathrm{HCV}$ & Hepatitis C \\
\hline HepPar1 & Hepatocyte Paraffin 1 \\
\hline $\mathrm{HR}$ & Hazard Ratio \\
\hline HSP70 & Hitzeschockprotein 70 \\
\hline hTERT & Human Telomerase Reverse Transcriptase \\
\hline ICCR & International Collaboration on Cancer Reporting \\
\hline ICD & $\begin{array}{l}\text { International Statistical Classification of Diseases } \\
\text { and Related Health Problems }\end{array}$ \\
\hline IGRT & Image Guided Radiotherapy \\
\hline ILCA & International Liver Cancer Association \\
\hline IPMN & Intraduktale papillär-muzinösen Neoplasie \\
\hline IRE & Irreversible Elektroporation \\
\hline
\end{tabular}

\begin{tabular}{|c|c|}
\hline Abkürzung & Erläuterung \\
\hline IQWiG & $\begin{array}{l}\text { Institut für Qualität und Wirtschaftlichkeit } \\
\text { im Gesundheitswesen }\end{array}$ \\
\hline ITT & Intention to Treat \\
\hline JIS & Japan Integrated Staging Score \\
\hline KASL & Korean Association for the Study of the Liver \\
\hline LA & Leitlinienadaptation \\
\hline LAASL & $\begin{array}{l}\text { Latin American Association for the Study of the Liver } \\
\text { (LAASL) }\end{array}$ \\
\hline LiMax & Maximum liver function capacity \\
\hline LiRADS & Liver Imaging Reporting and Data System \\
\hline $\mathrm{LL}$ & Leitlinie \\
\hline LoE & Level of Evidence \\
\hline LTx & Lebertransplantation \\
\hline MARS & Molecular-Adsorbent-Recirculating-System \\
\hline MCN & Muzinös-zystische Neoplasie \\
\hline MRCP & Magnetresonanz-Cholangiopankreatikografie \\
\hline $\operatorname{MR}(T)$ & Magnetresonanz(tomografie) \\
\hline MWA & Mikrowellenablation \\
\hline NAFLD & Non-alcoholic fatty Liver Disease \\
\hline NASH & Nichtalkoholische Steatohepatitis \\
\hline $\mathrm{NCl}$ & National Cancer Institute \\
\hline NCCN & The National Comprehensive Cancer Network \\
\hline NGC & National Guideline Clearinghouse \\
\hline NICE & National Institute for Health and Clinical Excellence \\
\hline NRS & Nutrition Risk Screening \\
\hline NT & Nicht transplantabel \\
\hline $\mathrm{OL}$ & Leitlinienprogramm Onkologie \\
\hline $\mathrm{OP}$ & Operation \\
\hline ORN & Osteoradionekrose \\
\hline OS & Overall Survival \\
\hline PBC & Primär biliäre Zirrhose \\
\hline PBD & Präoperative biliäre Drainage \\
\hline PD & Progressive Disease \\
\hline PDT & Photodynamische Therapie \\
\hline $\mathrm{PEI} / \mathrm{PAI}$ & Perkutane Ethanol Injektion \\
\hline PET & Positronen-Emissions-Tomografie \\
\hline PFS & Progression Free Survival \\
\hline PICO & Population Intervention Comparison Outcome \\
\hline PR & Partial Remission \\
\hline PS & Progressive Disease \\
\hline PSC & Primär sklerosierende Cholangitis \\
\hline PV & Portalvene \\
\hline PZK & Patientenzentrierte Kommunikation \\
\hline QI & Qualitätsindikatoren \\
\hline QoL & Quality of Life \\
\hline RFA & Radiofrequenzablation \\
\hline
\end{tabular}




\begin{tabular}{|l|l|}
\hline Abkürzung & Erläuterung \\
\hline IRFA & Intraduktale Radiofrequenzablation \\
\hline RILD & Radiation induced Liver Disease \\
\hline RR & Relatives Risiko \\
\hline SBRT & Stereotactic Body Radiotherapy \\
\hline SD & Stable Disease \\
\hline SEOM & Spanish Society of Medical Oncology \\
\hline SEMS & Selbstexpandierender Metallstent \\
\hline SGA & Subjective Global Assessment \\
\hline SGB & Sozialgesetzbuch \\
\hline SR & Systematische Recherche \\
\hline STIKO & Ständige Impfkomission \\
\hline SVR & Substained Virological Response \\
\hline
\end{tabular}

\begin{tabular}{|l|l|}
\hline Abkürzung & Erläuterung \\
\hline TACE & Transarterielle Chemoembolisation \\
\hline DEB-TACE & Drug-eluting Bead TACE \\
\hline TARE & Transarterielle Radioembolisation \\
\hline TNM & Tumor Nodus Metastase \\
\hline TTD & Time to Deterioration \\
\hline TTP & Time to Progression \\
\hline UICC & Union for International Cancer Control \\
\hline UCSF & University of California, San Francisco \\
\hline UNOS & United Network of Organ Sharing \\
\hline US & Ultraschall \\
\hline VEGF(R) & Vascular Endothelial Growth Factor (Receptor) \\
\hline WHO & Wold Health Organisation \\
\hline
\end{tabular}

- Tab. 1 Beteiligte Fachgesellschaften und Organisationen.

\section{Beteiligte Fachgesellschaften und Organisationen}

Deutsche Gesellschaft für Allgemein- und Viszeralchirurgie (DGAV)

Deutsche Gesellschaft für Chirurgie (DGCH)

Deutsche Gesellschaft für Ernährungsmedizin (DGEM)

Deutsche Gesellschaft für Endoskopie und bildgebende Verfahren (DGE-BV)

Deutsche Gesellschaft für Hämatologie und Onkologie (DGHO)

Deutsche Gesellschaft für Innere Medizin (DGIM)

Deutsche Gesellschaft für interventionelle Radiologie und minimalinvasive Therapie (DeGIR)

Deutsche Gesellschaft für Nuklearmedizin (DGN)

Deutsche Gesellschaft für Pädiatrische Onkologie und Hämatologie (GPOH)

Deutsche Gesellschaft für Palliativmedizin (DGPall)

Deutsche Gesellschaft für Pathologie (DGP)

Berufsverband deutscher Pathologen (BDP)

Deutsche Gesellschaft für Radioonkologie (DEGRO)

Deutsche Gesellschaft für Ultraschall in der Medizin (DEGUM)

Deutscher Verband für Physiotherapie (ZKV)

Deutsche Röntgengesellschaft e. V. (DRG)

Deutsche Gesellschaft für Gastroenterologie, Verdauungs- und Stoffwechselkrankheiten (DGVS)

Deutsche Transplantationsgesellschaft (DTG)

Deutsche Gesellschaft für Humangenetik (GfH)

Deutsche Leberhilfe e. V.

Deutsche Leberstiftung

Lebertransplantierte Deutschland e.V.

Arbeitsgemeinschaft Internistische Onkologie (AIO)

Arbeitsgemeinschaft Onkologische Pathologie

Arbeitsgemeinschaft Radiologische Onkologie (ARO)

Arbeitsgemeinschaft Tumorklassifikation in der Onkologie (ATO)

\section{Mandatsträger}

Oldhafer K., Seehofer D. (Stellv.)

Lang $\mathrm{H}$.

Plauth M., Ockenga J. (Stellv.)

Meining A.

Sinn M.

Bitzer M., Trojan J.

Pereira P., Mahnken A. (Stellv.), Huppert P. (Stellv.)

Bartenstein P., La Fougère Ch. (Stellv.)

Schmid I., v. Schweinitz D. (Stellv.)

Lenz P.

Schirmacher P., Evert M.

Schirmacher P., Evert M.

Vorwerk, H., Krug D. (Stellv.)

Schuler, A., Wildner D. (Stellv.)

Tholen, $R$.

Vogl, T., Paprottka P., Wacker F., Helmberger T.

Malek N., Galle P., Götz M., Lammert F., Plentz R.

Nadalin S.

Nguyen H. P.

van Thiel I., Kautz A. (Stellv.)

Schirmacher $\mathrm{P}$.

Hammes E. bis 10.08.2020

Riemer J. ab 11.08.2020

Vogel A., Wege $\mathrm{H}$.

Dombrowski F.

Brunner T., Gkika E. (Stellv.)

Tannapfel A., Wittekind Ch. (Stellv.) 
- Tab. 1 (Fortsetzung)

\section{Beteiligte Fachgesellschaften und Organisationen}

Arbeitsgemeinschaft Supportive Maßnahmen in der Onkologie (AGSMO)

Arbeitsgemeinschaft Onkologische Rehabilitation und Sozialmedizin (AGORS)

Chirurgische Arbeitsgemeinschaft Onkologie (CAO-V)

Arbeitsgemeinschaft Psychoonkologie (PSO)

Konferenz Onkologischer Kranken- und Kinderkrankenpflege (KOK)

Arbeitsgemeinschaft Palliativmedizin (APM)

Arbeitsgemeinschaft Bildgebung in der Onkologie (ABO)

Arbeitsgemeinschaft Prävention und Integrative Medizin in der Onkologie (AG PRIO)

Arbeitsgemeinschaft Soziale Arbeit in der Onkologie (ASO)
Mandatsträger

Stein A.

Körber J.

Bechstein W.

Stengel, A., Ringwald J. (Stellv.)

Knötgen G., Paradies K. (Stellv.)

Ritterbusch U.

Persigehl Th.

Stoll Ch., Micke O. (Stellv.)

Taubert A.
- Tab. 2 Arbeitsgruppen und deren Mitglieder.

\begin{tabular}{|l|l|}
\hline Arbeitsgruppe & $\begin{array}{l}\text { Mitglieder der Arbeitsgruppe } \\
\text { (AG-Leiter hervorgehoben) }\end{array}$ \\
\hline $\begin{array}{l}\text { AG I. Risikofaktoren, } \\
\text { Prävention und } \\
\text { Früherkennung }\end{array}$ & $\begin{array}{l}\text { Trojan J., Schuler A., van Thiel I., Kautz A., } \\
\text { Wedemeyer H., Lammert F., Roeb E., } \\
\text { Geier A., Wildner D., Hofmann, W. P., } \\
\text { Schmid I. }\end{array}$ \\
\hline $\begin{array}{l}\text { AG III Histopathologi- } \\
\text { sche und molekulare } \\
\text { Diagnostik }\end{array}$ & $\begin{array}{l}\text { Schirmacher P, Nguyen H. P., } \\
\text { Dombrowski F., Evert M., Tannapfel A. }\end{array}$ \\
\hline $\begin{array}{l}\text { AG II.II Bildgebende } \\
\text { Diagnostik }\end{array}$ & $\begin{array}{l}\text { Götz M., Paprottka P., Persigehl T., } \\
\text { Vogl T.J., Meining A., Wacker F., Pohl J., } \\
\text { Reimer P., Schellhaas B. }\end{array}$ \\
\hline $\begin{array}{l}\text { AG III.I Operative } \\
\text { und Interventionelle } \\
\text { Therapieverfahren }\end{array}$ & $\begin{array}{l}\text { Nadalin S., Pereira P., Bechstein W., } \\
\text { Oldhafer K., Lang H., Bartenstein P., } \\
\text { Vorwerk H., Brunner T., Hammes E., } \\
\text { Vogel A., Helmberger T., Seehofer D., } \\
\text { La Fougère Ch., Albert J., Hoffmann R.-T., } \\
\text { Mahnken A., Huppert P., Gkika E., Krug D. }\end{array}$ \\
\hline AG III.II Systemtherapie & $\begin{array}{l}\text { Bitzer M., Galle P., Sinn M., Stein A., Plentz } \\
\text { R., Schmid I., Wörns M.-A., Wege H. }\end{array}$ \\
\hline AG IV Supportivtherapie & $\begin{array}{l}\text { Lenz P., Ritterbusch U., Tholen R., } \\
\text { Körber J., Stoll Ch., Taubert A., Stengel A., } \\
\text { Knötgen G., Plauth M., Waidmann O. }\end{array}$ \\
\hline
\end{tabular}

\section{Einführung}

\subsection{Geltungsbereich und Zweck}

\subsubsection{Zielsetzung und Fragestellung}

Die interdisziplinäre S3-Leitlinie ist ein Instrument, um die Diagnostik und Therapie des Hepatozellulären Karzinoms (HCC), des Cholangiokarzinoms (CCA) und des Gallenblasenkarzinoms zu verbessern. Fachgruppen aller Disziplinen, die Patienten mit chronischen Lebererkrankungen, Verdacht auf oder bereits diagnostizierten hepatobiliäre Tumoren ambulant und/oder stationär
- Tab. 3 Beteiligte Experten.

\begin{tabular}{|l|l|}
\hline Beteiligte Experten & Arbeitsgruppe \\
\hline Wedemeyer, H. & $\begin{array}{l}\text { AG I Risikofaktoren, Prävention } \\
\text { und Früherkennung }\end{array}$ \\
\hline Roeb, E. & $\begin{array}{l}\text { AG I Risikofaktoren, Prävention } \\
\text { und Früherkennung }\end{array}$ \\
\hline Geier, A. & $\begin{array}{l}\text { AG I Risikofaktoren, Prävention } \\
\text { und Früherkennung }\end{array}$ \\
\hline Pohl, J. & AG II. II Bildgebende Diagnostik \\
\hline Reimer, P. & AG II. II Bildgebende Diagnostik \\
\hline Schellhaas, B. & AG II. II Bildgebende Diagnostik \\
\hline Albert, J. & $\begin{array}{l}\text { AG III.I. Operative und Interventionelle } \\
\text { Therapieverfahren }\end{array}$ \\
\hline Hoffmann, R.-T. & AG III.I. Operative und Interventionelle \\
& Therapieverfahren \\
\hline Hofmann, W. P. & $\begin{array}{l}\text { AG I Risikofaktoren, Prävention } \\
\text { und Früherkennung }\end{array}$ \\
\hline Wörns, M.-A. & AG III.II. Systemtherapie \\
\hline Waidmann, O. & AG IV. Supportivtherapie \\
\hline
\end{tabular}

behandeln, sollen durch die Leitlinie unterstützt werden. Die Leitlinie soll dazu beitragen, eine angemessene Gesundheitsversorgung dieser Patientengruppen sicherzustellen. Es ist weiterhin die Aufgabe der Leitlinie, betroffenen Patienten angemessene, wissenschaftlich begründete und aktuelle Verfahren in der Diagnostik, Therapie und Rehabilitation anzubieten. Dies gilt sowohl für lokal begrenzte oder lokal fortgeschrittene Erkrankungen als auch bei Vorliegen eines Rezidivs oder von Fernmetastasen. Die Leitlinie soll neben dem Beitrag für eine angemessene Gesundheitsversorgung auch die Basis für eine individuell zugeschnittene, qualitativ hochwertige und kosteneffiziente Therapie bieten. Mittel- und langfristig sollen so die Morbidität und die Mortalität von Patienten mit hepatobiliären Tumoren gesenkt und die Lebensqualität erhöht werden. 


\subsubsection{Adressaten}

Die Anwenderzielgruppe sind Ärztinnen und Ärzte aller Versorgungsbereiche (insbesondere Fachärztinnen und Fachärzte), die Patienten mit chronischen Lebererkrankungen, Verdacht auf sowie diagnostizierten hepatobiliären Tumoren ambulant und/ oder stationär behandeln. Dazu gehören unter anderem Allgemeinmediziner, Internisten, Gastroenterologen und Hepatologen, Onkologen, Radiologen, Chirurgen, Palliativmediziner, Pathologen, Nuklearmediziner und Strahlentherapeuten, Psychoonkologen, onkologisch tätige Pflegekräfte und Physiotherapeuten sowie alle an einem HCC oder einem biliären Karzinom erkrankten Patienten und deren Angehörige. Weitere Adressaten dieser Leitlinie sind übergeordnete Organisationen (z. B. Krankenkassen). Sie soll entsprechend der Definition einer Leitlinie Entscheidungshilfen geben, jedoch keine Richtlinie sein. Der behandelnde Arzt ist weiterhin verpflichtet, unter Würdigung der Gesamtsituation des Patienten und mit diesem gemeinsam die für die individuelle Situation angemessene Vorgehensweise zu finden.

\subsubsection{Gültigkeitsdauer und Aktualisierungsverfahren}

Die S3-Leitlinie ist bis zur nächsten Aktualisierung gültig, die Gültigkeitsdauer wird auf 3 Jahre geschätzt. Vorgesehen sind regelmäßige Aktualisierungen, bei dringendem Änderungsbedarf werden diese gesondert publiziert. Kommentare und Hinweise für den Aktualisierungsprozess sind ausdrücklich erwünscht und können an die folgende Adresse gesendet werden: hcc@leitlinien programm-onkologie.de

\subsubsection{Grundlagen der Methodik}

Die methodische Vorgehensweise bei der Erstellung der Leitlinie ist im Leitlinienreport dargelegt. Dieser ist im Internet z. B. auf den Seiten des Leitlinienprogramms Onkologie (http://leitlinien programm-onkologie.de/Leitlinien.7.0.html) und den Seiten der AWMF (http://www.awmf.org/) frei verfügbar.

\subsubsection{Schema der Evidenzgraduierung nach Oxford (Version 2011)}

Zur Klassifikation des Verzerrungsrisikos der identifizierten Studien wurde in dieser Leitlinie das in $>$ Tab. 4 aufgeführte System des Oxford Center for Evidence-based Medicine in der Version von 2011 verwendet. Dieses System sieht die Klassifikation der Studien für verschiedene klinische Fragestellungen (Nutzen von Therapie, prognostische Aussagekraft, diagnostische Wertigkeit) vor.

\subsubsection{Schema der Empfehlungsgraduierung}

Die Methodik des Leitlinienprogramms Onkologie sieht eine Vergabe von Empfehlungsgraden durch die Leitlinienautoren im Rahmen eines formalen Konsensusverfahrens vor. Dementsprechend wurden durch die AWMF moderierte, nominale Gruppenprozesse bzw. strukturierte Konsensuskonferenzen durchgeführt [2]. Im Rahmen dieser Prozesse wurden die Empfehlungen von den stimmberechtigten Mandatsträgern (siehe Kapitel 1.9.2) formal abgestimmt. Die Ergebnisse der jeweiligen Abstimmungen (Kon- sensstärke) sind entsprechend den Kategorien in $\downarrow$ Tab. 6 den Empfehlungen zugeordnet.

In der Leitlinie werden zu allen evidenzbasierten Statements (siehe Kapitel 2.2.3) und Empfehlungen das Evidenzlevel der zugrunde liegenden Studien sowie bei Empfehlungen zusätzlich die Stärke der Empfehlung (Empfehlungsgrad) ausgewiesen. Hinsichtlich der Stärke der Empfehlung werden in dieser Leitlinie 3 Empfehlungsgrade unterschieden (siehe $>$ Tab.5), die sich auch in der Formulierung der Empfehlungen jeweils widerspiegeln.

Die Entscheidungskriterien für die Festlegung der Empfehlungsgrade werden im Leitlinienreport zu dieser Leitlinie erläutert.

\subsubsection{Statements}

Als Statements werden Darlegungen oder Erläuterungen von spezifischen Sachverhalten oder Fragestellungen ohne unmittelbare Handlungsaufforderung bezeichnet. Sie werden entsprechend der Vorgehensweise bei den Empfehlungen im Rahmen eines formalen Konsensusverfahrens verabschiedet und können entweder auf Studienergebnissen oder auf Expertenmeinungen beruhen.

\subsubsection{Expertenkonsens (EK)}

Statements/Empfehlungen, für die eine Bearbeitung auf der Grundlage von Expertenkonsens der Leitliniengruppe beschlossen wurde, sind als Expertenkonsens ausgewiesen. Für diese Empfehlungen wurde keine systematische Literaturrecherche durchgeführt (die in den Hintergrundtexten ggf. angeführten Studien wurden von den beteiligten Fachexperten ausgewählt). Bei Empfehlungen, die auf einem Expertenkonsens basieren, werden keine Symbole bzw. Buchstaben verwendet, um die Empfehlungsstärke und die Qualität der Evidenz darzustellen. Die Stärke der Empfehlung ergibt sich dann allein aus der verwendeten Formulierung (soll/sollte/kann) entsprechend der Abstufung in \ Tab. $\mathbf{5}$.

\subsubsection{Klug entscheiden}

Empfehlungen, die mit „Klug entscheiden“ gekennzeichnet sind, wurden für die „Klug entscheiden“-Initiative der Deutschen Gesellschaft für Innere Medizin ausgewählt. Diese Empfehlungen sollen als konkrete Hilfestellung bei der Indikationsstellung zu diagnostischen und therapeutischen Maßnahmen dienen, um eine Unter- bzw. Überversorgung zu vermeiden. Weitere Informationen finden Sie unter https://www.klug-entscheiden.com/.

\subsubsection{Unabhängigkeit und Darlegung möglicher Interessenkonflikte}

Die Deutsche Krebshilfe stellte die finanziellen Mittel über das Leitlinienprogramm Onkologie (OL) zur Verfügung. Diese Mittel wurden eingesetzt für Personalkosten, Büromaterial, Literaturbeschaffung und die Konsensuskonferenzen (Raummieten, Technik, Verpflegung, Moderatorenhonorare, Reisekosten der Teilnehmer). Die Erarbeitung der Leitlinie erfolgte in redaktioneller Unabhängigkeit von der finanzierenden Organisation. Alle Mitglieder legten während des Leitlinienprozesses mittels des AWMF-Formblatts eine schriftliche Erklärung zu eventuell bestehenden Interessenkonflikten (zu Beginn, Aktualisierung vor der Konsenskon- 


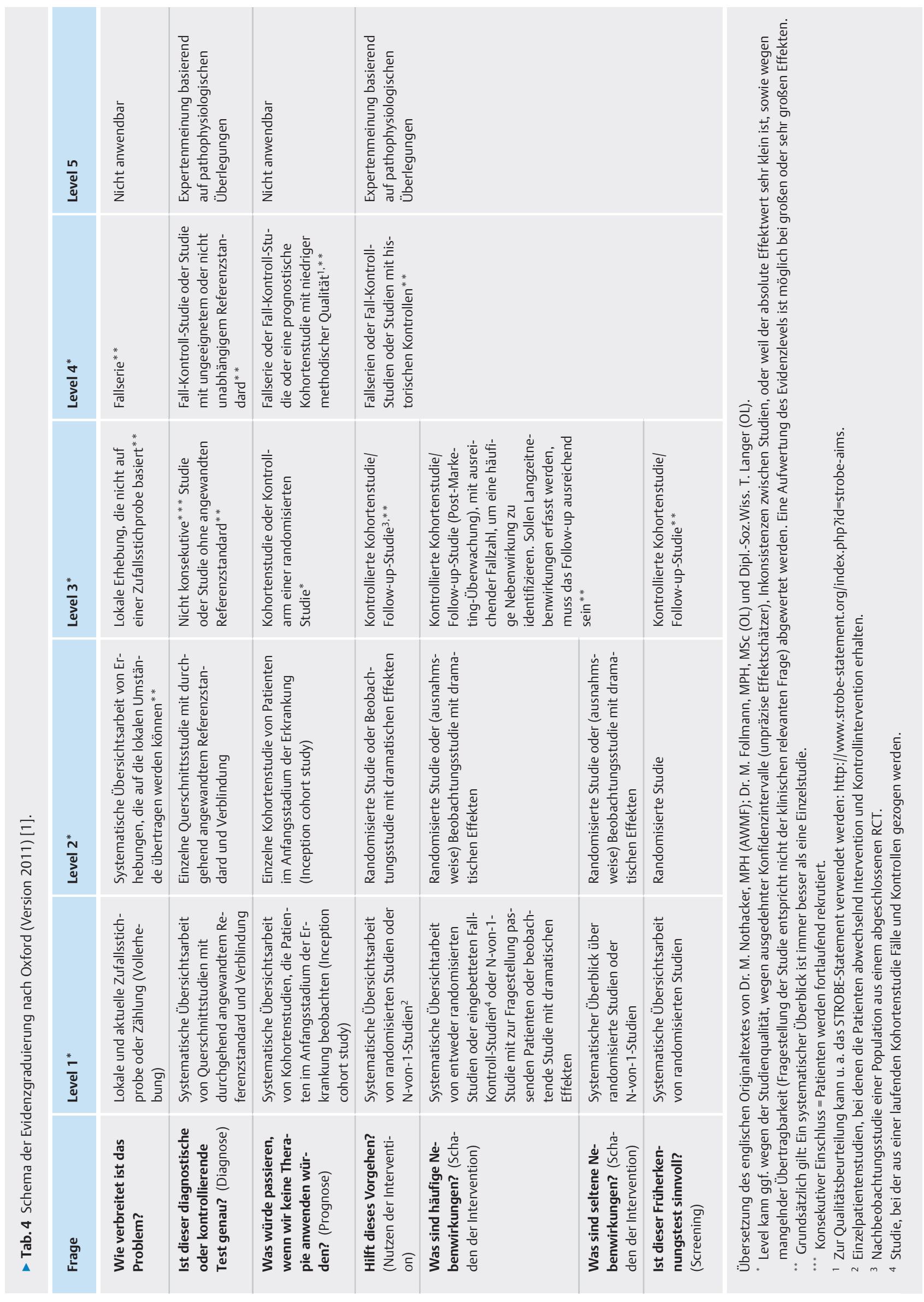


ferenz) vor. Die Interessenerklärungen sind im Leitlinienreport zu dieser Leitlinie (https://www.leitlinienprogramm-onkologie.de/ leitlinien/hepatozellulaeres-karzinom-hcc/) aufgeführt.

Der Umgang mit Interessenkonflikten wurde analog den Vorgaben der AWMF gemeinsam in unserem Steuergruppentreffen vom 18.07.2019 konsentiert. Zusätzlich wurde dieses Vorgehen mit Vertretern der DKG und der AWMF besprochen. Dabei wurde folgendes Vorgehen festgehalten:

- Es wurden alle direkten finanziellen und indirekten sekundären Interessen der letzten 3 Jahre im Formular der Interessenkonflikte angegeben. Unmittelbar vor den beiden Konsensuskonferenzen erfolgte eine Aktualisierung der Erklärung.

- Entscheidend für die Bewertung war der thematische Bezug zur Leitlinie.

- Die Erklärungen zu den Interessenkonflikten werden im Leitlinienreport aufgeführt.

Umgang mit direkten finanziellen Interessenkonflikten:

- Es wurde bei finanziellen Vergütungen durch Ad-Board, Beratertätigkeit, Vortragstätigkeit und Industriedrittmitteln in verantwortlicher Position ein finanzieller Rahmen von $5000 € /$ Jahr und Firma festgesetzt. Oberhalb dieser Grenze kam es zur Feststellung eines moderaten Interessenkonflikts.

- Wurde ein moderater Interessenkonflikt festgestellt, enthielt sich der Mandatsträger bei einzelnen Fragen oder Themenbereichen, je nach festgestelltem Interessenkonflikt.

- Im Hinblick auf die AG-Leiter wurde darauf geachtet, dass einem Mitglied der Steuergruppe mit einem moderateren Interessenkonflikt ein weiteres Mitglied der Steuergruppe mit keinem oder lediglich geringem Interessenkonflikt zur Seite gestellt wurde.

- Bei 2 Mandatsträgern wurden Patente festgestellt. Diese haben jedoch werder einen thematischen Bezug zur Leitlinie, noch sind diese kommerzialisiert. Es erfolgte daher kein Ausschluss von der Leitlinienarbeit.

- Ein Aktienbesitz lag bei keinem Mandatsträger vor.

Umgang mit indirekten sekundären Interessenkonflikten:

- Mitgliedschaften in Fachgesellschaften und Beteiligung an Fortbildungen und Ausbildungsinstituten wurden nicht als Interessenkonflikt für diese Leitlinie bewertet, da diese ein essenzieller Teil der wissenschaftlichen und klinischen Arbeit sind.

- Ebenso wurde der Schwerpunkt der wissenschaftlichen und klinischen Tätigkeit in diesem Feld erwartet, um eine wissenschaftliche und praktikable Leitlinie zu erstellen.

- Eine persönliche Beziehung (Partner oder Verwandter 1. Grades) zu einem Vertretungsberechtigten eines Unternehmens der Gesundheitswirtschaft lag bei keinem Mandatsträger vor.
- Tab. 5 Schema der Empfehlungsgraduierung.

\begin{tabular}{|l|l|l|}
\hline $\begin{array}{l}\text { Empfeh- } \\
\text { lungsgrad }\end{array}$ & Beschreibung & Ausdrucksweise \\
\hline $\mathbf{A}$ & Starke Empfehlung & soll \\
\hline $\mathbf{B}$ & Empfehlung & sollte \\
\hline $\mathbf{0}$ & Empfehlung offen & kann \\
\hline
\end{tabular}

- Tab. 6 Konsensstärke

\begin{tabular}{|l|l|}
\hline Konsensstärke & Prozentuale Zustimmung \\
\hline Starker Konsens & $>95 \%$ der Stimmberechtigten \\
\hline Konsens & $>75-95 \%$ der Stimmberechtigten \\
\hline Mehrheitliche Zustimmung & $50-75 \%$ der Stimmberechtigten \\
\hline Dissens & $<50 \%$ der Stimmberechtigten \\
\hline
\end{tabular}

Der Vorschlag der Steuergruppe zum Management wurde zu Beginn der Konsenskonferenz diskutiert und umgesetzt.

Als protektive Faktoren gegen eine Verzerrung durch Interessenkonflikte können die systematische Evidenzaufbereitung, die pluralistische Zusammensetzung der Leitliniengruppe, die neutrale Moderation, die Diskussion der Bewertung der Interessen und des Umgangs mit Interessenkonflikten zu Beginn der Konsenskonferenz sowie die öffentliche Konsultation gewertet werden.

An dieser Stelle möchten wir allen Mitarbeitern für ihre ausschließlich ehrenamtliche Mitarbeit an dem Projekt danken.

\section{Diagnostik und Therapie des Hepatozellulären Karzinoms}

Die S3-Leitlinie Diagnostik und Therapie des Hepatozellulären Karzinoms und biliärer Karzinome umfasst 2 Tumorentitäten. Zur Vereinfachung sind die Tumorentitäten hier getrennt veröffentlicht. An dieser Stelle verweisen wir daher auf die S3-Leitlinie Diagnostik und Therapie des Hepatozellulären Karzinoms. 


\section{Diagnostik und Therapie der biliären Karzinome}

\subsection{Risikofaktoren, Prävention und Früherkennung}

\subsubsection{Risikofaktoren}

\begin{tabular}{|c|c|c|}
\hline 4.1) & Evidenzbasiertes Statement & Neu 2021 \\
\hline $\begin{array}{l}\text { Level of } \\
\text { Evidence } \\
\mathbf{2 - 3}\end{array}$ & \multicolumn{2}{|c|}{$\begin{array}{l}\text { Risikofaktoren für die Entwicklung eines intra- oder } \\
\text { extrahepatischen Cholangiokarzinoms sind: } \\
\text { - Adipositas } \\
\text { - Alkoholabusus } \\
\text { - Choledochus-Zysten } \\
\text { - Cholelithiasis } \\
\text { - Chronisch bakterielle Cholangitis } \\
\text { - Chronisch entzündliche Darmerkrankungen } \\
\text { - Chronische Hepatitis-B-Virusinfektion } \\
\text { - Chronische Hepatitis-C-Virusinfektion } \\
\text { - Diabetes mellitus } \\
\text { - Leberegel } \\
\text { - Leberzirrhose } \\
\text { - Nichtalkolische Fettlebererkrankung } \\
\text { - Parasitäre Cholangitiden } \\
\text { - Primär sklerosierende Cholangitis } \\
\text { - Rauchen } \\
\text { - Rezidivierende pyogene Cholangitiden }\end{array}$} \\
\hline & \multicolumn{2}{|c|}{$\begin{array}{l}\text { Primärrecherche: Atchison } 2011 \text { [3]; de Valle } 2012 \\
\text { [4]; El-Serag } 2009 \text { [5]; Huang } 2017 \text { [6]; jing } 2012 \\
\text { [7]; Palmer } 2012 \text { [8]; Wongjarupong } 2017 \text { [9] }\end{array}$} \\
\hline & \multicolumn{2}{|l|}{ starker Konsens } \\
\hline 4.2) & Evidenzbasiertes Statement & Neu 2021 \\
\hline $\begin{array}{l}\text { Level of } \\
\text { Evidence } \\
\mathbf{2 - 3}\end{array}$ & \multicolumn{2}{|c|}{$\begin{array}{l}\text { Risikofaktoren für die Entwicklung eines Gallenbla- } \\
\text { senkarzinoms sind: } \\
\text { - Anatomische Anomalien der intra- und extrahe- } \\
\text { patischen Gallenwege } \\
\text { - Cholelithiasis } \\
\text { - Chronisch bakterielle und parasitäre Cholangitis } \\
\text { - Diabetes mellitus } \\
\text { - Gallenblasenpolypen } \\
\text { - Porzellangallenblase } \\
\text { - Primär sklerosierende Cholangitis }\end{array}$} \\
\hline & \multicolumn{2}{|c|}{$\begin{array}{l}\text { Primärrecherche: Atchison } 2011 \text { [3]; Jing } 2012 \text { [7]; } \\
\text { Palmer } 2012 \text { [8]; de Valle } 2012 \text { [4]; Park } 2009 \text { [10]; } \\
\text { Nagaraja } 2014 \text { [11]; }\end{array}$} \\
\hline & \multicolumn{2}{|l|}{ starker Konsens } \\
\hline
\end{tabular}

Cholangiokarzinome (CCA, synonym maligne biliäre Tumoren) sind eine heterogene Gruppe epithelialer Neoplasien, die meistens eine cholangiozytentypische Differenzierung aufweisen [12]. Je nach anatomischer Lokalisation des Primärtumors werden intrahepatische (iCCA) von extrahepatischen (eCCA) CCA und vom Gallenblasenkarzinom unterschieden. Extrahepatische Tumoren werden weiter in perihiläre (synonym Klatskin-Tumor, pCCA) und distale CCA (dCCA) aufgeteilt. Diese Unterscheidung ist aufgrund unterschiedlicher Risikofaktoren, Unterschieden in
Bezug auf molekulare und klinische Charakteristika und unterschiedlicher Therapieansätze relevant [13, 14].

In Deutschland wurden im Jahr 2016 etwa 5290 Menschen mit einem eCCA (etwa $68 \%$; darunter etwa $11 \%$ pCCA) oder einem Gallenblasenkarzinom (etwa $32 \%$ ) diagnostiziert. Hinzu kommen etwa 2000 Patienten mit einem iCCA, die aufgrund der ICD-Kodierung als primäre maligne Lebertumoren erfasst werden. Somit liegt die Gesamtinzidenz in Deutschland bei mehr als 7000 Neuerkrankungen/Jahr (http://krebsdaten.de). Die Inzidenz in Deutschland ist in den letzten 20 Jahren aufgrund der Zunahme des iCCA angestiegen. Mit zunehmendem Lebensalter steigt das Risiko kontinuierlich an. In den letzten 20 Jahren ist die Inzidenz des CCA bei Frauen, insbesondere aufgrund der sinkenden Gallenblasenkarzinom-Inzidenz, leicht rückläufig.

Die Inzidenz der verschiedenen anatomischen Tumorlokalisationen variiert weltweit erheblich, vermutlich aufgrund unterschiedlicher Prävalenzen von Risikofaktoren [15]. In Südostasien ist die CCA-Inzidenz höher als in anderen Ländern. Ein wichtiger Risikofaktor dort sind parasitäre Infektionen mit Leberegeln wie Opisthorchis viverrini oder Clonorchis sinensis, die zu chronischen Cholangitiden führen [16, 17]. Infektionen mit Opisthorchis viverrini oder Clonorchis sinensis sind endemisch in Südostasien und sind mutmaßlich jährlich für mehr als 7000 CCA-Neuerkrankungen in dieser Region verantwortlich [18]. In westlichen Ländern spielen Leberegel-Infektionen als CCA-Risikofaktor keine nennenswerte Rolle. Weitere etablierte Risikofaktoren mit deutlich höherer Inzidenz in Südostasien sind eine Cholelithiasis, Choledochus-Zysten, ein Caroli-Syndrom und rezidivierende pyogene Cholangitiden [15, 19]. Kongenitale Anomalien, z. B. Choledochuszysten oder ein Caroli-Syndrom, weisen ein hohes CCA-Risiko mit einer Prävalenz von bis zu $11 \%$ auf $[20,21]$.

Die primäre sklerosierende Cholangitis (PSC) ist in westlichen Ländern ein relevanter Risikofaktor sowohl für intra- und extrahepatische Cholangiokarzinome als auch für Gallenblasenkarzinome. Das kumulative 10-Jahresrisiko für ein Cholangiokarzinom bei PSC liegt bei $9 \%$ und ist damit deutlich höher als in der Allgemeinbevölkerung [22]. Ob bei PSC-Patienten Alkoholkonsum und Nikotin weitere Ko-Risikofaktoren darstellen, ist weiter unklar. Ebenso bleibt unklar, ob eine chronisch entzündliche Darmerkrankung ohne Vorhandensein einer PSC einen relevanten Risikofaktor darstellt [23]. Eine Leberzirrhose, eine chronische Hepatitis-Coder Hepatitis-B-Virusinfektion, Alkoholkonsum und Diabetes sind in westlichen Ländern wichtige Risikofaktoren für die Entwicklung eines iCCA $[8,15,24]$. Insbesondere ist das Risiko für das iCCA bei Diabetes und/oder Adipositas erhöht [25]. Bei nichtalkoholischer Fettlebererkrankung (NAFLD) ist das iCCA-Risiko leicht erhöht [9]. Ebenso weisen Raucher ein gering erhöhtes Risiko für die Entwicklung eines intra- und extrahepatischen CCAs, jedoch nicht für ein Gallenblasenkarzinom auf [24]. Weitere etablierte Risikofaktoren sind eine Cholelithiasis und biliäre Zysten. Das höchste Risiko sowohl für die Entwicklung eines iCCA als auch eines eCCA weisen Patienten mit Choledochuszysten auf (relatives Risiko 26,7 bzw. 34,9) [26].

Frauen erkranken häufiger als Männer an einem Gallenblasenkarzinom. Der wichtigste Risikofaktor für die Entwicklung eines Gallenblasenkarzinoms jedoch sind Gallensteine. Etwa 70-90\% aller Patienten mit einem Gallenblasenkarzinom weisen eine Cho- 
lelithiasis auf. Insbesondere findet sich eine hohe Gallblasenkarzinom-Inzidenz in der indigenen Bevölkerung Nord- und Lateinamerikas und Neuseelands. Weitere wichtige Risikofaktoren für die Entwicklung eines Gallenblasenkarzinoms sind ein höheres Alter, Adipositas, eine familiäre Häufung und Gallenblasenpolypen [27, 28]. Gallenblasenpolypen können eine Wachstumstendenz aufweisen und so schließlich entarten. Genaue Daten dazu sind jedoch aufgrund des langsamen Wachstums - oftmals über Dekaden - schwer zu interpretieren [28]. In einer großen Kohortenstudie mit Langzeitverlauf zeigte sich eine Detektionsrate neoplastischer Polypen (Adenom oder Gallenblasenkarzinom) von 1,7\% nach einem Jahr, von 2,8\% nach 5 Jahren und von $4 \%$ nach 8 Jahren nach erstmaliger Diagnosestellung eines Gallenblasenpolypen [10]. Die Prävalenz von Gallenblasenpolypen in Deutschland beträgt bis zu $6 \%$. Die Adenom-Prävalenz liegt unter $5 \%$ [29]. Als Risikofaktoren für einen neoplastischen Polypen zeigten sich das gleichzeitige Vorhandensein einer Cholelithiasis und die Polypengröße. Polypen mit $\geq 10 \mathrm{~mm}$ weisen ein 24 -fach erhöhtes Risiko für eine Malignität auf [10].

Ein weiterer Risikofaktor für ein Gallenblasenkarzinom ist eine chronische Entzündung, verursacht durch Salmonella typhi bzw. parathyphi oder Helicobacter bilis [11]. Eine Besiedelung der Gallenblase mit Salmonella typhi bei Dauerausscheidern ist mit einem 12-fach erhöhten Risiko für die Entwicklung eines Gallenblasenkarzinoms assoziiert [28].

Die als klassische Präkanzerose angesehene Kalzifizierung der Gallenblasenwand, die sogenannte Porzellangallenblase, wird in der jüngeren Literatur als Risikofaktor für ein Gallenblasenkarzinom differenzierter betrachtet [30, 31]. Das relative Risiko für ein Gallenblasenkarzinom liegt bei 4,6 [32] und ist niedriger als in älteren Arbeiten angegeben [33].

\subsubsection{Prophylaktische und therapeutische Maßnahmen zur Reduktion des Risikos der Entstehung von biliären Karzinomen}

\begin{tabular}{|c|c|c|}
\hline 4.3) & Konsensbasierte Empfehlung & Neu 2021 \\
\hline EK & \multicolumn{2}{|c|}{$\begin{array}{l}\text { Gallenblasenpolypen bei Patienten mit PSC sollten } \\
\text { regelmäßig sonografisch überwacht werden. In allen } \\
\text { Fällen sollte die Indikation zur Cholezystektomie disku- } \\
\text { tiert werden, bei Polypen über } 8 \text { mm oder Größenpro- } \\
\text { gredienz sollte aufgrund des erhöhten Karzinomrisikos } \\
\text { unter Berücksichtigung der Leberfunktion eine Chole- } \\
\text { zystektomie erfolgen. }\end{array}$} \\
\hline & starker Konsens & \\
\hline
\end{tabular}

Für die asymptomatische Bevölkerung ist eine CCA-Früherkennung aufgrund der niedrigen Inzidenz nicht sinnvoll.

Gallenblasenpolypen treten bei bis zu 13,7\% der PSC-Patienten auf, das Risiko für ein Gallenblasenkarzinom ist hoch und steigt mit zunehmender Größe. Die Empfehlung zur Cholezystektomie ab einer Größe von $8 \mathrm{~mm}$ basiert auf einer retrospektiven Kohortenstudie mit 57 PSC-Patienten [34]. Eine frühzeitige Cholezystektomie kann auch bei kleineren Polypen diskutiert werden. Für detaillierte Empfehlungen zum Management von Patienten mit
PSC wird auf die aktuelle S2-Leitlinie „Autoimmune Lebererkrankungen“ verwiesen [35].

\begin{tabular}{|l|l|l|}
\hline $\mathbf{4 . 4 )}$ & Konsensbasierte Empfehlung & Neu 2021 \\
\hline EK & $\begin{array}{l}\text { Patienten mit Gallenblasenpolypen } \geq 10 \text { mm sollte } \\
\text { unabhängig von der Symptomatik eine Cholezystekto- } \\
\text { mie angeboten werden. }\end{array}$ \\
\hline & starker Konsens \\
\hline
\end{tabular}

Die Indikationsstellung zur Therapie bei Cholezystolithiasis und Gallenblasenpolypen erfolgt gemäß der aktuellen S3-Leitlinie zur Prävention, Diagnostik und Behandlung von Gallensteinen [33]. Aufgrund des deutlich erhöhten Malignitätsrisikos bei Polypen $\geq 1 \mathrm{~cm}$ Durchmesser bei gleichzeitig vorhandener Cholezystolithiasis als weiterem Risikofaktor wird eine Cholezystektomie unabhängig von der Symptomatik empfohlen. Bei fehlender Indikation für eine Cholezystektomie wird ein individuelles Vorgehen basierend auf dem Vorhandensein von Risikofaktoren für neoplastische Gallenblasenpolypen (Alter > 50 Jahre, bekannte PSC, Zugehörigkeit zu einer indigenen Population oder Vorhandensein eines sessilen Polypen) in Analogie zu einem Delphi-Methode-basierten Expertenkonsens empfohlen [36].

\begin{tabular}{|c|c|c|}
\hline 4.5) & Konsensbasierte Empfehlung & Neu 2021 \\
\hline EK & \multicolumn{2}{|c|}{$\begin{array}{l}\text { Bei Patienten ohne Risikofaktoren* für ein Gallenblasen- } \\
\text { karzinom mit Nachweis eines Gallenblasenpolypen von } \\
<9 \text { mm sollte eine sonografische Kontrolle in } 6 \text { Monaten } \\
\text { (Polyp 6-9 mm) bzw. } 12 \text { Monaten (Polyp <6 mm) } \\
\text { erfolgen. } \\
\text { * Risikofaktoren für neoplastische Polypen: Alter } \\
\text { > } 50 \text { Jahre, bekannte PSC, Zugehörigkeit zu einer } \\
\text { indigenen Population oder Vorhandensein eines } \\
\text { sessilen Polypen [36] }\end{array}$} \\
\hline & Konsens & \\
\hline
\end{tabular}

\subsubsection{Früherkennung}

\begin{tabular}{|l|l|}
\hline 4.6) & Konsensbasierte Empfehlung \\
\hline EK & $\begin{array}{l}\text { Bei Patienten mit PSC sollte halbjährlich eine Bildgebung } \\
\text { im Rahmen der Cholangiokarzinom-Früherkennung } \\
\text { durchgeführt werden. }\end{array}$ \\
\hline & starker Konsens \\
\hline
\end{tabular}

Im Gegensatz dazu besteht aufgrund des deutlich erhöhten CCA-Risikos bei Patienten mit einer PSC ein großer Bedarf für eine effektive Früherkennung. Daten, die einen Überlebensvorteil einer CCA-Früherkennung bei Patienten mit PSC zeigen, fehlen jedoch [35]. In vielen Zentren erfolgen heute bei Patienten mit gesicherter PSC eine CCA-Früherkennung mittels Magnetresonanztomografie (MRT) und Magnetresonanzcholangiopankreati- 
kografie (MRCP) in 6- bis12-monatlichem Abstand und zusätzlich die serielle Bestimmung des Tumormarkers CA19-9 [37]. Für eine MRT/MRCP-basierte Früherkennung spricht eine hohe Sensitivität von $89 \%$ bei einer Spezifität von $75 \%$. Die Sensitivität des Ultraschalls ist niedriger, aufgrund der Verfügbarkeit, der niedrigeren Kosten und der großen Akzeptanz findet die Sonografie jedoch breite Anwendung bei der Überwachung von PSC-Patienten [38].

Im Rahmen der Konsensuskonferenz wurde festgehalten, dass aufgrund der höheren Sensitivität eine Abdomensonografie im Wechsel mit einem nativen MRT inklusive MRCP erfolgen sollte. Dabei soll sowohl die Leber als auch die Gallenblase mitbeurteilt werden.

\subsection{Histopathologische und molekulare Diagnostik}

\subsubsection{Typisierung und Staging von biliären Karzinomen}

\begin{tabular}{|l|l|l|}
\hline 4.7) & Konsensbasierte Empfehlung & Neu 2021 \\
\hline EK & $\begin{array}{l}\text { Vor oder im Rahmen einer Tumortherapie sollen Tumoren } \\
\text { der Gallenwege und Gallenblase histologisch oder ggf. } \\
\text { zytologisch gesichert werden }\end{array}$ \\
\hline & Konsens \\
\hline
\end{tabular}

Aufgrund des Fehlens beweisender positiver bildgebender diagnostischer Kriterien ist die histologische Sicherung der intrahepatischen Cholangiokarzinome grundsätzlich erforderlich (siehe - Abb. 1 Diagnosealgorithmus eines Patienten mit einem Verdacht auf ein Cholangiokarzinom S. 200). Dies ist besonders relevant, da der Mehrzahl der Cholangiokarzinome keine spezifische chronische Vorerkrankung zugrunde liegt, sodass deren positive prädiktive Aussagekraft, anders als z. B. beim HCC, nicht mit in Betracht gezogen werden kann. Dennoch treten intrahepatische Cholangiokarzinome auch bei den zum HCC prädisponierenden chronischen Lebererkrankungen und der Zirrhose vermehrt auf, sodass die Möglichkeit eines intrahepatischen Cholangiokarzinoms, auch bei bildgebendem Verdacht auf ein HCC, differenzialdiagnostisch zu berücksichtigen ist. Grund dafür sind v. a. Fälle intrahepatischer Cholangiokarzinome, die die bildgebenden Kriterien eines HCC zeigen können. Umgekehrt können bei einzelnen HCCs, insbesondere vom sklerotischen Subtyp, die bildgebenden Kriterien eines HCC fehlen und zum Verdacht auf ein iCCA führen.

Bei Tumoren der extrahepatischen Gallenwege kann die Sicherung eines invasiven Karzinoms in Abgrenzung von entzündlich bedingten Veränderungen und nichtinvasiven prämalignen Veränderungen problematisch sein; dies gilt besonders auch für die Abklärung dominanter Stenosen bei der PSC. Die Sensitivität histologischer und zytologischer Verfahren in der Detektion invasiver Karzinome der distalen Gallenwege ist derzeit noch begrenzt und überschreitet auch im kombinierten Einsatz gemäß der meisten Untersuchungen nicht 60-70\% [39, 40]. Zusätzliche Verfahren wie FISH-Analysen und auch molekulare Analysen am Gallesekret mögen in Einzelfällen unterstützende Informationen liefern, können aber weder die histologische oder zytologische Diagnostik ersetzen, noch kann mangels entsprechender Validierung ihr diagnostischer Einsatz derzeit generell empfohlen werden.
In Fällen einer anstehenden Resektion bei hochgradigem Verdacht auf ein Karzinom der extrahepatischen Gallenwege kann angesichts der eingeschränkten Sensitivität zytologischer und bioptischer Nachweise auf eine präoperative bioptische Sicherung zugunsten einer umfassenden Aufarbeitung des Resektionspräparats verzichtet werden, wenn die Abwägung der möglichen Vorund Nachteile keine Verbesserung für den Patienten erwarten lässt. Die Entscheidungsfindung sollte durch ein interdisziplinäres Tumorboard abgesichert sein.

Bei nichtoperablen intra- und extrahepatischen Cholangiokarzinomen und Karzinomen der Gallenblase soll vor Einleitung einer Therapie eine histologische Sicherung erfolgen, wobei das dabei gewonnene Gewebe in der Regel zusätzlich auch für eventuelle weiterführende, z. B. molekularpathologische Untersuchungen ausreichen sollte und dafür bei Bedarf einzusetzen ist.

\begin{tabular}{|c|c|c|}
\hline 4.8) & Konsensbasierte Empfehlung & Neu 2021 \\
\hline EK & \multicolumn{2}{|c|}{$\begin{array}{l}\text { Die Typisierung der Karzinome der Gallenwege und der } \\
\text { Gallenblase soll nach der anatomischen Lokalisation } \\
\text { (intrahepatisch, perihilär, distale Gallenwege, Gallenblase) } \\
\text { und gemäß der histologischen Differenzierung nach der } \\
\text { aktuellen WHO-Klassifikation erfolgen. Bei intrahepati- } \\
\text { schen Cholangiokarzinomen sollte eine Unterscheidung } \\
\text { von ,Small duct‘- und ,Large duct'-Typ erfolgen. }\end{array}$} \\
\hline & starker Konsens & \\
\hline
\end{tabular}

Klinisch, bildgebend und beim Staging werden die Karzinome der Gallenwege und der Gallenblase gemäß der anatomischen Lokalisation des Ausgangstumors beschrieben [41, 42]. Karzinome des distalen extrahepatischen Gallengangs liegen distal der Mündung des D. cysticus in den D. choledochus. Karzinome der Gallenblase umfassen die Tumoren der Gallenblase und des D. cysticus. Karzinome der perihilären Gallenwege umfassen Tumoren des D. hepaticus dexter und sinister sowie des D. hepaticus communis. Intrahepatische Cholangiokarzinome (iCCA) haben ihren Ausgang von den intrahepatischen Gallenwegen proximal des D. hepaticus dexter bzw. sinister.

Gemäß der WHO-Klassifikation (5. Auflage) ist bei iCCA ein phänotypisch den kleinen Gallengängen ähnlicher ,Small duct'Typ von einem den Karzinomen der extrahepatischen Gallengänge vergleichbaren ,Large duct'-Typ zu unterscheiden [42]. Beide Tumortypen sind ätiologisch, molekular, histologisch, bildgebend und klinisch in ihrer typischen Ausprägung verschieden, sodass ihre Unterscheidung von prognostischer und zunehmend auch therapeutischer Bedeutung ist ( $\triangleright$ Tab. 7: Typische Merkmale der CCA-Subtypen [42-45]). Diagnostisch können beide Tumortypen histologisch und ggf. immunhistologisch oder aufgrund ihrer molekularen Eigenschaften unterschieden werden.

Differenzialdiagnostisch müssen iCCAs vor allem von Metastasen extrahepatischer Karzinome in der Leber unterschieden werden. Metastasen sind in der Summe erheblich häufiger als iCCAs, sodass die möglichst sichere Unterscheidung wichtig ist. Vor allem Metastasen eines Pankreaskarzinoms, aber auch anderer Karzinome (besonders Lunge, Magen, Mamma) und seltener Metastasen neuroendokriner Neoplasien sind zu berücksichtigen. 


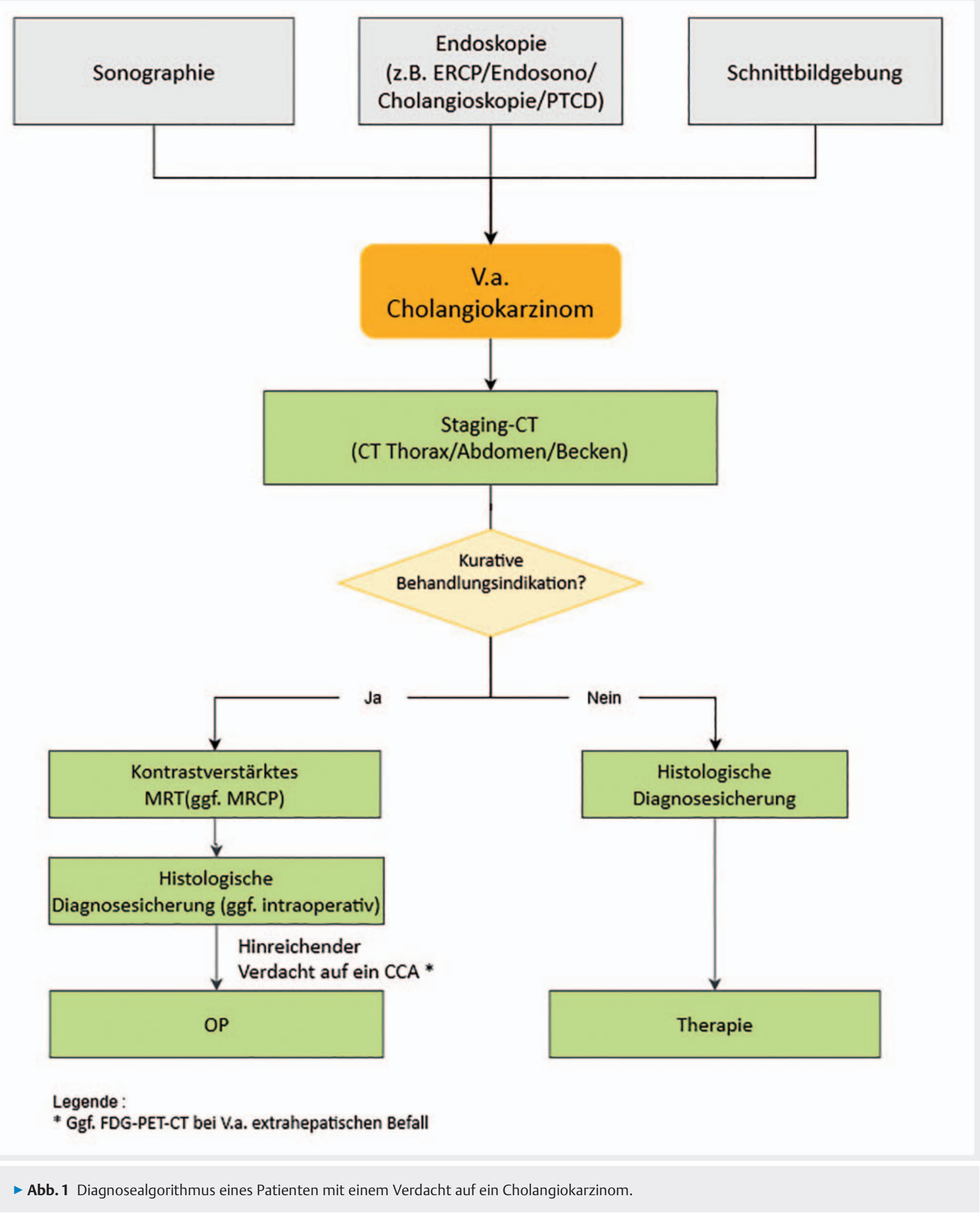


- Tab. 7 Typische Merkmale der CCA-Subtypen [42-45].

\begin{tabular}{|l|l|l|}
\hline Kriterien & Small-duct Type iCCA & Large-duct Type iCCA, distales CCA \\
\hline Prädisponierende Erkrankungen & $\begin{array}{l}\text { Chronische Hepatitis B/C, NASH, andere } \\
\text { chronische Lebererkrankungen (Zirrhose) }\end{array}$ & $\begin{array}{l}\text { PSC, biliäre Helminthosen } \\
\text { (C. sinensis, O. viverrini), Konkremente }\end{array}$ \\
\hline Prämaligne Läsionen & unbekannt & BillN, IPNB, MCN \\
\hline Makroskopie & Primär knotenbildend (,mass forming') & Primär periduktal infiltrierend \\
\hline Histologie & $\begin{array}{l}\text { Zellreicher, weniger Stroma, kein Muzin, } \\
\text { kohärenter wachsend }\end{array}$ & $\begin{array}{l}\text { Tumorzellärmer, stromareich, (extrazelluläre) } \\
\text { Muzinbildung; verstreutes Wachstum }\end{array}$ \\
\hline Molekulare Veränderungen & FGFR2-TL, IDH1/2, BAP1, p53, KRAS ARID1A & KRAS, p53, ARID1B, SMAD4 \\
\hline Systemtherapeutische Zielstrukturen & $\begin{array}{l}\text { Ergiebig; v. a. IDH1/2-Mut; FGFR-2-TLs, andere TLs } \\
\text { (incl. NTRK), BRAF-Mut; MSIhigh }\end{array}$ & Weniger; BRCA-1/2-Mut; Her-2-Amp; MSIhigh \\
\hline
\end{tabular}

Eine definitive Unterscheidung einer Lebermetastase eines exokrinen Pankreaskarzinoms (oder eines Karzinoms der extrahepatischen Gallenwege oder Gallenblase) von einem iCCA (v. a. „Large duct“-Typ) ist an der Biopsie in den meisten Fällen weder histologisch noch immunhistologisch sicher möglich, sodass die Diagnose im Kontext des klinisch-bildgebenden Befundes zu stellen ist.

Ferner sollten bei gesicherter intrahepatischer Tumorlokalisation seltenere gemischte Tumorformen (gemischt neuroendokrine/nicht neuroendokrine Neoplasien (MINEN) und kombinierte Hepato-Cholangiokarzinome (c(ombined) HCC/CCA)) vom iCCA unterschieden werden. Das früher dem cHCC/CCA zugeschlagene Cholangiokarzinom wird aufgrund neuer molekularer Befunde [46] heute als Sonderform des iCCA klassifiziert. Solide wachsende iCCA sind insbesondere vom sklerosierten Subtyp des HCC zu unterscheiden; dafür sind in der Regel zusätzliche immunhistologische Untersuchungen erforderlich (s. u.). Diese Unterscheidung ist auch deshalb wichtig, da HCCs vom sklerosierten Subtyp auch bildgebend meist nicht als HCCs erkannt werden. Seltener stellen die meist als Zufallsbefunde bei Laparotomien erfassten Gallengangsadenome eine Differenzialdiagnose zu kleinen hochdifferenzierten iCCA dar.

Karzinommetastasen in die distalen Gallenwege oder die Gallenblase sind extrem selten und treten nicht isoliert und nur im terminalen Krankheitsstadium auf, sodass sich diagnostisch bei gesicherter Lokalisation diese differenzialdiagnostische Frage nicht stellt. Dann müssen die Karzinome ggf. von seltenen neuroendokrinen Neoplasien, mesenchymalen und neuroektodermalen Tumoren unterschieden werden [47].

\subsubsection{Zytologische und histopathologische} Untersuchungen zur Diagnostik eines CCA oder eines Gallenblasenkarzinoms

\begin{tabular}{|l|l|l|}
\hline $\mathbf{4 . 9 )}$ & Konsensbasierte Empfehlung & Neu 2021 \\
\hline EK & $\begin{array}{l}\text { Die Bearbeitung und Befundung eines Resektats soll die } \\
\text { Ausdehnung des Tumors (Staging) gemäß der aktuellen } \\
\text { TNM-Klassifikation, seinen Typ (Typing) und Differenzie- } \\
\text { rungsgrad (Grading) und den Status des Resektatrandes } \\
\text { (R-Klassifikation) sowie bei intrahepatischen Cholangio- } \\
\text { karzinomen den Status der nichttumorösen Leber ermit- } \\
\text { teln. Bei Präparaten mit prämalignen Läsionen soll durch } \\
\text { genaue Aufarbeitung ein möglicher Übergang in ein inva- } \\
\text { sives Karzinom abgeklärt werden. }\end{array}$ \\
\hline & starker Konsens \\
\hline
\end{tabular}

Das pathohistologische Staging eines Karzinoms der Gallenwege hat unabhängige prognostische Bedeutung und erfolgt gemäß der geltenden TNM-Klassifikation (derzeit 8. Auflage), wobei für alle 4 anatomischen Lokalisationen (intrahepatisch, perihilär, distal, Gallenblase) eigene TNM-Klassifikationen existieren [48]. Zusätzlich zu den Hauptkriterien sollten auch die Nebenkriterien Lymphgefäßeinbruch (L), Veneneinbruch (V) und vor allem bei perihilären und distalen Cholangiokarzinomen auch die Nervenscheideninfiltration (Pn) beurteilt werden. Bezüglich des Typings sollte die aktuelle WHO-Klassifikation Berücksichtigung finden [41, 42]. Das Typing hat prognostische und in einem Teil der Fälle (s. z. B. molekulare Diagnostik und gezielte Therapieansätze) prädiktive Bedeutung. Die prognostische Bedeutung des Gradings ist nach bisherigen Daten gering; es existiert kein uniform akzeptiertes, spezifisches Gradingschema für die einzelnen Cholangiokarzinomtypen und -lo- 
kalisationen, sodass das allgemeine UICC-Gradingschema [48], ggf. gemäß den Anweisungen der ADT [49], angewandt werden sollte. Insbesondere extrahepatische Cholangiokarzinome neigen, vergleichbar mit exokrinen Pankreaskarzinomen zu periduktaler, lymphangischer, perineuraler und vereinzelt auch diskontinuierlich erscheinender Ausbreitung, sodass an eine In-sano-Resektion und die entsprechende histologische Aufarbeitung der gesamten Resektionsränder eine hohe Anforderung besteht. Es wird daher wegen der Bedeutung für die Tumorrekurrenz empfohlen, den genauen Abstand zum Resektionsrand (in mm) anzugeben und dabei die ,R0 wide'-Definition (1 mm) zu beachten.

Biliäre intraepitheliale Neoplasien (BilIN), intraduktale papilläre Neoplasien der Gallenwege (IPNB), Muzinös-zystische Neoplasien (MCN) und Adenofibrome sind benigne Läsionen, die ein unterschiedlich hohes, jedoch signifikantes, malignes Entartungspotenzial in ein Cholangiokarzinom aufweisen. Bei Vorliegen einer derartigen prämalignen Läsion ist der Dysplasiegrad (niedrig, hoch) anzugeben und durch eine entsprechend ausreichende makroskopische und histologische Aufarbeitung der Übergang in ein invasives Karzinom auszuschließen.

Auch wenn sich bei der Mehrzahl der Cholangiokarzinome keine prädisponierende Grunderkrankung eruieren lässt, bedingen chronische Lebererkrankungen und die Zirrhose, vergleichbar dem HCC, ein erhöhtes Risiko, auch an einem iCCA zu erkranken. Ferner kann der Status der nichttumorösen Leber ggf. Prognose und Therapie beeinflussen und sollte daher, wenn immer er ausreichend beurteilbar ist, diagnostisch festgehalten werden.

\begin{tabular}{|l|l|l|}
\hline $\mathbf{4 . 1 0 )}$ & Konsensbasierte Empfehlung & Neu 2021 \\
\hline EK & $\begin{array}{l}\text { Die Diagnose eines Cholangiokarzinoms kann bei klarer } \\
\text { Konstellation durch die konventionelle Histologie } \\
\text { gestellt werden. } \\
\text { In unklaren Fällen, insbesondere bei intrahepatischen } \\
\text { Tumoren, soll die Diagnose durch geeignete immunhis- } \\
\text { tologische und oder molekularpathologische Untersu- } \\
\text { chungen abgesichert werden. }\end{array}$ \\
\hline & starker Konsens \\
\hline
\end{tabular}

In der Regel weisen Cholangiokarzinome ein duktales Wachstumsmuster und eine typischerweise ausgeprägte tumorassoziierte Stromareaktion auf, wobei letztere beim iCCA vom „Small-duct“Typ geringer ausgebildet ist. Sowohl beim intra- als auch beim extrahepatischen Cholangiokarzinom sind jedoch seltenere histologische Subtypen bekannt, die ein davon abweichendes histologisches oder zytologisches Erscheinungsbild aufweisen. Die sichere Einordnung dieser Sonderformen als Cholangiokarzinom und ggf. Unterscheidung von Tumoren anderer Primärlokalisation kann zusätzliche Untersuchungen einschließlich des klinisch-bildgebenden Ausschlusses eines extrabiliären Primärtumors erfordern.

Die Immunhistologie kann die Diagnose eines Cholangiokarzinoms unterstützen, wobei es keine beweisende (liniendefinieren- de) immunhistologische Markerkonstellation gibt. Die Positivität für K7, K19 und Ca19-9 legt eine pankreato-biliäre (d. h. eine dem exokrinen Pankreas und den Gallenwegen entsprechende) Differenzierung in der Unterscheidung von Metastasen anderer extrahepatischer Primärtumoren nahe. Ansonsten sollten die entsprechenden immunhistologischen linientypischen Nachweise der infrage kommenden Differenzialdiagnosen (bei HCC z. B. HepPar1, Arginase 1; bei anderen Adenokarzinomen die für sie typischen immunhistologischen Marker) eingesetzt werden. Sollte bei einem iCCA die Unterscheidung eines „Large duct“-Subtyps von einem „Small duct“-Subtyp histologisch nicht mit der gebotenen Sicherheit möglich sein, können Spezialfärbungen (MuzinProduktion), Immunhistologie und in Ausnahmefällen auch die Molekularpathologie eine definitive Zuordnung ermöglichen.

Eine relevante klinische Fragestellung ist das sog. Adenokarzinom-Carcinoma of Unknown Primary (Adeno-CUP) der Leber [47]; darunter versteht man ein für eine Metastasenleber typisches Bild, ohne dass sich ein extrahepatischer Primärtumor definieren lässt. Tatsächlich kann sich neben verschiedenen, nicht detektierten, extrahepatischen Primärtumoren (v. a. Pankreas, Gastrointestinaltrakt, Lunge, Mamma) hinter einem Adeno-CUP auch ein unerkanntes iCCA verbergen. Da CUPs, die sekundär einer definitiven Typisierung zugeführt werden können, wohl auch dank zunehmend erweiterter Therapieoptionen, gegenüber CUPs, die eine StandardChemotherapie erhalten, eine bessere Prognose aufweisen, sollte bei klinischer Relevanz die notwendige histologische, immunhistologische und ggf. molekularpathologische Differenzialdiagnostik durchgeführt werden.

\subsubsection{Molekulare Diagnostik}

\begin{tabular}{|l|l|l|}
\hline $\mathbf{4 . 1 1 )}$ & Konsensbasierte Empfehlung & Neu 2021 \\
\hline EK & $\begin{array}{l}\text { Cholangiokarzinome weisen potenzielle Zielstrukturen } \\
\text { für eine molekular gesteuerte Systemtherapie auf, die im } \\
\text { geeigneten Kontext getestet werden sollten. }\end{array}$ \\
\hline & starker Konsens \\
\hline
\end{tabular}

Karzinome der Gallenwege und der Gallenblase können molekulare Veränderungen aufweisen, die Angriffspunkte für neue, teils zugelassene, teils in Zulassungsstudien befindliche, gezielte Therapeutika darstellen. Art und Häufigkeit der Veränderungen unterscheiden sich erheblich zwischen den verschiedenen Typen, sodass der präzisen morphologischen Typisierung auch Bedeutung für die Ausrichtung einer evtl. molekularen Testung und ggf. daraus abgeleiteten, therapeutischen Zielstruktur zukommt. Insbesondere das iCCA vom „Small duct“-Subtyp zeigt derartige molekulare Veränderungen in besonders hoher Frequenz. Vor allem an spezialisierten Zentren wird zunehmend eine umfassende Testung eingesetzt, um primär therapeutisch angehbare und studiengängige Zielstrukturen zu erfassen. - Tab. $\mathbf{8}$ fasst die wichtigsten Zielstrukturen zusammen. 
- Tab. 8 Molekulare Alterationen beim small duct iCCA.

\begin{tabular}{|l|l|l|}
\hline Molekulare Alteration & Häufigkeit (\%) & Zulassung \\
\hline RAS-Mutation & $10-20$ & \\
\hline TP53-Mutation & $20-30$ & \\
\hline FGFR2-Translokation & $15-30$ & Zulassung \\
\hline IDH1/2 & $10-20$ & $\begin{array}{l}\text { Zulassung außerhalb } \\
\text { der EU }\end{array}$ \\
\hline ARID1A & $5-15$ & \\
\hline BAP1 & $5-15$ & $\begin{array}{l}\text { Zulassung bei anderer } \\
\text { Entität }\end{array}$ \\
\hline BRAF V600E & $3-6$ & $\begin{array}{l}\text { Zulassung bei anderer } \\
\text { Entität }\end{array}$ \\
\hline ERBB2 & $2-3$ & $\begin{array}{l}\text { Zulassung außerhalb } \\
\text { der EU }\end{array}$ \\
\hline MSI-H (MLH1, MSH2, & $1-2$ & Zulassung \\
\hline MSH6, PMS2) & $<1$ & $\begin{array}{l}\text { Zulassung bei anderer } \\
\text { Entität }\end{array}$ \\
\hline NTRK1-3 & $<1$ & \\
\hline NRG1 & & \\
\hline
\end{tabular}

\subsection{Bildgebende und endoskopische Diagnostik}

4.3.1. Bildgebende und/oder endoskopische Untersuchungen zum Staging und zur Diagnosestellung eines biliären Karzinoms

\begin{tabular}{|l|l|l|}
\hline 4.12) & Konsensbasiertes Statement & Neu 2021 \\
\hline EK & $\begin{array}{l}\text { Die Sonografie wird häufig zur initialen Einschätzung } \\
\text { bei V.a. auf ein biliäres Karzinom verwendet. }\end{array}$ \\
\hline & starker Konsens \\
\hline
\end{tabular}

Die abdominelle Sonografie wird meist initial eingesetzt zur Abklärung erhöhter Leberwerte und ist in Endemiegebieten Südostasiens Methode der Wahl zum jährlichen Screening auf biliäre Karzinome [50]. Das intrahepatische CCA ist meist iso- bis hypoechogen, gelegentlich ist zusätzlich oder bei periduktal infiltrierendem Tumorwachstum als alleiniges Merkmal eine umschriebene Gangdilatation stromaufwärts des iCCA darstellbar. In der Kontrastmittelsonografie (CEUS) ist das Hyperenhancement uneinheitlich und abhängig von Tumorzelldichte und Fibrose des Tumors sowie Entzündung und Fibrose in der umgebenden Leber [51, 52]. In einer DEGUM-Multizenterstudie zeigte sich eine in der Tumorperipherie betonte initiale Kontrastmittelanflutung bei $75 \%$ der iCCA, mit früh-portalvenöser Auswaschung vor allem im Tumorzentrum bei 85,8\% und Auswaschung in der Spätphase bei 92,9\% [53]. Das iCCA zeigt im Unterschied zum HCC häufig ein früh (<60 Sekunden) beginnendes, deutlich ausgeprägtes Auswaschen. In älteren Studien war die Pfortaderinfiltration mit hoher
Genauigkeit darstellbar [54]. Die Darstellung von Gallenblasenpolypen gelingt mit dem Ultraschall mit relativ hoher Genauigkeit, die Differenzierung maligner von nicht malignen Polypen wird in einem 2018 publiziertem Cochrane-Review mit einer Sensitivität von 0,79 und einer Spezifität von 0,89 angegeben [55].

Neben der mittels Ultraschalls geäußerten Verdachtsdiagnose auf ein iCCA handelt es sich auch oft um einen Zufallsbefund in aus anderen Gründen durchgeführten bildgebenden Untersuchungen. Die bildgebenden Merkmale der iCCA sind oft suggestiv für die Diagnose, aber nicht definitiv genug, um eine Biopsie überflüssig zu machen (siehe $\mathbf{A b b} \mathbf{1}$ Diagnosealgorithmus eines Patienten mit einem Verdacht auf ein Cholangiokarzinom S. 200).

Ein typisches kontrastverstärktes CT-Protokoll für die Diagnose und das initiale Staging eines Cholangiokarzinoms umfasst eine arterielle (20-30 Sekunden nach der Injektion), eine portalvenöse (60 Sekunden nach der Injektion) und eine Spätphase (mindestens 3 Minuten nach der Injektion) [56-58]. CT-morphologisch ist das typische Erscheinungsbild eine hypodense Leberraumforderung mit unregelmäßigen Rändern in der nativen Phase, einem hypervaskulären Saum in der arteriellen Phase und ein zunehmendes Enhancement in der venösen Phase und den Spätphasen [59]. Mittels CT kann auch der Grad der biliären Obstruktion, der Kapselretraktion oder der hepatischen Atrophie erkannt werden. Die dynamische CT-Untersuchung kann bei der Unterscheidung zwischen iCCA und HCC helfen. Bis zu 81 \% der iCCA sind durch eine progressive Kontrastmittelaufnahme von der arteriellen zur venösen Phase und insbesondere in der Spätphase gekennzeichnet. Dieser Effekt kann auf eine Fibrose zurückzuführen sein, die das intravenöse Kontrastmittel zurückhält. Im Gegensatz dazu ist das HCC durch eine arterielle Hypervaskularisation während der arteriellen Phase und ein Auswaschen in der venösen Phase oder in der Spätphase charakterisiert. Einige kleine iCCA können aber auch eine arterielle Hypervaskularisation aufweisen und können dadurch ein Hepatozelluläres Karzinom imitieren [60, 61]. Die arterielle Phase hilft nicht nur bei der diagnostischen Unterscheidung zwischen einem HCC und einem iCCA, sondern auch bei der klareren Abgrenzung der vaskulären Anatomie vor der chirurgischen Resektion [56-58].

Im Vergleich zur MRT ist die kontrastverstärkte CT nur begrenzt in der Lage, die Ausbreitung des Tumors entlang der Gallengänge zu erkennen [62]. Die MRT zeichnet sich bei dieser Aufgabe durch ihren überlegenen Weichteilkontrast aus und gilt daher als das bildgebende Verfahren der Wahl für die Diagnose und das Staging des Cholangiokarzinoms. Ihre Genauigkeit ist vergleichbar mit der Genauigkeit der kontrastverstärkten CT und der direkten Cholangiografie in Kombination [62]. Ein optimales Protokoll für die Beurteilung von Cholangiokarzinomen sollte MRCP, konventionelle T1- und T2-gewichtete abdominelle MRT-Sequenzen (einschließlich T1 „In- und Out-of-phase“-Bildgebung), diffusionsgewichtete Bildgebung (DWI) und mehrphasige kontrastverstärkte Sequenzen in der arteriellen, portalen, venösen und verzögerten Phasen umfassen $[58,62]$. Die dynamischen Sequenzen können zu vorher festgelegten Zeitpunkten oder mittels Bolus-Tracking-Technik angefertigt werden [58]. Im MRT erscheinen iCCA in nativen T1-gewichteten 
Sequenzen hypointens und auf T2-gewichteten Sequenzen hyperintens [63-65]. In T2-gewichteten Bildern kann sich auch eine zentrale Hypointensität zeigen, die einem Fibrosegebiet entspricht. Dynamische kontrastverstärkte Sequenzen zeigen eine periphere Hyperintensität in der arteriellen Phase, gefolgt von einer progressiven und konzentrischen Auffüllung des Tumors mit Kontrastmittel. Das Kontrastmittelpooling in der Spätphase ist ein Hinweis auf eine Fibrose und deutet auf eine iCCA hin.

Die Magnetresonanztomografie mit Cholangio-Pankreatografie (MRT/MRCP) ist hilfreich, um das Gallengangsystem und die Gefäßstrukturen zu visualisieren und dadurch die anatomische Ausdehnung des Tumors genauer zu bestimmen. Die MRCP ist eine kontrastfreie MR-Technik. Dabei wird der T2-gewichtete Kontrast zwischen Galle (langes T2) und angrenzendem Gewebe (kurzes T2) durch die Verwendung stark T2-gewichteter Sequenzen akzentuiert. Die dünne Multi-Slice-MRCP ermöglicht eine hochauflösende Visualisierung über dreidimensionale Bilddatensätze [62]. Im Gegensatz zur endoskopischen retrograden Cholangiopankreatografie ist die MRCP nichtinvasiv und erlaubt die Visualisierung der Gallenwege proximal einer Obstruktion [58]. In Vorbereitung auf die MRCP sollen die Patienten mindestens 4 Stunden lang fasten, um Darmperistaltik und Magensekretion zu minimieren und die Gallenblasenauftreibung zu maximieren. Negative Kontrastmittel können hinzugefügt werden, um das Flüssigkeitssignal in Magen und Zwölffingerdarm zu reduzieren. Die DWI kann die MRCP bei der Erkennung von Tumoren in erweiterten oder verschlossenen Gallengängen unterstützen, wenn eine Kontrastmittelinjektion nicht möglich ist [62]. Es ist gut dokumentiert, dass die DWI die diagnostische Sensitivität der MRT für das Cholangiokarzinom erhöht. Frühere Studien haben eine Überlappung der dynamischen Kontrastverstärkungsmuster von kleinen raumfordernden intrahepatischen Cholangiokarzinomen $(<3 \mathrm{~cm})$ und Hepatozellulären Karzinomen dokumentiert [40, 50, 66, 67]. In solchen Fällen kann eine diffusionsgewichtete Bildgebung, die bei einer Zahl verschiedener b-Werte im Bereich von 0-800 s/ $\mathrm{mm}^{2}$ durchgeführt wird, helfen, das iCCA von HCC zu unterscheiden [68-71]. In ähnlicher Weise kann die DW-MRT helfen, benigne von malignen Strikturen zu unterscheiden, was für die Diagnose periduktal infiltrierender Subtypen des iCCAs von entscheidender Bedeutung ist [58, 72]. Im Allgemeinen tendieren die ADC-Werte von iCCAs dazu, signifikant niedriger zu sein als die des benachbarten normalen Leberparenchyms, wie es bei den meisten bösartigen Lebertumoren der Fall ist. Die Dokumentation einer Reihe von ADC-Werten, die spezifisch für iCCAs sind, wurde durch die große Variabilität der berichteten ADCWerte eingeschränkt. Diese Variabilität, die weitgehend auf technische Unterschiede der Bildakquirierung zurückzuführen ist, hat Forscher veranlasst, sich für die Verwendung normalisierter ADC-Werte zur optimalen quantitativen Charakterisierung von Leberläsionen, einschließlich des iCCA, einzusetzen. Studien haben dennoch gezeigt, dass die DW-MRI im Vergleich zu anderen MRT-Sequenzen trotz der hohen Varianz der b-Werte eine hohe diagnostische Genauigkeit für iCCAs aufweist. In einer Studie waren alle Cholangiokarzinome bei $b=0 \mathrm{~s} / \mathrm{mm}^{2}$ sichtbar, und die Mehrheit blieb bei der DWMRT bei steigenden b-Werten hyperintens, was darauf hindeutet, dass die Verwendung des früheren b-Werts in MR-Protokollen zur Erkennung von Cholangiokarzinomen in Betracht gezogen werden sollte. Dieselbe Studie legte nahe, dass die Normalisierung auf das LeberHintergrundparenchym zu einer minimalen Variabilität der ADCWerte im Vergleich zu anderen Indexorganen wie der Milz führte [69, 70]. Der Grad der Diffusionsbeschränkung im DW-MRI kann somit als unabhängiger präoperativer prognostischer Marker bei Patienten mit iCCA dienen. In einer anderen Studie zeigten Patienten, bei denen weniger als ein Drittel des Tumors eine Diffusionsrestriktion aufwies, im Vergleich zu Patienten, bei denen mehr als ein Drittel des Tumors eine Diffusionsrestriktion aufwies, ein fortgeschritteneres Baseline-TNM-Stadium, eine häufigere lymphatische Invasion und Lymphknotenmetastasen sowie eine häufigere stromale Metaplasie. Sowohl das krankheitsfreie als auch das Gesamtüberleben waren in der ersten Patientengruppe im Vergleich zur zweiten Gruppe signifikant niedriger $[58,73,74]$.

\begin{tabular}{|c|c|c|}
\hline 4.13) & Evidenzbasierte Empfehlung & Neu 2021 \\
\hline $\begin{array}{l}\text { Empfeh- } \\
\text { lungsgrad } \\
\text { A }\end{array}$ & \multicolumn{2}{|c|}{$\begin{array}{l}\text { Zur initialen Diagnostik und zum Staging bei kura- } \\
\text { tiver Intention eines Cholangiokarzinoms sollen } \\
\text { eine mehrphasische hepatische MRT-Untersuchung } \\
\text { sowie ein kontrastverstärktes CT des Thorax und } \\
\text { des Abdomens* vorliegen. } \\
{ }^{*} \text { Wenn komplettes MRT-Abdomen vorliegt, muss } \\
\text { kein CT des Abdomens ergänzt werden. }\end{array}$} \\
\hline \multirow[t]{2}{*}{$\begin{array}{l}\text { Level of } \\
\text { Evidence } \\
\mathbf{1}\end{array}$} & \multicolumn{2}{|c|}{ Primärrecherche: Zhang 2015[75] } \\
\hline & Konsens & \\
\hline
\end{tabular}

Die Rolle der 18F-FDG-PET bei der Diagnose und dem Staging von Patienten von ICCAs wurde bis dato kontrovers diskutiert. In einer neuen Metaanalyse wurde die Rolle der 18F-FDG-PET für Staging und Re-Staging bei insgesamt 2125 Patienten aus 47 Studien untersucht [76]. Sensitivität (Se) und Spezifität (Sp) der 18FFDG-PET in der Initialdiagnose betrugen je 91,7\% (95\%-KI 89,8; $93,2)$ bzw. 51,3\% (95\%-KI 46,4; 56,2), für einen Lymphknotenbefall Se $88,4 \%$ (95\%-KI 82,6; 92,8) und Sp 69,1\% (95\%-KI 63,8; $74,1)$, für das Vorliegen von Fernmetastasen Se 85,4\% (95\%-KI 79,5; 90,2) und Sp 89,7\% (95\%-KI 86,0; 92,7). Bei einem Verdacht auf ein Rezidiv betrug die Se 90,1\% (95\%-KI 84,4; 94,3) und die Sp 83,5\% (95\%-KI 74,4; 90,4). Somit weisen diese aktualisierten Daten darauf hin, dass der Einsatz von 18F-FDG-PET für das Staging (Lymphknoten und Fernmetastasen) und die Identifizierung von Rezidiven bei selektierten Patienten mit CCA für die Therapiestratifizierung sinnvoll sein kann, insbesondere wenn die Identifizierung okkulter Krankheitsherde das therapeutische Vorgehen verändern würde oder wenn die Diagnose eines Rezidivs nach der Standardbildgebung weiterhin unklar bleibt. Insofern kann der Einsatz der 18F-FDG-PET bei CCA nach interdisziplinärer Tumorboardempfehlung für Staging und Re-Staging indiziert sein. 
4.3.2. Untersuchungsmethoden zur Darstellung der maximalen Ausbreitung des Tumors

\begin{tabular}{|l|l|}
\hline 4.14) & Konsensbasiertes Statement \\
\hline EK & $\begin{array}{l}\text { Für die Erfassung der maximalen Ausbreitung des } \\
\text { Tumors inklusive Gefäßinvasion soll, wenn eine kurative } \\
\text { Behandlungsoption besteht, mindestens ein dynami- } \\
\text { sches kontrastverstärktes MRT eingesetzt werden. }\end{array}$ \\
\hline & starker Konsens \\
\hline
\end{tabular}

Insbesondere vor kurativen Resektionen oder minimalinvasiven interventionellen Therapien ist eine exakte Erfassung der maximalen Ausbreitung des Tumors sowie des Bezugs zu allen anatomisch wichtigen Strukturen unabdingbar.

Die MRT zeichnet sich bei dieser Aufgabe durch ihren überlegenen Weichteilkontrast aus und gilt daher als das bildgebende Verfahren der Wahl für die Diagnose der maximalen Ausdehnung des Cholangiokarzinoms [62]. Ein optimales Protokoll für die Beurteilung der maximalen Ausdehnung des Cholangiokarzinoms sollte MRCP, konventionelle T1- und T2-gewichtete abdominelle MRT-Sequenzen (einschließlich T1 „In- und Out-of-phase“-Bildgebung), diffusionsgewichtete Bildgebung (DWI) und mehrphasige kontrastverstärkte Sequenzen in der arteriellen, portalen, venösen und verzögerten Phase umfassen [58, 62]. Für die weiteren Details siehe bitte auch das Kapitel „Bildgebende und/oder endoskopische Untersuchungen zum Staging und zur Diagnosestellung eines biliären Karzinoms „ S. 203. Neben dem Einsatz der MRCP und diffusionsgewichteter MRT-Sequenzen kann die Nachkontrastdarstellung mit traditionellen, extrazellulären Kontrastmitteln auf Gadoliniumbasis (Gd-DTPA) oder Derivaten wie Gadoliniummethoxybenzyldiethylentriamin-Penta-Essigsäure (Gd-EOB-DTPA) genauere Informationen bezüglich der Tumorausdehnung liefern.

Gd-EOB-DTPA kombiniert die Eigenschaften eines herkömmlichen extrazellulären Kontrastmittels auf Gadoliniumbasis mit denen von hepatozytenspezifischen Kontrastmitteln. Frühere Studien haben die Überlegenheit von Gd-EOB-DTPA beim Nachweis und bei der Charakterisierung von Leberläsionen bei Patienten mit diffusen Lebererkrankungen dokumentiert. Da die Nachkontrastsignalintensität der Leber bei Verwendung hepatozytenspezifischer Kontrastmittel wie Gd-EOB-DTPA im Vergleich zu herkömmlichen extrazellulären Kontrastmitteln auf Gadoliniumbasis höher ist, werden Cholangiokarzinome sowohl bei frühen als auch bei verzögerten Phasensequenzen als hypointens sichtbar [77]. Dadurch entsteht ein scharfer Kontrast zwischen der Läsion und dem Leberhintergrund, was eine genauere Beurteilung der Tumorausdehnung sowie des Vorhandenseins assoziierter Satellitenläsionen ermöglicht, die in 10-20\% der Fälle von metastasiertem CCA gesehen werden [77]. Die erhöhte Sichtbarkeit von Cholangiokarzinomen in dieser Umgebung ist besonders hilfreich für Patienten mit einem Hintergrund diffuser Lebererkrankungen, bei denen metastasierte CCA nach Verabreichung traditioneller extrazellulärer Kontrastmittel auf Gadoliniumbasis atypische Enhancementmuster aufweisen können [58, 78]. Bei der Gd-EOB-DTPA können auch die relative Signalintensität der Leber und die Sichtbarkeit der Gallenwege auf der hepatobiliären Phase als quantifi- zierbare Surrogatmarker der Gallenfunktion dienen. Gd-EOBDTPA wird von Hepatozyten aufgenommen und in das Gallensystem ausgeschieden. Eine verminderte Signalintensität der Hintergrundleber und eine verringerte Sichtbarkeit der Gallenwege in der hepatobiliären Phase weisen auf eine gestörte Gallenfunktion hin und korrelieren quantitativ mit dem Gesamtbilirubinspiegel und könnten somit ein ergänzender Leberfunktionsparameter vor ausgedehnten Resektionen sein [77].

\subsubsection{Diagnostikalgorithmus}

\begin{tabular}{|c|c|c|}
\hline 4.15) & Evidenzbasierte Empfehlung & Neu 2021 \\
\hline $\begin{array}{l}\text { Empfeh- } \\
\text { lungsgrad } \\
\text { A }\end{array}$ & \multicolumn{2}{|c|}{$\begin{array}{l}\text { Bei Verdachtsdiagnose auf ein Cholangiokarzinom } \\
\text { soll eine Schnittbildgebung zur Beurteilung der } \\
\text { Tumorausdehnung verwendet werden. }\end{array}$} \\
\hline \multirow[t]{2}{*}{$\begin{array}{l}\text { Level of } \\
\text { Evidence } \\
\mathbf{1}\end{array}$} & \multicolumn{2}{|c|}{ Primärrecherche: Zhang 2015 [75] } \\
\hline & starker Konsens & \\
\hline
\end{tabular}

Die Diagnose eines Cholangiokarzinoms basiert auf kontrastverstärkten bildgebenden Untersuchungen und histologischen Analysen. Der Diagnosealgorithmus eines Patienten mit Verdacht auf ein Cholangiokarzinom ist in der Abb. 1 dargestellt. Aufgrund der erhöhten Ansprüche bezüglich der lokalen Tumorausdehnung wird nach dem Staging des Thorax sowie Abdomens mittels CT ein je nach Subtyp spezifiziertes MRT präoperativ empfohlen.

\subsubsection{Endoskopische Diagnostik}

\begin{tabular}{|c|c|c|}
\hline 4.16) & Evidenzbasierte Empfehlung & Neu 2021 \\
\hline $\begin{array}{l}\text { Empfeh- } \\
\text { lungsgrad } \\
\mathbf{0}\end{array}$ & \multicolumn{2}{|c|}{$\begin{array}{l}\text { Der endoskopische Ultraschall kann zu Diagnose, } \\
\text { lokalem Staging und Gewebegewinnung beim } \\
\text { biliären Karzinom verwendet werden. }\end{array}$} \\
\hline $\begin{array}{l}\text { Level of } \\
\text { Evidence } \\
\mathbf{1}\end{array}$ & \multicolumn{2}{|c|}{ Primärrecherche: De Moura 2018 [79] } \\
\hline & \multicolumn{2}{|l|}{ starker Konsens } \\
\hline
\end{tabular}

Die endosonografiegestützte Feinnadelaspirationszytologie (EUS-FNA) hatte in einer Metaanalyse von 6 Studien, die z. T. auch PSC-Patienten einschlossen, eine Sensitivität von $66 \%$ und eine Spezifität von 100 \% für die Diagnose eines CCA [70]. Auch bei Fehlen einer Läsion in der Schnittbildgebung konnte noch eine Sensitivität von 45 \% erreicht werden. Bei Patienten mit negativer Bürstenzytologie konnte aus 3 Studien eine Sensitivität von $59 \%$ (Spezifität 100\%) errechnet werden. Damit ist die EUS-FNA eine valide Methode auch und insbesondere dann, wenn eine histologische Bestätigung erforderlich ist. Einschränkend sei eine Studie erwähnt, bei der von 191 Patienten i. R. eines neoadjuvanten Therapieprotokolls vor Lebertransplantation bei 16 eine perkutane $(n=13)$ oder EUS-gesteuerte $(n=3)$ Gewebegewinnung erfolgte [80]. Bei 5 von 6 Patienten mit definitiv maligner Histologie traten peritoneale (Stichkanal-)Metastasen auf (die Verteilung 
perkutan vs. EUS-FNA ist nicht aufgeführt, vs. 14/175 ohne Biopsie). Dies konnte in einer jüngeren Studie an 150 Patienten, von denen 61präoperativ EUS-gesteuert biopsiert worden waren, nicht nachvollzogen werden[81].

\begin{tabular}{|l|l|}
\hline $\mathbf{4 . 1 7})$ & Konsensbasierte Empfehlung \\
\hline EK & $\begin{array}{l}\text { Wenn im Rahmen einer ERCP der V. a. ein extrahepati- } \\
\text { sches Cholangiokarzinom gestellt wird, sollte im Rah- } \\
\text { men einer ERCP eine Zangenbiospie oder eine Bürsten- } \\
\text { zytologie entnommen werden. }\end{array}$ \\
\hline & starker Konsens \\
\hline
\end{tabular}

Die endoskopische Bürstenzytologie während der ERC hat in verschiedenen Studien eine Sensitivität von 30-78\% [82-84] (Anm: Review, höchste und niedrigste Sensitivität, bei gleichen Werten jeweils neueste) und eine Spezifität von 90-100\% [85, 86]. Der positive Prädiktivwert lag bei 94-100\%, der negative Prädiktivwert jedoch nur bei 8-62\% [86, 87]. In einer Metaanalyse zu Studien, die die Bürstenzytologie und die transpapilläre Biopsie verglichen, betrug die kombinierte Sensitivität und Spezifität der Bürstenzytologie zur Diagnose einer malignen Gallengangsstenose $45 \%$ (95\%-KI 40-50\%) und 99\% (98-100\%) [40]. Bei Patienten mit PSC war in einer Metaanalyse zu 11 retrospektiven und prospektiven Studien mit insgesamt 747 Patienten die Sensitivität der Bürstenzytologie für ein CCA $43 \%$ (35-52\%), die Spezifität 97\% (95-98\%) [88]. Damit sichert die Bürstenzytologie im Falle eines Nachweises die Diagnose eines biliären Karzinoms, ist jedoch bei negativer Histologie nicht zum Ausschluss eines biliären Karzinoms geeignet.

Die endobiliäre, transpapilläre Zangenbiopsie während der ERC hat in verschiedenen Studien eine Sensitivität von 29-81\% [82, 89, 90] und eine Spezifität von 90-100\% [91, 92]. Der positive Prädiktivwert lag bei 94-100\% [91, 92], der negative Prädiktivwert jedoch nur bei 31-81\% [91, 92].

In der bereits oben aufgeführten Metaanalyse [40] war die kombinierte Sensitivität und Spezifität der transpapillären Biopsie zur Diagnose einer malignen Gallengangstenose 48,1\% (95\%-KI $42,8-53,4 \%$ ) und $99,2 \%(97,6-99,8 \%)$, lag somit geringgradig höher als die der Bürstenzytologie. Die diagnostische Genauigkeit ist für CCAs etwas höher als für das Pankreaskarzinom, am ehesten aufgrund des oberflächlicheren, somit besser zugänglichen Tumorwachstums des CCA. Ähnlich wie für die Bürstenzytologie gilt für die Zangenbiopsie, dass bei positiver Histologie die Diagnose eines biliären Karzinoms zwar gesichert ist, bei negativer Histologie jedoch nicht ausgeschlossen werden kann. Gallengangperforationen durch die Zangenbiopsien wurden beschrieben $[93,94]$, jedoch insgesamt nur selten berichtet.

Die Kombination von Bürstenzytologie und transpapillärer endobiliärer Zangenbiopsie wurde in 6 Studien untersucht [40]. Sie kann die Sensitivität der Diagnose einer malignen Striktur mit einer kombinierten Sensitivität von 59,4\% (53,7-64,8\%) erreichen, die Spezifität bleibt hoch (100\% (98,8-100,0\%)). Somit wird die diagnostische Genauigkeit durch die Kombination beider Verfahren zur Histologiegewinnung gesteigert, jedoch nicht in dem Maße, dass ein Ausschluss eines Karzinoms durch die Histologie in sicherer Weise möglich ist.

\begin{tabular}{|l|l|l|}
\hline $\mathbf{4 . 1 8}$ & Evidenzbasiertes Statement & Neu 2021 \\
\hline $\begin{array}{l}\text { Level of } \\
\text { Evidence }\end{array}$ & $\begin{array}{l}\text { Bei V. a. ein extrahepatisches Cholangiokarzinom } \\
\text { kann durch Einsatz der Cholangioskopie in Kombi- } \\
\text { nation mit visuell gezielter Biopsie die Sensitivität } \\
\text { der Diagnose gesteigert werden. }\end{array}$ \\
\hline & Primärrecherche: Navaneethan 2015 [95] \\
\hline & starker Konsens \\
\hline
\end{tabular}

Die meisten Studien zum Einsatz der Cholangioskopie verwendeten die Single-Operator-Cholangioskopie (mit SpyGlass ${ }^{\circledR}$ ). In einer systematischen Übersicht [95] wurden insgesamt 10 Studien identifiziert, die die Cholangioskopie mit visuell-endoskopisch gezielter Biopsieentnahme bei Gallengangsstrikturen evaluierten. Die kombinierte Sensitivität der so gewonnenen Histologie zur Diagnose maligner Strikturen war 60,1\% (95\%-KI 54,9-65,2\%) bei einer Spezifität von 98,0\% (96,0-99,0\%). Etwas bessere Werte ergaben sich, wenn nur die Biopsien bei CCA ausgewertet wurden (Sensitivität 66,2\% (59,7-72,3\%), Spezifität 97,0\% (94,0$99,0 \%)$ ).

Die Hinzunahme des visuell-endoskopischen Eindrucks konnte die Sensitivität erheblich steigern (84,5\% (79,2-88,9\%)), allerdings zulasten der Spezifität $(82,6 \%(77,1-87,3 \%))$. Letztlich sind die endoskopischen Kriterien für Malignität nicht abschließend evaluiert, insbesondere vor dem Hintergrund entzündlicher Veränderungen (z. B. bei PSC). Interessant ist der Einsatz der Cholangioskopie bei Strikturen mit zuvor nicht eindeutiger Histologie durch Bürste u./o. Biopsie: Damit konnte dennoch mit einer Sensitivität von $67,3 \%(52,5-80,1 \%)$ und einer Spezifität von 93,3\% $(83,1-98,7 \%)$ ein CCA diagnostiziert werden. Bei direktem Vergleich zwischen Bürstenzytologie, Zangenbiopsie und Cholangioskopie mit Biopsieentnahme war die Cholangioskopie den beiden anderen Verfahren in Sensitivität, Genauigkeit und negativem Prädiktivwert signifikant überlegen [89].

Der Einsatz der nächsten Gerätegeneration oder die Verwendung der direkten peroralen Cholangioskopie könnte theoretisch sowohl die endoskopische Visualisierung als auch Größe und Zahl der Biopsien verbessern. Dazu liegen noch keine vergleichenden Studien mit älteren Cholangioskopen vor. Eine 2019 publizierte Studie hat randomisiert die konventionelle Bürstenzytologie-Entnahme mit der digitalen Cholangioskopie mit visuell gestützter Biopsie verglichen. Dabei war die Sensitivität der cholangioskopiegesteuerten Biopsie signifikant höher als die der Bürstenzytologie (68,2\% vs. $21,4 \%)$, ebenfalls die Sensitivität der visuellen Einschätzung $(95,5 \%$ vs. $66,7 \%)$ und die Genauigkeit insgesamt $(87,1 \%$ vs. $65,5 \%$ ), bei allerdings nicht gesteigertem PPV (positiv predictive value) und NPV (negativ predictive value) [96]. Zusätzliche Methoden zur Verbesserung der Visualisierung biliärer Veränderungen wie die Chromoendoskopie, virtuelle Chromoendoskopie oder die Verwendung sondenbasierter Endomikroskopiesystem sind in Einzelstudien gut evaluiert, jedoch nicht in der Routinediagnostik verfügbar. Grundsätzlich sind all die genannten Verfahren der 
Bürstenzytologie, Biopsie und Cholangioskopie auch perkutan, z. B. über einen PTCD-Zugang, einsetzbar.

\begin{tabular}{|l|l|l|}
\hline $\mathbf{4 . 1 9 )}$ & Konsensbasierte Empfehlung & Neu 2021 \\
\hline EK & $\begin{array}{l}\text { Patienten mit primär sklerosierender Cholangitis und } \\
\text { Erstmanifestation einer dominanten Stenose sollen } \\
\text { mittels MRT/MRCP und ERCP/Histologie weiter abgeklärt } \\
\text { werden. } \\
\text { Bei weiterbestehendem Verdacht auf ein CCA trotz } \\
\text { negativer Diagnostik sollte eine kurzfristige erneute } \\
\text { Reevaluation, ggf. mit Wiederholung der Untersuchun- } \\
\text { gen, oder bei therapeutischer Relevanz eine Klärung im } \\
\text { Rahmen einer explorativen Laparotomie erfolgen. }\end{array}$ \\
\hline & Konsens \\
\hline
\end{tabular}

Zur Überwachung von Patienten mit PSC darf auf die „S2k-LL Autoimmune Lebererkrankungen “ [35] der DGVS verwiesen werden, die schreibt: „Die Unterscheidung der benignen von der malignen Stenose ist eines der klinisch relevantesten Probleme bei Patienten mit PSC. Es sollten möglichst verschiedene Verfahren angewendet werden, um den Verdacht eines CCA weitestgehend zu bestätigen oder auszuschließen. Eine Cholangioskopie wird von einigen Zentren insbesondere zur gezielten Gewebeentnahme favorisiert. Auch eine Wiederholung bereits erfolgter Untersuchungen erzielt in manchen Fällen eine Diagnosesicherung. “ Eine ausführliche Aufstellung zur Genauigkeit der Bürstenzytologie zur Diagnose des CCA bei Patienten mit PSC in Einzelstudien und in Reviews findet sich außerdem in der ESGE-Leitlinie „Role of Endoscopy in Primary Sclerosing Cholangitis“ [97] ( $\triangleright$ Tab. 7, 8), zusammenfassend s. u. Kapitel Bürstenzytologie. Interessant ist, dass in einer kürzlich publizierten deutschen Studie unter Patienten mit PSC diejenigen Patienten, die eine regelmäßige Dilatation dominanter Stenosen erhielten, mit 5,3\% $(n=7)$ zwar nicht signifikant $(p=0,1)$, aber möglicherweise doch relevant seltener ein CCA entwickelten als die Patienten, die nur bei Beschwerden dilatiert wurden, mit 9,8\% $(n=15)$ [74]. Ob dies ein Effekt der verminderten Zahl von Cholangitis-Episoden ist (i. S. einer Unterbrechung der Inflammations-KarzinomSequenz), muss abgewartet werden.

Bei hochgradigem Verdacht auf ein biliäres Karzinom (iCCA, pCCA, dCCA) und als resektabel eingeschätztem Befund muss eine histologische Sicherung nicht regelhaft präoperativ erfolgen, da ein fehlender Tumornachweis in der Histologie/Zytologie aufgrund der niedrigen Sensitivität das therapeutische Vorgehen nicht verändert. Häufig wird die Erstdiagnose eines pCCA oder dCCA bei der ERC zur Ableitung bei Cholestase gestellt, dann kann ggf. eine Bürstenzytologie u./o. Zangenbiopsie zur Histologiegewinnung eingesetzt werden. Sensitivität und Spezifität der Verfahren zur histologischen Sicherung in Studien war in hohem Maße abhängig von der Prätestwahrscheinlichkeit der Studienpopulation (z. B. PSC- vs. Non-PSC-Patienten, Patienten mit unklarer Striktur vs. Patienten mit jeglicher Striktur, symptomatische vs. asymptomatische Striktur, nur dCCA vs. dCCA und Papillen- und Pankreaskarzinom). Bei unklaren Befunden kann die Cholangio- skopie mit endoskopisch-visuell gezielter Biopsieentnahme eingesetzt werden, die die diagnostische Ausbeute erhöht (s. u.).

Bei Verdacht auf IgG4-assoziierte Cholangitis kann die bioptische Sicherung die probatorische Einleitung einer spezifischen Therapie begründen. Bei Patienten mit Primär Sklerosierender Cholangitis (PSC) empfiehlt die DGVS-Leitlinie „Autoimmune Lebererkrankungen“ bei dominanter Stenose eine histologische Sicherung, bei weiterhin bestehendem Verdacht auf ein CCA trotz negativer Histologie eine kurzfristige Reevaluation [35]. Vor Einleitung einer palliativen Chemotherapie ist die histologische Sicherung der Tumorentität erforderlich, die im Rahmen einer ERC oder durch perkutane oder endosonografische Punktion erfolgen kann (s.u.). Ob in Zukunft der Einsatz neoadjuvanter (Chemo-)Therapieansätze häufiger eine histologische Sicherung auch bei resektablem Befund erforderlich machen wird, bleibt aktuell ebenso spekulativ wie das Potenzial repetitiver Biopsien zur Identifikation eines molekular gestützten Therapieansatzes in der palliativen Situation.

Hinsichtlich der histopathologischen Sicherung s. auch das Kapitel „Histopathologische und molekulare Diagnostik“, S. 199.

\subsection{Operative und interventionelle Therapieverfahren}

\subsubsection{Resektion}

\begin{tabular}{|c|c|c|}
\hline 4.20) & Konsensbasierte Empfehlung & Neu 2021 \\
\hline EK & \multicolumn{2}{|c|}{$\begin{array}{l}\text { Eine Resektion eines pCCA, dCCA oder iCCA soll } \\
\text { erfolgen, wenn eine komplette Resektion (R0-Resektion) } \\
\text { möglich erscheint. }\end{array}$} \\
\hline & starker Konsens & \\
\hline
\end{tabular}

Die radikale chirurgische Entfernung allen Tumorgewebes stellt gegenwärtig die einzige kurative Behandlung des nicht fernmetastasierten iCCA und pCCA dar. Multifokalität (bei iCCA), Lymphknotenmetastasen (N1) und eine Gefäßinvasion sind die wichtigsten prognoserelevante Faktoren, stellen jedoch keine Kontraindikation dar, sofern eine R0-Resektion erreichbar scheint [98-104]. Häufig erfordert die chirurgische Behandlung von iCCA und pCCA ausgedehnte Leberresektionen [105-107]. Postoperativ sollte eine adjuvante Therapie erfolgen (siehe BILCAP-Studie (Capecitabine compared with observation in resected biliary tract cancer)) [108]. Bei initial irresektablem oder sogenanntem borderline-resektablem iCCA kann nach einer Downsizing-Therapie eine Resektion erwogen werden [109].

\begin{tabular}{|l|l|l|}
\hline $\mathbf{4 . 2 1 )}$ & Konsensbasierte Empfehlung & Neu 2021 \\
\hline EK & $\begin{array}{l}\text { Eine Resektion eines Gallenblasenkarzinoms soll erfol- } \\
\text { gen, wenn klinisch keine Fernmetastasen vorliegen } \\
(\mathrm{cM} 0) \text { und eine komplette Resektion (R0-Resektion) } \\
\text { möglich erscheint. }\end{array}$ \\
\hline & starker Konsens \\
\hline
\end{tabular}


Die langfristige Prognose des Galleblasenkarzinoms ist insgesamt sehr schlecht, mit einer 5-Jahre-Überlebensrate von 5-15\%. Wenn der Krebs jedoch in einem frühen Stadium erkannt und angemessen behandelt wird, können 5-Jahres-Überlebensraten von $75 \%$ erreicht werden $[110,111]$. Es besteht ein internationaler Konsens darüber, dass die R0-Resektion der stärkste prognostische Faktor für das Langzeitergebnis und die Heilungschancen bei Patienten mit Gallenblasenkarzinom ist [111]. In diesem Zusammenhang bestimmt die Tiefe der Invasion durch die Gallenblasenwand die chirurgische Standardbehandlung des Gallenblasenkarzinoms [110-112].

Bei Tis- und T1a-Tumoren ist eine Cholezystektomie ohne weitere Resektion erforderlich [111]. Beim Gallenblasenkarzinom der Kategorie $\geq \mathrm{T} 1 \mathrm{~b}$ ist eine zusätzliche Leberresektion mit systematischer Lymphadenektomie indiziert, sofern der Patient für die Operation geeignet ist. Sowohl die Gallenblasenbettresektion als auch die Segmentresektion IVb und V ist ein onkologisch akzeptables Verfahren, vorausgesetzt, es wird eine R0-Resektion durchgeführt. Eine erweiterte Hepatektomie ist in der Regel bei Patienten mit lokal fortgeschrittenem Tumor mit biliärer und vaskulärer Beteiligung erforderlich, um eine R0-Resektion zu erreichen [110, 111].

Eine routinemäßige Resektion der Hauptgallengänge ist weder indiziert noch empfohlen, da sie die postoperative Morbidität erhöht, die Zahl der entfernten Lymphknoten nicht erhöht und nicht mit einer Verbesserung des Gesamtüberlebens assoziiert ist. Eine Gallengangresektion ist nur in Fällen indiziert, in denen ein positiver zystischer Ductusrand zum Zeitpunkt der ursprünglichen Resektion beseitigt werden muss, bei Gallenblasenkrebs mit direkter Infiltration des hepatoduodenalen Ligaments und in Fällen mit intensiver postoperativer Fibrose mit signifikanter Lymphadenopathie des hepatoduodenalen Ligaments, um eine adäquate Lymphadenektomie zu ermöglichen [111].

Beim Gallenblasenkarzinom der Kategorie $\geq 1$ b erfordert die „Standard“-Lymphknotendissektion die Entnahme von mindestens 6 Lymphknoten und umfasst N1 (zystische 12c, pericholedochale $12 \mathrm{~b}$, Hilusknoten $12 \mathrm{~h}$, Knoten der eigentlichen Leberarterie 12a) und N2 (peripankreatische 13a, periportale $12 \mathrm{p}$, periduktale und gemeinsame Leberarterie). Eine Skelettierung der Leberarterie, der Pfortader und des Gallengangs wird empfohlen. Das Befallen von Lymphknoten aus Truncus coeliacus und para-aortal sollte als M1-Krankheit betrachtet werden, und die Entnahme dieser Lymphknoten ist nicht mit einem verbesserten Überleben assoziiert [111].

\begin{tabular}{|l|l|l|}
\hline $\mathbf{4 . 2 2}$ & Konsensbasierte Empfehlung & Neu 2021 \\
\hline EK & $\begin{array}{l}\text { Wird bei einer Cholezystektomie intra- oder postoperativ } \\
\text { ein Carcinoma in situ (Tis) oder ein Mukosakarzinom } \\
\text { (T1a) festgestellt, sollte bei Vorliegen einer R0-Situation } \\
\text { (D. cysticus) keine Nachresektion erfolgen. }\end{array}$ \\
\hline & starker Konsens \\
\hline
\end{tabular}

Das inzidentelle Gallenblasenkarzinom ist definiert als ein Karzinom, das bei der histologischen Untersuchung der Probe nach Standard-Cholezystektomie entdeckt wird, da frühe Gallenblasenkarzinome keine spezifischen Symptome aufweisen. Das inzidentelle Gallenblasenkarzinom repräsentiert etwa $70 \%$ der Gallenblasenkarzinome in nicht endemischen Gebieten und tritt bei 0,2-3\% der Patienten auf, die sich einer Cholezystektomie unterziehen.

Ein Gallenblasenkarzinom der Kategorie T1a ist definiert als Karzinom, das auf die Schleimhaut beschränkt ist, und T1b als Karzinom, das auf die Muscularis-Schleimhaut beschränkt ist. Patienten mit einem auf die Schleimhaut beschränkten Karzinom (T1a oder weniger) zeigten 5-Jahres-Überlebensraten von bis zu $100 \%$ nach alleiniger Cholezystektomie. Deshalb wird bei Patienten mit inzidentellem Gallenblasenkarzinom der Kategorie Tis und T1a eine einfache Cholezystektomie empfohlen. Eine erweiterte Resektion ist nicht erforderlich. [110, 111, 113]

\begin{tabular}{|l|l|l|}
\hline $\mathbf{4 . 2 3}$ & Konsensbasierte Empfehlung & Neu 2021 \\
\hline EK & $\begin{array}{l}\text { Bei intra- oder postoperativem Nachweis eines Gallen- } \\
\text { blasenkarzinoms der Kategorie } \geq \text { T1b soll bei kurativem } \\
\text { Ansatz eine onkologische Resektion oder Nachresektion } \\
\text { erfolgen. }\end{array}$ \\
\hline & starker Konsens \\
\hline
\end{tabular}

Aktuelle Leitlinien für die Behandlung von inzidentellen Gallenblasenkarzinomen empfehlen eine Nachresektion bei T1b-, T2- und T3-Läsionen, es sei denn, dies ist aufgrund einer fortgeschrittenen Erkrankung oder eines schlechten Allgemeinzustands des Patienten kontraindiziert $[111,114]$.

Es besteht Konsens, dass die R0-Resektion der stärkste prognostische Faktor für das Langzeitergebnis und die Heilungschancen bei Patienten mit Gallenblasenkarzinom ist [111, 115]. Diesbezüglich zeigten Lee et al. in einer multivariaten Analyse bei Patienten mit einem T1b-Tumor, dass die R1/R2-Resektion und die Lymphknotenmetastasierung eine schlechte Prognose signifikant vorhersagten, wobei die 1-Jahres-Überlebensrate bei T1b-Tumoren, die sich keiner radikalen Exzision unterziehen, auf $50 \%$ sank [116].

Die Reoperation sollte so früh wie möglich durchgeführt werden, sobald das endgültige histopathologische Staging vorliegt, die Metastasenaufarbeitung abgeschlossen ist und der Patient für die Reoperation geeignet ist, die je nach Überweisungszeitpunkt und Krankheitsstadium 2-4 Wochen nach der Cholezystektomie erfolgen kann. Eine radikale Reoperation wird für Patienten mit der Krankheit $\geq$ pT1b empfohlen [111].

Eine radikale Cholezystektomie mit Lymphadenektomie sollte bei Patienten mit T1b-GBCA empfohlen werden, bei denen kein erhöhtes Risiko für postoperative Komplikationen besteht [113, 115].

Die Resektion der extrahepatischen Gallenwege ist die Standardoperation bei Gallenblasenkarzinomen, die (makroskopisch oder mikroskopisch) den Hals der Gallenblase und/oder den D. cysticus betreffen [111]. 


\begin{tabular}{|l|l|l|}
\hline 4.24) & Konsensbasierte Empfehlung & Neu 2021 \\
\hline EK & $\begin{array}{l}\text { Bei einem isolierten intrahepatischen Rezidiv eines CCA } \\
\text { kann eine erneute Resektion durchgeführt werden, } \\
\text { wenn eine komplette Resektion (R0-Resektion) möglich } \\
\text { erscheint. }\end{array}$ \\
\hline \begin{tabular}{l} 
Konsens \\
\hline
\end{tabular} \\
\hline
\end{tabular}

Nach Resektion eines iCCA kann bei einem auf die Leber beschränkten Tumorrezidiv ein erneuter Resektionsversuch unternommen werden. Die Überlebensraten sind denen nach Primäroperation vergleichbar $[117,118]$. Isolierte Rezidive eines perihilären Cholangiokarzinoms sind nur selten einer chirurgischen Therapie zugänglich.

\begin{tabular}{|l|l|l|}
\hline 4.25) & Konsensbasierte Empfehlung & Neu 2021 \\
\hline EK & $\begin{array}{l}\text { Wenn Rezidive nach einer vorangegangenen Operation } \\
\text { nicht erneut operativ versorgt werden können, können } \\
\text { diese mit thermischer Ablation behandelt werden, wenn } \\
\text { dadurch eine komplette Ablation möglich erscheint. }\end{array}$ \\
\hline & starker Konsens \\
\hline
\end{tabular}

Für die thermische Ablation bei iCCA Rezidiven nach Resektion konnten in mehreren Studien gute Ergebnisse gezeigt werden [119-127]. So konnte in 2 kontrollierten Studien bei insgesamt 230 Patienten [120, 121] bei vergleichbaren Patientencharakteristiken ein vergleichbares progressionsfreies Gesamtüberleben von 31,3 Monaten für die Resektion versus 29,4 Monaten für die Ablation [120] bei deutlich höherer Komplikationsrate für die Resektion im Vergleich zur Ablation (13,8\%, vs. 5,3\% in [120] und $46,9 \%$ vs. $3,9 \%$ in [121] erreicht werden. In einer weiteren Studie von Kim JH et al. [128] wurden insgesamt 20 Patienten mit 29 rezidivierenden iCCAs einer perkutanen RFA unterzogen. Alle Patienten hatten sich einer kurativen Resektion des primären iCCA unterzogen. Die Tumorgröße lag zwischen $0,7 \mathrm{~cm}$ und $4,4 \mathrm{~cm}$ in der maximalen Größe (Mittelwert 1,9 cm; Median $1,5 \mathrm{~cm}$ ). Die technische Effektivität von der Ablation betrug $97 \%$ (28/29) der rezidivierenden iCCAs. Das mittlere progressionsfreie Überleben des lokalen Tumors betrug 39,8 Monate, und die kumulative progressionsfreie 6-Monate- und 1-, 2- und 4-JahresÜberlebensrate betrug $93 \%, 74 \%, 74 \%$ und $74 \%$. Das mediane Gesamtüberleben nach Ablation betrug 27,4 Monate und die kumulative Gesamtüberlebensrate von 6 Monaten und 1, 2 und 4 Jahren betrug $95 \%, 70 \%, 60 \%$ und $21 \%$. Es gab in dieser Fallserie 2 Komplikationen (einen Leberabszess und eine biliäre Striktur, somit $7 \%$ pro Behandlung) während des Follow-ups, aber keine Todesfälle.

\subsubsection{Lebertransplantation}

\begin{tabular}{|l|l|l|}
\hline $\mathbf{4 . 2 6})$ & Konsensbasierte Empfehlung & Ne21 \\
\hline EK & $\begin{array}{l}\text { Eine Lebertransplantation für das iCCA soll außerhalb } \\
\text { von Studien nicht erfolgen. }\end{array}$ \\
\hline & starker Konsens \\
\hline
\end{tabular}

Derzeit stellt das iCCA eine Kontraindikation für eine Lebertransplantation in den meisten Ländern weltweit aufgrund früher Tumorrezidive und schlechter Überlebensraten (5-Jahres-Gesamtüberleben zwischen $35 \%$ und $47 \%$ ) dar [12, 61, 129].

Zu beachten ist, dass dies nicht immer Intention-to-Treat-Analysen sind, sondern die Diagnose eines iCCA vielfach erst nach der Lebertransplantation im finalen histologischen Befund der Explantatleber gestellt wird, die Lebertransplantation jedoch ursprünglich mit der Indikation eines HCCs durchgeführt wurde [130, 131].

Im Fall eines iCCA $<2$ cm (d. h. „sehr frühes“ iCCA) scheint die Lebertransplantation jedoch mit ähnlichen Ergebnissen wie bei einem HCC innerhalb der Mailand-Kriterien einherzugehen [132]. Dieses Konzept wurde durch eine weitere Studie validiert [133].

Lunsford et al. haben vor Kurzem ein Protokoll für die Lebertransplantation bei Patienten mit lokal fortgeschrittenem, inoperablem iCCA nach neoadjuvanter Chemotherapie erstellt. Voraussetzung war eine dauerhafte Regression oder zumindest kein Tumorprogress unter Chemotherapie. Sechs von 21 rekrutierten Patienten wurden transplantiert und hatten eine Gesamtüberlebensrate nach 5 Jahren von 83 \% (5 der 6 Patienten), 3 davon ohne Rezidiv [134].

\begin{tabular}{|c|c|c|}
\hline 4.27) & Evidenzbasierte Empfehlung & Neu 2021 \\
\hline $\begin{array}{l}\text { Empfeh- } \\
\text { lungsgrad } \\
\mathbf{0}\end{array}$ & \multicolumn{2}{|c|}{$\begin{array}{l}\text { Bei irresektablem, nichtmetastasiertem pCCA, das } \\
\text { die Mayo-Kriterien erfüllt, kann eine Lebertrans- } \\
\text { plantation unter Studienbedingungen erwogen } \\
\text { werden. }\end{array}$} \\
\hline \multirow[t]{2}{*}{$\begin{array}{l}\text { Level of } \\
\text { Evidence } \\
\mathbf{3}\end{array}$} & \multicolumn{2}{|c|}{$\begin{array}{l}\text { Primärrecherche: Becker } 2008 \text { [135], Darwish } 2012 \\
\text { [136] }\end{array}$} \\
\hline & starker Konsens & \\
\hline
\end{tabular}

\section{Mayo-Kriterien}

Irresektables pCCA oder pCCA in PSC-Zirrhose

Tumordurchmesser $<3 \mathrm{~cm}$

keine LK-Metastasen (obligate chirurgische Exploration)

keine extrahepatische Tumormanifestation

histologisch/zytologisch bestätigtes pCCA oder CA19-9>1000 kU/L mit Vorliegen radiologischer Zeichen einer malignen Stenose 
Die Lebertransplantation scheint bei irresektablen, nichtmetastasierten pCCA eine valide Option mit vielversprechenden Ergebnissen (Gesamtüberlebensrate $>50 \%$ nach 5 Jahren). Die Rolle der neoadjuvanten Therapie ist bislang nicht geklärt.

Die meisten Daten zur Lebertransplantation stammen aus den USA, u. a. aus dem Zentrum mit der größten Erfahrung, der Mayo Klinik in Rochester [137]. In diesem Zentrum wurde ein neoadjuvantes Protokoll etabliert, das auf einer Kombination aus Strahlentherapie (45 Gy external beam radiation mit ggf. intraluminaler Brachytherapie) und Chemotherapie (5-FU über 3 Wochen, gefolgt von Capecitabin) basiert. Lymphknotenmetastasen stellen eine absolute Kontraindikation dar. Sie sollen im Rahmen einer explorativen Laparotomie vor Lebertransplantation ausgeschlossen werden. Bis dato wurden mehr als 160 Patienten gemäß diesem Protokoll transplantiert mit einem 5-Jahres-Gesamtüberleben zwischen $50 \%$ und $80 \%$, abhängig von verschiedenen Unterund Risikogruppen [135, 136, 138, 139]. Eine prospektive, randomisierte Studie zum Vergleich Lebertransplantation versus palliative Therapie existiert bislang nicht.

In einer Analyse von Mantel et al. von ELTR-Daten (105 Patienten mit pCCA) konnte kein Nutzen durch eine neoadjuvante Therapie gefunden werden [140]. In einer Subgruppe von allerdings nur 28 Patienten, die die Mayo-Auswahlkriterien erfüllten (d. h. Tumordurchmesser $<3 \mathrm{~cm}$, keine LK-Metastasen, keine extrahepatische Erkrankung, histologisch bestätigtes pCCA oder CA19-9> $100 \mathrm{kU} / \mathrm{L}$ mit Vorliegen radiologischer Zeichen einer malignen Stenose), wurde keine neoadjuvante Therapie durchgeführt. Dennoch wies diese Subgruppe eine 5-Jahres-Überlebensrate von $59 \%$ auf. Die übrigen 77 Patienten, die die Mayo-Kriterien nicht erfüllten, zeigten schlechte Ergebnisse mit einem 5-JahresGesamtüberleben < 20\% [140].

\subsubsection{Interventionelle Therapieverfahren}

\subsubsection{Perkutane Ablation}

\begin{tabular}{|l|l|l|}
\hline 4.28) & Konsensbasierte Empfehlung & Neu 2021 \\
\hline EK & $\begin{array}{l}\text { Lokale Verfahren (RFA/MWA) können nach Beschluss des } \\
\text { Tumorboards durchgeführt werden, falls keine Resektion } \\
\text { möglich ist. }\end{array}$ \\
\hline & starker Konsens \\
\hline
\end{tabular}

Grundsätzlich ist die thermische Ablation beim iCCA bis $3 \mathrm{~cm}$ Durchmesser möglich und klinisch effektiv [141-150]. Mit modernen, effektiveren Ablationstechniken und in Kombination mit selektiver Embolisation ist eine Ablation beim iCCA auch bis $5 \mathrm{~cm}$ Durchmesser in Analogie zum Vorgehen beim HCC möglich [151]. Die thermische Ablation wird in den EASL Guidelines mit einer Empfehlung C2 als „Kann-Option“ für „kleine Läsionen, die nicht chirurgisch zugänglich sind“ aufgeführt [152]. Es wird empfohlen, weitere klinische Studien durchzuführen. Auch in den NCCN Guidelines V3-2019 ist die thermische Ablation als Therapie des irresektablen iCCA explizit genannt [153].
Mehrere retrospektive Studien liegen vor, die den Wert der thermischen Ablation im historischen Vergleich mit akzeptablen Überlebensraten zeigen. In der bisher größten Single-CenterStudie mit 107 Patienten und 171 Tumoren [150] zeigte die Ablation bei primärem iCCA ein PFS nach 6, 12, 18 und 24 Monaten von $67,4 \% ; 41,5 \% ; 18,2 \%$ und $8,7 \%$ und ein OS nach 1,3 und 5 Jahren von 93,5\%, 39,6\% und 7,9\%. In einer Metaanalyse [143] betrugen die gepoolten 1-Jahres-, 3-Jahres- und 5-Jahres-Überlebensraten $82 \%$ (95\%-KI $72 \%$; $90 \%$ ), $47 \%$ (95\%-KI $28 \%$; $65 \%$ ) und $24 \%$ (95\%-KI 11\%; $40 \%)$.

\subsubsection{Intraarterielle Therapieverfahren}

\begin{tabular}{|l|l|l|}
\hline 4.29) & Konsensbasierte Empfehlung & Neu 2021 \\
\hline EK & $\begin{array}{l}\text { Beim fortgeschrittenen iCCA ohne extrahepatischen } \\
\text { Befall können intraarterielle Verfahren ab der Zweitlinie } \\
\text { oder additiv zur Chemotherapie, nach Vorstellung im } \\
\text { Tumorboard, erfolgen. }\end{array}$ \\
\hline & starker Konsens \\
\hline
\end{tabular}

Die arteriellen Verfahren sind in zahlreichen Studien evaluiert. Aufgrund der geringen Patientenzahl des seltenen Tumors gibt es bisher weder für eine primäre noch für eine sekundäre lokale Therapie randomisierte Studien, allerdings zahlreiche Kohortenstudien, Metaanalysen und systematische Reviews.

Als primäre Therapie werden selektive transarterielle Verfahren erwogen, falls bei Patienten eine Kontraindikation für eine systemische Chemotherapie vorliegt bzw. eine systemische Chemotherapie abgelehnt wird. Als sekundäre Therapie werden TACE, TARE und HAI allein bei Nichtansprechen oder kombiniert mit systemischer Therapie in Einzelfällen diskutiert. In den von Bridgewater publizierten „Guidelines for the diagnosis and management of intrahepatic cholangiocarcinoma, der International Liver Cancer Association (ILCA) aus dem Jahr 2014 [61] wurde folgende Einschätzung getroffen: TACE und TARE zeigen in Einzelfällen ein gutes Ansprechen mit vertretbarer Toxizität bei Patienten mit iCCA. Allerdings schließen die Autoren zum damaligen Zeitpunkt, dass aufgrund einer unzureichenden Studienlage noch keine allgemeine Empfehlung für diese Therapien ausgesprochen werden kann. Eine Phase-III-Studie zu dieser Fragestellung ist nach wie vor nicht berichtet worden, somit bleiben TACE und TARE derzeit Einzelfällen vorbehalten nach Besprechung im interdisziplinären Tumorbord.

Die lokoregionäre Therapie wird in mehreren Metaanalysen als wirksam hervorgehoben. Die TACE erreicht beim irresektablen iCCA allein ein medianes Gesamtüberleben von 12-17 Monaten und in Kombination mit systemischer Therapie einen zusätzlichen Überlebensvorteil von 2-12 Monaten [143, 145-148, 154]. Cuchetti et al. [149] konnten in einer Metaanalyse bei Patienten mit „mass-forming“ iCCA (OS: 19,9 Monate), bei TARE-naiven Patienten (OS: 24 Monate) und in Kombination mit systemischer Chemotherapie (OS: 19,5 Monate) die besten Überlebensraten 
erreichen. Solitäre Tumoren haben nach TARE ein höheres OS als multifokale Tumoren [150] (25 vs. 6,1 Monate [151]). Ähnliche Unterschiede zeigen sich zwischen gut und gering differenzierter Histologie [151] (18,6 vs. 9,7 Monate [152]).

Yang et al. fassen in einem systematischen Review 20 Arbeiten zusammen, allerdings konnte aufgrund der Datenheterogenität keine Metaanalyse durchgeführt werden. Dennoch zeigt diese Arbeit, dass transarterielle Verfahren sicher und effektiv sein können mit einem medianen Überleben von 12,4 Monaten nach TARE, interessanterweise trotz $33 \%$ der Patienten mit extrahepatischen Manifestationen [153]. Aufgrund der Daten einer gepoolten Analyse von 12 Studien mit einem medianen Überleben von 15,5 Monaten und einer Ansprechrate von $28 \%$ erwähnt die 2016 erschienene ESMO-Leitlinie [14] explizit auch die Möglichkeit einer TARE nach Versagen der Systemtherapie. In einer multizentrischen Auswertung [155] in 5 Krankenhäusern zeigte sich kein OS-Unterschied zwischen cTACE (13,4 Monate), DEB TACE (10,5 Monate), alleiniger Embolisation (TAE; 14,3 Monate) oder TARE (11,3 Monate) $(p=0,46)$. Vergleichbare Daten zum OS bei TACE und TARE haben auch Boehm et al. [146] in einer Metaanalyse berichtet, wobei darin die HAI zwar eine höhere Toxizität aber auch ein signifikant längeres OS aufwies.

Ein interessantes neues interventionell-radiologisches Konzept stellt die Chemosaturation mit einer Erhöhung der lokalen Dosis und Reduktion der Toxizität dar. Dieser Ansatz wird derzeit in Studien evaluiert und könnte in Zukunft einen Fortschritt für Patienten mit iCCA zeigen [156].

Sowohl mit TARE als auch mit HAI wurde in Studien [151, 157, 158] über Downstaging berichtet, das bei einigen Patienten eine R0-Resektion ermöglichte. [159]. Dies bestätigt die Notwendigkeit der erneuten Beurteilung der Patienten nach intraarteriellen Therapien in einem multidisziplinären Team bei gutem Ansprechen.

\subsubsection{Endoskopische Therapieverfahren}

\subsubsection{Präoperative biliäre Drainagen}

\begin{tabular}{|l|l|l|}
\hline 4.30) & Konsensbasierte Empfehlung & Neu 2021 \\
\hline EK & $\begin{array}{l}\text { Die Indikation für eine präoperative biliäre Drainage } \\
\text { sollte interdisziplinär getroffen werden. }\end{array}$ \\
\hline & starker Konsens, Klug entscheiden \\
\hline
\end{tabular}

\begin{tabular}{|l|l|l|}
\hline 4.31) & Konsensbasierte Empfehlung & Neu 2021 \\
\hline EK & $\begin{array}{l}\text { Bei Vorliegen einer Cholangitis sollte eine präoperative } \\
\text { biliäre Drainage umgehend erfolgen. }\end{array}$ \\
\hline & starker Konsens, Klug entscheiden \\
\hline
\end{tabular}

Die Indikation zur biliären Drainage stellt sich entsprechend dem Behandlungsziel, dabei kann eine präoperative biliäre Drainage
(PBD) bei kurativ intendierter Resektion oder eine palliative Drainage infrage kommen. Darüber hinaus hängt die Behandlungsstrategie von der Lokalisation des Gallenwegverschlusses (intrahepatisch, hilär, extrahepatisch) ab. Ein weiterer Aspekt ist der Zugangsweg: Die BD kann grundsätzlich perkutan-transhepatisch, transpapillär oder transgastrisch/transduodenal erfolgen.

Wenn eine kurativ intendierte Resektion möglich ist, ist diese die bevorzugte Behandlung für Patienten mit hilärem oder extrahepatischem Cholangiokarzinom. Etwa 25-35\% dieser Patienten sind Kandidaten für eine PBD beim hilären CCA. Die Frage der Indikationsstellung zu einer PBD ist allerdings nicht abschließend beantwortet. Die Evidenz ist beschränkt und fußt überwiegend auf retrospektiven Analysen und nur vereinzelt auf prospektiven, randomisierten Studien. Die Hyperbilirubinämie wurde in einer kürzlich veröffentlichte Single-Center-Studie als wichtigster modifizierbarer Risikofaktor für das negative, frühe postoperative Outcome des Patienten beobachtet [160]. Es werden dabei unterschiedliche cut-off levels (orientierend an unterschiedlichen Resektionsausmaßen) angegeben, eine ausreichende Evidenz für einen entsprechenden Wert gibt es nicht. Andererseits kann eine PBD selbst das frühe postoperative Outcome des Patienten negativ beeinflussen, bspw. durch Komplikationen der interventionellen Therapie. Es wird zudem eine erhöhte Morbidität auch bei erfolgreicher Drainage gefunden und bspw. eine Ursache im möglichen Einschleppen von Keimen in das Gallenwegsystem durch die interventionelle Therapie vermutet. Unstrittig ist aber die Notwendigkeit einer PBD, wenn eine Cholangitis primär vorliegt.

Die PBD muss für das intrahepatische Gallengangkarzinom und das perihiläre Gallengangkarzinom getrennt betrachtet werden. Intrahepatische Gallengangkarzinome gehen nur selten mit einer Kompression der zentralen Gallenwegstrukturen und einem Ikterus einher. Eine präoperative Gallengangdekompression ist in diesen Fällen daher nur vor ausgedehnten Resektionen und Beteiligung der Gallenwege des „Future Liver Remnant“ erforderlich. Auch die Frage nach dem technischen Vorgehen bei der präoperativen Drainage ist nicht abschließend geklärt. Abgesehen von der lokalen Expertise im endoskopischen oder perkutanen Vorgehen, liegen auch im Studiensetting widersprüchliche Ergebnisse vor. Dies betrifft sowohl das Auftreten von Komplikationen durch die jeweilige Prozedur als auch die Erfolgsraten, wobei diese bei der PTCD etwas höher zu sein scheinen.

Es liegen 2 Metaanalysen mit bis zu 4 retrospektiven, nicht randomisierten Studien vor [161, 162]. In diesen wird eine vergleichbare bzw. etwas höhere Morbidität nach ERCP im Vergleich zur PTCD gefunden. Eine randomisierte Studie (Einschlusskriterien: Bilirubin $>2,9 \mathrm{mg} / \mathrm{dl}$, geplante erweiterte Leberresektion) wurde vorzeitig beendet, da in der PTCD-Gruppe eine signifikant höhere (Gesamt-)Mortalität (41\% von 27 Patienten) als in der endoskopisch gelegten Drainage-Gruppe (11\% von 27 Patienten) $(p=0,03)$ vorlag. Allerdings war bei $56 \%$ der Patienten zusätzlich zur endoskopisch gelegten Drainage eine perkutan gelegte Drainage erforderlich. Zudem entwickelten 16 (59\%) Patienten nach PTCD eine Cholangitis vs. 10 (37\%) nach ERCP [163]. 
Wahrscheinlich hat auch die jeweilige Expertise im Zentrum einen Einfluss auf das Outcome bei PBD. In einer multizentrischen, retrospektiven Analyse aus China zeigte sich im Vergleich einer ERCP vs. PTCD eine höhere periinterventionelle Morbidität in der ERCP-Gruppe [164]: Nach ERCP hatten $37 \%$ der Patienten eine Cholangitis und $17 \%$ eine Pankreatitis gegenüber von $22 \%$ mit Cholangitis und $2 \%$ mit Pankreatitis nach PTCD-Anlage.

Die in Japan bevorzugt gelegte nasobiliäre Drainage erscheint in westlichen Ländern wenig praktikabel [165], zudem scheint sie keinen Vorteil gegenüber einer Plastikstentanlage zu bieten [166].

Das Risiko einer Tumorzellverschleppung durch den Zugangsweg der Drainage ist zwar selten, wird nach PTCD aber beobachtet - nicht aber nach endoskopischer Therapie [167, 168].

Vor Augmentationsverfahren der Leber (z. B. Pfortaderembolisation) sollte zumindest eine biliäre Drainage des zukünftigen Restlebergewebes (Future Liver Remnant) erfolgen [169]. Die Drainage des zu entfernenden Lebergewebes hat nur einen geringen Einfluss auf die Hypertrophieinduktion [170].

Zusammenfassend sollte die Indikation zu einer PBD in einem hepatobiliären Zentrum erfolgen; dort ist eine interdisziplinäre Planung der Behandlung unter Einschluss des chirurgischen, des interventionellen/endoskopischen und radiologischen Behandlers möglich. Die interventionelle Therapie muss dabei auch durch alternative Drainagewege möglich sein, da eine primär insuffiziente Drainage nicht selten ist und dann eine alternative Therapiestrategie ergriffen werden muss.

Ergänzende Literaturübersicht s. Anhang ( $\triangleright$ Tab. 10: Übersicht über Literatur zur präoperativen biliären Drainage (PBD), S. 218)

\subsubsection{Palliative biliäre Drainage}

\begin{tabular}{|l|l|l|}
\hline 4.32) & Konsensbasierte Empfehlung & Neu 2021 \\
\hline EK & $\begin{array}{l}\text { Eine palliative biliäre Drainage soll Patienten mit symp- } \\
\text { tomatischem Gallenwegverschluss angeboten werden. }\end{array}$ \\
\hline & starker Konsens \\
\hline
\end{tabular}

\begin{tabular}{|l|l|l|}
\hline $\mathbf{4 . 3 3}$ & Konsensbasierte Empfehlung & Neu 2021 \\
\hline EK & $\begin{array}{l}\text { Eine palliative Drainage sollte in einem erfahrenen } \\
\text { Zentrum durchgeführt werden, da dort auch alternative } \\
\text { Drainageverfahren zur Verfügung stehen. }\end{array}$ \\
\hline & starker Konsens \\
\hline
\end{tabular}

Die interventionelle Drainage des Gallenwegsystems bei malignem Gallenwegverschluss ermöglicht eine Symptomverbesserung, eine Verbesserung der Leberfunktion und die Behandlung einer Cholangitis. Mit einem dieser Therapieziele ist eine palliative biliäre Drainage bei biliären Karzinomen angezeigt. Die interventionelle Vorgehensweise ist dabei abhängig von der Lokalisation des Verschlusses bzw. dem vorliegenden biliären Tumor (intrahepatisches vs. perihiläres vs. distales Cholangiokarzinom). Das
Vorgehen wird darüber hinaus von der verfügbaren Technik mitbeeinflusst (ERCP, PTCD, EUS-gesteuertes biliäres Drainageverfahren). Eine detaillierte präinterventionelle Behandlungsplanung auf der Basis aussagekräftiger Bildbefunde (in der Regel MRCP) ist dabei unabdingbar [171]. Die Behandlungsintention sollte vor der Intervention definiert und dokumentiert werden und muss das Ziel einer kompletten vs. inkompletten Drainage einschließen.

Grundsätzlich kann die Lebensqualität bei Hyperbilirubinämie durch eine erfolgreiche Drainage verbessert werden [172]. Für ein verlängertes Überleben durch eine erfolgreiche Drainage in der Palliation gibt es zahlreiche Hinweise [173]. Es wird eine 30-Tages-Mortalität bei der distalen biliären Stenose von 2-20\% und von 9-20\% in der perihilären Stenose berichtet [174-177].

Randomisierte Studien fokussieren überwiegend auf den distalen, periampullären malignen Gallenwegverschluss [178, 179]. Bei ihm kann eine primär retrograde endoskopische Drainage in der Regel empfohlen werden. Ein selbstexpandierender Metallstent (SEMS) sollte als primäre Drainageoption gewählt werden und scheint einer Plastikendoprothese überlegen [180]. Bei palliativer Indikation kann ein ummantelter, entfernbarer oder nicht ummantelter SEMS eingesetzt werden. Die Durchführung einer EUS-gesteuerten Drainage kann dann einer PTCD nach frustranem ERCP-Versuch überlegen sein [181, 182]. Erste randomisierte Studien sehen bereits im primären Drainageversuch die EUSgesteuerte Technik der ERCP ebenbürtig [183, 184].

Beim perihilärem, symptomatischem Gallenwegverschluss werden PTCD oder ERCP mit unilateraler oder bi-/trilateraler Drainage eingesetzt. Eine EUS-gesteuerte Drainage kommt nur in Ausnahmefällen (z. B. transgastrische Drainage der linken Leber) infrage. Eine Drainage von mindestens $50 \%$ des (mutmaßlich funktionalen) Leberanteils wird empfohlen, wobei eine Kontrastierung von nicht drainierten Gangsegmenten vermieden werden sollte [185].

Randomisierte Studien berichten von höherem Erfolg und niedrigeren Komplikationen der PTCD vs. ERCP beim perihilären Gallenwegverschluss [173, 174, 186, 187], aber die Lebensqualität könnte bei dem inneren Drainageverfahren größer sein [188].

Einige frühere randomisierte Daten sprechen für eine unilaterale Drainage, indem auch nur dieser Gangabschnitt dargestellt und intubiert wird $[171,189]$. Um eine Cholangitis durch abgehängte Gangareale zu vermeiden, ist allerdings eine effektive Drainage aller dargestellten Gänge hilfreich [190, 191]. Das weist auch auf die für die klinische Situation unzureichende Vereinfachung bei Studien und der einzelnen Patientenbehandlung hin, die im uni- vs. bilateralen Drainageansatz zu sehen ist. Da das Gallenwegsystem zumindest 3 Doppelsegmente umfasst, kann eine komplette Drainage in fortgeschrittenen perihilären Obstruktionen erst mit einem Dreifach-Stenting erreicht werden. Ein univs. bilateraler Ansatz in Studien sollte daher zukünftig für die Zielbestimmung einer kompletten vs. inkompletten Drainage verlassen werden. Dies wird in der Studienkonzeption wie auch in der Behandlungsplanung in der Klinik nicht immer berücksichtigt. Randomisierte Studien konnten die effektive Drainage mittels in der Regel bilateralen - SEMS zeigen [173, 181, 192]. Dabei 
scheint eine ,SEMS-neben-SEMS-“ einer ,SEMS-in-SEMS'-Technik gleichgestellt zu sein [181]. Es kommen nur nicht ummantelte, damit nicht entfernbare SEMS infrage, damit Seitenäste durch die Maschen des Stents drainiert werden können. Eine komplette Drainage muss als Ziel erreichbar sein. Das Outcome im weiteren Verlauf nach SEMS-Einlage im Vergleich zu Plastikstents ist nicht belegt. Eine Reintervention bei Komplikationen oder erneuter Symptomatik scheint nach Implantation von permanenten SEMS erschwert. Eine primäre Therapie mit multisegmentaler Plastikstenteinlage kann beim perihilären biliären Verschluss damit auch weiterhin als vorrangiger Therapieansatz gesehen werden.

Die Drainage eines Gangabschnitts, bei dem bereits eine Atrophie der abhängigen Lebersegmente eingetreten ist, sollte nicht erfolgen, da erhöhte Komplikationsraten und keine wesentliche Verbesserung des Patienten-Outcomes zu erwarten sind [193].

Da ein Drainageversuch mit einer Technik - bspw. der ERCP frustran verlaufen kann, sollte zumindest eine alternative Technik - bspw. PTCD und/oder EUS-gesteuerte biliäre Drainage - verfügbar sein. Die Häufigkeit, mit der eine biliäre Drainage im Zentrum vorgenommen wird, scheint einen wesentlichen Einfluss auf den Erfolg des Eingriffs und das Outcome beim Patienten zu haben $[176,194]$. Beides spricht für eine Behandlung dieser Patienten im erfahrenen Zentrum.

Literaturübersicht s. Anhang ( $\triangleright$ Tab. 11: Übersicht über Literatur zur biliären Drainage (BD) bei Cholangiokarzinom. S. 219)

\subsubsection{Intraduktale lokoregionäre Therapieverfahren}

\begin{tabular}{|l|l|l|}
\hline 4.34) & Konsensbasierte Empfehlung & Neu 2021 \\
\hline EK & $\begin{array}{l}\text { Intraduktale, lokal ablative Verfahren (Photodynamische } \\
\text { Therapie und intraduktale RFA) können nach Beschluss } \\
\text { des Tumorboards durchgeführt werden, um eine effek- } \\
\text { tive Palliation zu ermöglichen. }\end{array}$ \\
\hline & starker Konsens \\
\hline
\end{tabular}

Intraduktale, lokal ablative Verfahren können bei einem lokal begrenzten Tumor in Betracht gezogen werden. Es handelt sich dabei um eine palliative Behandlungsform. Für eine OutcomeVerbesserung mit einer lokal ablativen Therapie bei einem metastasierten Tumor finden sich keine Belege. Es stehen die Photodynamische Therapie (PDT) und die intraduktale Radiofrequenzablation (iRFA) zur Verfügung, wobei Letztere sich von der perkutanen RFA wesentlich unterscheidet. Die lokal ablativen Therapien werden in aller Regel mit einer endoskopischen Stenttherapie kombiniert und sind nur in dieser Kombination durch Studien geprüft. Dadurch ist eine lokale Tumordestruktion über wenige Millimeter zu erreichen. Die iRFA wird nach Heranführen einer bipolaren Sonde mittels ERCP in den tumorös stenosierten Gallenwegabschnitt durch Anwendung von hochfrequentem Wechselstrom durchgeführt [195]. Mit der PDT wird ebenfalls eine lokale Tumordestruktion über wenige Millimeter Eindringtiefe erreicht. Dafür muss allerdings einige Zeit vor einer lokalen Lichtbestrah- lung des Tumorareals in einer ERCP ein Photosensitizer intravenös appliziert werden, der die Tumorzellen besonders lichtempfindlich werden lässt und diese sowie Zellen der Neovaskularisation zerstört [196]. Bei der PDT werden unterschiedliche Photosensitizer eingesetzt. Porfimer-Natrium (Photofrin) ist der am häufigsten genutzte Sensitizer, Temoporfin (Foscan) und Dihematoporphin Ether (Photosan-3) sind weitere Produkte. Photofrin ist zugelassen für die Behandlung von Patienten mit nicht resezierbarem perihilärem Cholangiokarzinom. 5-Aminolävulinsäure scheint beim Gallenwegkarzinom nicht wirksam [197]. Vergleiche einer Effektivität der Sensitizer liegen nicht vor.

Eine randomisierte Studie zeigte ein verbessertes Überleben im Vergleich zu einer alleinigen Stenttherapie für die iRFA [198] und 2 randomisierte Studien einen Überlebensvorteil für die PDT [199, 200]. Eine randomisierte Studie zeigte ein schlechteres Outcome für die PDT im Vergleich zum Stent bei Patienten mit lokal fortgeschrittenen und metastasierten perihilären Tumoren [201]. In einer gepoolten Analyse wird ein Überleben von 413 Tage vs. 183 Tage für die PDT gegenüber der alleinigen Stenttherapie gefunden [196]. In der Kombination mit einer systemischen Chemotherapie zeigte sich die PDT der PDT + Stent-Gruppe und der „Stent-allein“-Gruppe in retrospektiver Analyse überlegen [202, 203].

Die Auswahl einer PDT gegenüber einer IRFA ist durch direkte Vergleichsstudien nicht abgesichert [204, 205]. Die IRFA scheint technisch einfacher und mit hoher primärer Erfolgsrate verbunden; ob die Komplikationsrate der iRFA gegenüber der PDT vergleichbar ist, ist nicht klar [195]. Für die PDT sprechen die breitere Erfahrung und die größere Zahl an in Studien eingeschlossenen Patienten [206] sowie retrospektive Daten für die Kombination mit der systemischen Chemotherapie.

Literaturübersicht s. Anhang ( $\vee$ Tab. 12: Übersicht über Literatur zu intraduktalen, lokal ablativen Verfahren (PBD). S. 220)

\subsubsection{Stereotaxie}

\begin{tabular}{|l|l|}
\hline $\mathbf{4 . 3 5})$ & Konsensbasierte Empfehlung \\
\hline EK & $\begin{array}{l}\text { Eine Hochpräzisionsradiotherapie (Stereotactic Body } \\
\text { Radiotherapy; SBRT) kann nach Beschluss in einer } \\
\text { Tumorkonferenz bei fehlenden alternativen Therapie- } \\
\text { optionen angeboten werden. }\end{array}$ \\
\hline & starker Konsens \\
\hline
\end{tabular}

Eine Reihe von Autoren hat in den vergangenen Jahren berichtet, dass nach einer definitiven Radiotherapie die Höhe der Dosis mit einer hohen Lokalkontrollrate und auch mit dem Überleben der Patienten korreliert [207-209]. Obwohl das zunächst mit einer konventionell fraktionierten Radio(chemo)therapie beobachtet wurde [207, 209], hat die Mehrzahl der Studien der letzten Jahre eine SBRT dafür eingesetzt, die typischerweise eine Dosiseskalation im Zentrum der Bestrahlungsvolumina verwendet [208, 210, 211]. Die Dosisabhängigkeit gilt sowohl für intrahepatische als 
auch für perihiläre CCA. Darüber hinaus ist eine sequenzielle Chemotherapie vor und/oder nach der Radiotherapie ein weiterer Faktor für die Verlängerung des Überlebens, und einige Studien haben eine Kombination der Radiotherapie mit einer sequenziellen Chemotherapie durchgeführt [209, 210].

Die Erfahrungen mit SBRT zur Behandlung von Cholangiokarzinomen sind begrenzt. Eine Metaanalyse fasst 226 Patienten in 4 prospektiven [212-215] und 7 retrospektiven Studien zusammen [210]. Die gepoolte 1-Jahres-Lokalkontrollrate war 81,8\% (95\%-KI 69,4; 89,9\%), wenn die 2-Gy-Äquivalenzdosis (EQD2) $\geq 71,3$ Gy war und darunter bei 74,7\% (95\%-KI 57,1\%; 86,7\%). Das mediane Überleben lag bei 13,6 Monaten (10-35,5 Monate). Die berichteten Toxizitäten waren moderat mit $<10 \%$ Akuttoxizität $\geq 3$ und 10-20\% Spättoxizität, v. a. als duodenale und gastrale Ulzera.

Die Protonenstrahlbestrahlung ist eine weitere Methode zur Anwendung einer hochdosierten Radiotherapie. Die meisten Daten beschränken sich auf retrospektive Studien mit nur einer Institution. Eine prospektive, multi-institutionelle Phase-II-Studie untersuchte die Wirksamkeit und Sicherheit einer hochdosierten hypofraktionierten Protonentherapie bei intrahepatischen Cholangiokarzinomen ( $n=37$; daneben auch bei 44 Patienten mit HCC). Eine Dosis von 67,5-Gray-Äquivalenten wurde in 15 Fraktionen angewendet. Die 2-Jahres-Lokalkontroll- und Gesamtüberlebensrate betrug in der Cholangiokarzinom-Kohorte 94,1\% bzw. $46,5 \%$ [216].

Zur interstitiellen Brachytherapie von Cholangiokarzinomen ist die Evidenzlage niedrig. In einer monozentrischen, retrospektiven Serie wurden 15 Patienten mit histologisch gesicherten Tumoren an insgesamt 27 Läsionen mit einer interstitiellen Brachytherapie behandelt. Die mediane Dauer der lokalen Tumorkontrolle betrug 10 Monate und die mediane Überlebenszeit 14 Monate nach der Behandlung [217].

\subsubsection{Nachsorge}

\begin{tabular}{|l|l|}
\hline 4.36) & Konsensbasierte Empfehlung \\
\hline EK & $\begin{array}{l}\text { Nach Resektion/Ablation eines CCA sollte nach 4- } \\
12 \text { Wochen erstmals, im ersten Jahr alle 3 Monate und im } \\
\text { zweiten Jahr alle 3-6 Monate ein biphasisches CT oder } \\
\text { ein dynamisches MRT durchgeführt werden. }\end{array}$ \\
\hline & starker Konsens \\
\hline
\end{tabular}

Es liegt keine Evidenz hinsichtlich des genauen Vorgehens bei der Nachsorge vor. Beim praktischen Vorgehen wird nach einer Resektion und Ablation eines Cholangiokarzinoms nach 4-12 Wochen ein biphasisches CT oder dynamisches MRT empfohlen. Im weiteren ersten Jahr alle 3 Monate, im zweiten Jahr alle 3-6 Monate. Die Nachsorge sollte für insgesamt 5 Jahre durchgeführt werden und ab dem dritten Jahr eine jährliche Schnittbildgebung umfassen. Dieses Vorgehen erfolgt analog der NCCN Guideline 2019 [142].

\subsection{Systemtherapie}

\subsubsection{Adjuvante Therapie}

\begin{tabular}{|l|l|l|}
\hline 4.37) & Konsensbasiertes Statement & Neu 2021 \\
\hline EK & $\begin{array}{l}\text { Nach chirurgischer Tumorentfernung besteht ein } \\
\text { Rezidivrisiko von } 40-80 \% \text { Insbesondere Patienten mit } \\
\text { Lymphknotenmetastasen (N1), positiven Resektionsrän- } \\
\text { dern (R1) oder niedrigem Differenzierungsgrad (G3) } \\
\text { haben ein hohes Rezidivrisiko. }\end{array}$ \\
\hline & starker Konsens \\
\hline
\end{tabular}

\begin{tabular}{|l|l|}
\hline $\mathbf{4 . 3 8}$ & Evidenzbasierte Empfehlung \\
\hline $\begin{array}{l}\text { Empfeh- } \\
\text { lungsgrad } \\
\mathbf{B}\end{array}$ & $\begin{array}{l}\text { Aufgrund des hohen Rezidivrisikos sollte Patienten } \\
\text { nach chirurgischer Tumorentfernung (R0, R1) eine } \\
\text { adjuvante Systemtherapie mit Capecitabin } \\
\text { angeboten werden. }\end{array}$ \\
\hline $\begin{array}{l}\text { Level of } \\
\text { Evidence } \\
\mathbf{2}\end{array}$ & Primärrecherche: Primrose 2019 [108] \\
\hline & starker Konsens \\
\hline
\end{tabular}

Aufgrund des subendothelialen Wachstums entlang der Perineuralscheiden beträgt das Rezidivrisiko nach radikaler chirurgischer Tumorentfernung für ein perihiläres Cholangiokarzinom (CCA) 40$80 \%$ (Auflistung der Studienergebnisse siehe [218]). Auch für andere Lokalisationen des CCA und für Gallenblasenkarzinome (GB-CA) ist das Rezidivrisiko ähnlich hoch. Risikofaktoren für ein frühes Rezidiv sind vor allem Lymphknotenmetastasen (N1), R1-Status und ein niedriger Differenzierungsgrad (G3). Eine Metaanalyse retrospektiver und einarmiger Studien mit mehr als 6000 Patienten mit CCA oder GB-CA ergab für Patienten mit einem dieser Risikofaktoren einen Vorteil für eine adjuvante Therapie [219]. Zusätzlich liegen mit den Ergebnissen der BILCAP-Studie jetzt auch erstmals Daten einer randomisierten Phase-III-Studie vor, die den Nutzen einer adjuvanten Chemotherapie zeigen [108]. In dieser Studie wurde bei 447 Patienten mit CCA oder muskelinvasivem GB-CA sowie vollständiger makroskopischer Tumorentfernung (R0 oder R1) die Gabe von Capecitabin $\left(1250 \mathrm{mg} / \mathrm{m}^{2}\right.$ zweimal täglich an Tag 1-14 bei einer Zyklusdauer von 21 Tagen, insgesamt 8 Zyklen) mit der alleinigen Nachsorge verglichen. Der Therapiebeginn sollte innerhalb von 12 Wochen postoperativ bei Patienten mit ECOG 0-1 erfolgen, erlaubt war eine Ausdehnung des Zeitraums bis auf 16 Wochen.

In der Per-Protokoll-Analyse ergab sich ein signifikanter Unterschied im medianen Gesamtüberleben von 53 versus 36 Monaten (HR: 0,75; $95 \%$-KI 0,58; 0,97, p = 0,028). In der Intention-to-TreatAnalyse war der Unterschied zwischen beiden Gruppen vergleichbar mit 51 versus 36 Monate (HR: 0,81; $95 \%-K I$ 0,63; 1,04, $p=0,097)$, allerdings nicht signifikant. Die mediane Zeit von der OP bis zum Therapiebeginn lag bei 10,3 Wochen. Die französische 
PRODIGE-12-Studie konnte ebenfalls durch Gemcitabin und Oxaliplatin im Vergleich zur alleinigen Nachsorge keine signifikante Verbesserung des Gesamtüberlebens erzielen [220]. Zurzeit wird in der internationalen ACTICCA-1-Studie unter Beteiligung fast aller universitären Zentren in Deutschland die Wirksamkeit einer adjuvanten Chemotherapie mit Gemcitabin und Cisplatin gegen Capecitabin getestet [221].

Auf der Basis der zitierten Metaanalyse und der BILCAP-Daten sollte allen Patienten und insbesondere denjenigen mit einem der genannten Risikofaktoren (N1, R1 oder G3) eine adjuvante Therapie mit Capecitabin oder ein Einschluss in die ACTICCA-1-Studie angeboten werden. Aufgrund fehlender Evidenz besteht außerhalb von klinischen Studien zurzeit keine Indikation für eine adjuvante Strahlentherapie.

\subsubsection{Neoadjuvante Therapie primär resektabler Tumoren}

\begin{tabular}{|l|l|l|}
\hline 4.39) & Konsensbasierte Empfehlung & Neu 2021 \\
\hline EK & $\begin{array}{l}\text { Eine neoadjuvante Chemotherapie soll bei primär } \\
\text { resektablen Tumoren nicht außerhalb von klinischen } \\
\text { Studien erfolgen. }\end{array}$ \\
\hline & starker Konsens \\
\hline
\end{tabular}

\subsubsection{Systemtherapie lokal fortgeschrittener Tumoren}

\begin{tabular}{|l|l|l|}
\hline $\mathbf{4 . 4 0 )}$ & Konsensbasierte Empfehlung & Neu 2021 \\
\hline EK & $\begin{array}{l}\text { Bei primär irresektablen Tumoren sollte unter einer } \\
\text { Tumortherapie eine erneute Vorstellung im Tumorboard } \\
\text { mit der Frage einer sekundären Resektabilität erfolgen. }\end{array}$ \\
\hline & starker Konsens \\
\hline
\end{tabular}

Bei Patienten mit grenzwertig resektablen Tumoren kann als individuelles Konzept eine Chemotherapie mit kurzfristiger erneuter Evaluation der Operabilität durchgeführt werden. Dabei liegen weder Daten zu einer standardisierten Definition der Resektabilität noch zur systemischen palliativen Therapie vor. Die Chemotherapie sollte in Analogie zur palliativen Chemotherapie erfolgen. In der Phase-III-Studie von Valle et al. lagen allerdings die objektiven Ansprechraten für Gallenwegkarzinome bei lediglich 19\% und für Gallenblasenkarzinome bei 38\% [222]. In einer neueren einarmigen Phase-II-Studie mit 60 inoperablen oder metastasierten Patienten (39 mit intrahepatischem, 9 mit extrahepatischem CCA und 13 mit GB-CA) wurde das Ansprechen auf eine Dreifachtherapie mit Gemcitabin, Cisplatin und nab-Paclitaxel untersucht [223]. $45 \%$ der Patienten erreichten eine partielle Remission und 12 Patienten konnten einer sekundären Operation zugeführt werden. Daher kann in ausgewählten Einzelfällen eine intensivierte Chemotherapie bei lokal fortgeschrittenen Tumoren mit dem Ziel einer sekundären Operation durchgeführt werden.
Prinzipiell gilt jedoch auch für alle anderen eingesetzten Systemtherapien, z. B. in klinischen Studien, dass bei gutem Ansprechen die Resektabilität nach 2-3 Monaten erneut evaluiert werden sollte.

\subsubsection{Palliative Systemtherapie}

\begin{tabular}{|c|c|c|}
\hline 4.41) & Evidenzbasierte Empfehlung & Neu 2021 \\
\hline $\begin{array}{l}\text { Empfeh- } \\
\text { lungsgrad } \\
\text { A }\end{array}$ & \multicolumn{2}{|c|}{$\begin{array}{l}\text { Allen Patienten mit Cholangio- oder Gallenblasen- } \\
\text { karzinom soll bei adäquatem Allgemeinzustand in } \\
\text { der inoperablen lokal fortgeschrittenen oder } \\
\text { metastasierten Situation eine palliative Systemthe- } \\
\text { rapie angeboten werden. }\end{array}$} \\
\hline \multirow{2}{*}{$\begin{array}{l}\text { Level of } \\
\text { Evidence } \\
\mathbf{2}\end{array}$} & \multicolumn{2}{|c|}{$\begin{array}{l}\text { Primärrecherche: Valle } 2010 \text { [222], Okusaka } 2010 \\
\text { [224], Valle } 2014 \text { [225], Park } 2015 \text { [226] }\end{array}$} \\
\hline & Konsens & \\
\hline
\end{tabular}

Bei der Indikationsstellung zur Chemotherapie sind der Allgemeinzustand des Patienten, die Komorbiditäten, die Patientenpräferenzen sowie die Toxizität der geplanten Schemata zu berücksichtigen [61, 227]. In der ABC-02-Studie (s. u.) zeigte sich ein Überlebensvorteil für alle Tumorlokalisationen. Der Vorteil war am deutlichsten für Patienten mit einem ECOG-Performance-Status (PS) 0 oder 1.

\subsubsection{Erstlinientherapie}

\begin{tabular}{|l|l|}
\hline $\mathbf{4 . 4 2 )}$ & Evidenzbasierte Empfehlung \\
\hline $\begin{array}{l}\text { Empfeh- } \\
\text { lungsgrad }\end{array}$ & $\begin{array}{l}\text { Als Systemtherapie soll in der Erstlinie die } \\
\text { Kombination Gemcitabin und Cisplatin } \\
\text { angeboten werden. }\end{array}$ \\
\hline $\begin{array}{l}\text { Level of } \\
\text { Evidence } \\
\mathbf{2}\end{array}$ & $\begin{array}{l}\text { Primärrecherche: Valle 2010 [222], Okusaka } 2010 \\
\text { [224], Valle 2014 [225], Park 2015 [226] }\end{array}$ \\
\hline & Konsens \\
\hline
\end{tabular}

Zwei Studien, die britische ABC-02-Studie [222] und die japanische BT22-Studie [224], haben in einer gemeinsamen Auswertung bei insgesamt fast 500 Patienten die Überlegenheit einer Kombinationstherapie bestehend aus Gemcitabin und Cisplatin gegenüber einer Monotherapie mit Gemcitabin gezeigt. In der im Vorfeld geplanten gemeinsamen Auswertung beider Studien [225] konnte das mediane Gesamtüberleben von 8,0 auf 11,6 Monate $(H R=0,65,95 \%-K I 0,54 ; 0,78, p<0,001)$ gesteigert werden. Die beiden Studien führten auch separat zu einer signifikanten Verlängerung des medianen Gesamtüberlebens im Therapiearm mit Gemcitabin und Cisplatin. Dabei ist besonders zu berücksichtigen, dass mit der Applikation von Gemcitabin $1000 \mathrm{mg} / \mathrm{m}^{2}$ und Cisplatin $25 \mathrm{mg} / \mathrm{m}^{2}$ an den Tagen 1 und 8 bei einer Zyklusdauer von 21 Tagen ein gut verträgliches Therapieschema implementiert wurde. So zeigten sich keine Unterschiede 
in beiden Therapiegruppen in Bezug auf das Auftreten von schwergradigen (CTCAE Grad 3 und 4) Nierenfunktionsstörungen (Gemcitabin und Cisplatin versus Gemcitabin: $1,5 \%$ vs. $1 \%$, $p=0,83)$ sowie von Übelkeit $(4,0 \%$ versus $3,5 \%, p=0,78)$ und Erbrechen (5,1 vs. 5,5\%, $p=0,65)$. Eine Metaanalyse aus 2015 bestätigte Gemcitabin und Cisplatin als wirksame Therapie bei diesen Tumoren [226].

Bei Patienten mit ECOG 2 kann alternativ eine Monotherapie mit Gemcitabin erfolgen, und bei eingeschränkter Nierenfunktion kann Oxaliplatin statt Cisplatin eingesetzt werden [227]. Die Lebenserwartung bei symptomorientierter Therapie beträgt nach historischen Daten dagegen nur ca. 2,5 bis 4,5 Monate [225].

Alternativ zu dieser Erstlinientherapie wird die Teilnahme an klinischen Studien empfohlen.

\subsubsection{Therapie nach Versagen der Erstlinientherapie}

\begin{tabular}{|l|l|l|}
\hline $\mathbf{4 . 4 3}$ & Konsensbasierte Empfehlung & Neu 2021 \\
\hline EK & $\begin{array}{l}\text { Nach Versagen oder Unverträglichkeit der Erstlinienthe- } \\
\text { rapie soll Patienten mit adäquatem Allgemeinzustand } \\
\text { eine weitere Therapie angeboten werden. }\end{array}$ \\
\hline & Konsens \\
\hline
\end{tabular}

\begin{tabular}{|l|l|l|}
\hline $\mathbf{4 . 4 4 )}$ & Konsensbasierte Empfehlung & Neu 2021 \\
\hline EK & $\begin{array}{l}\text { Als medikamentöse Zweitlinientherapie kann eine } \\
\text { Therapie mit FOLFOX angeboten werden. }\end{array}$ \\
\hline & Konsens \\
\hline
\end{tabular}

\begin{tabular}{|c|c|c|}
\hline 4.45) & Konsensbasierte Empfehlung & Neu 2021 \\
\hline EK & \multicolumn{2}{|c|}{$\begin{array}{l}\text { In einer palliativen Situation bei Patienten mit ECOG 0-1 } \\
\text { sollten eine molekulare Charakterisierung des Tumors } \\
\text { und eine Vorstellung in einem Interdisziplinären/ } \\
\text { Molekularen Tumorboard erfolgen. }\end{array}$} \\
\hline & Konsens, Klug entscheiden & \\
\hline
\end{tabular}

Für den Nutzen einer Zweitlinientherapie bei radiologisch progredienter Tumorerkrankung unter der Erstlinientherapie liegen Daten aus der ABC-06-Studie vor [228], die jedoch noch nicht als vollständige Publikation vorgelegt worden sind. Zusätzlich erfolgte im April 2021 die Zulassung für Pemigatinib für Tumoren mit einer Fibroblasten-Wachstumsfaktor-Rezeptor-2(FGFR2)-Fusion oder einem FGFR2-Rearrangement, die nach mindestens einer Systemtherapie progredient sind (s. u.).

In der randomisierten Phase-III-Studie ABC-06 wurde ein modifiziertes FOLFOX-Regime gegen eine alleinige symptomorientierte Therapie verglichen und eine moderate Verbesserung des medianen Gesamtüberlebens von 5,3 Monate auf 6,2 Monate mit Chemotherapie erreicht (HR: 0,69 (95\%-KI 0,50; 0,97; $p=0,032)$ ) [228]. Grundsätzlich wurde der mögliche Nutzen einer Zweitlinientherapie im Vergleich zu einer alleinigen symptomorientierten Therapie auch in einer Metaanalyse mit mehr als 700 Patienten gezeigt [229]. Für Patienten mit Zweitlinientherapie betrug das mediane Gesamtüberleben 7 Monate bei einer Ansprechrate von $8 \%$. In einer retrospektiven kanadischen Fallserie erhielten von 378 Patienten allerdings nur $25 \%$ eine Zweitlinientherapie. Als positive prognostische Faktoren ergaben sich in dieser Auswertung eine Kombinationschemotherapie sowie ein ECOG PS 0-1 [230]. Aus weiteren retrospektiven Fallserien ergibt sich auch ein Signal für eine Monotherapie mit 5-Fluorouracil oder Capecitabin sowie einer Kombination dieser Substanzen mit Irinotecan oder Oxaliplatin [231]. Zusammengefasst zeigen jedoch alle diese Ergebnisse nur einen geringen Vorteil einer Systemtherapie für das Gesamtüberleben, sodass möglichst über klinische Studien dringend neue Therapieoptionen evaluiert werden sollten.

Die Zulassung von Pemigatinib beruht auf der einarmigen Phase-II-Studie FIGHT-202 [232]. Als primärer Endpunkt zeigten 38 von 107 Patienten (36\%) ein Ansprechen auf die Tumortherapie, davon 35 Patienten mit einer partiellen und 3 Patienten mit einer kompletten Remission. Die mediane Dauer des Therapieansprechens war 7,5 Monate (95\%-KI 5,7; 14,5) [232].

Als Beispiel zeigen die Daten zu Tumoren mit FGFR2-Fusionsgenen, dass molekulargenetische Marker eine immer größere Rolle für die Therapieauswahl spielen. Die molekulare Charakterisierung von Cholangiokarzinomen deutet darauf hin, dass diese Tumorentität sich in besonderem Maße für eine molekular gerichtete Therapie eignet [233] und dass diese Patienten bei gutem Allgemeinzustand (ECOG 0-1) nach einem Versagen von verfügbaren Therapien in einem molekularen Tumorboard vorgestellt werden sollten. Beispiele für molekulare Veränderungen, die therapeutisch genutzt werden können, sind neben FGFR2 insbesondere die Untersuchung auf Mikrosatelliteninstabilität, NTRK-Fusionsgene, Amplifikationen von HER2, die BRAF-V600E-Mutation oder Mutationen im IDH1-Gen.

- Die Bestimmung auf Mikrosatelliteninstabilität soll Tumoren mit funktionsgestörten DNA-Reparatursystemen (sog. MSIhigh-Tumoren) identifizieren, die von einer Immuntherapie mit PD-1/PD-L1-Checkpoint-Inhibitoren profitieren [234-236]. Diese machen in frühen Krankheitsstadien bis zu $1 \%$ und bei fortgeschrittenen Tumoren bis zu 2 \% aller biliären Karzinome aus [235, 237]. Zum Einsatz immunonkologischer Substanzen auch bei Patienten ohne MSI-high-Status laufen derzeit Studien.

- Genetische Veränderungen des Fibroblast Growth Factor Receptor (FGFR) werden gehäuft bei Cholangiokarzinomen beschrieben [238], insbesondere Fusionsgene mit FGFR2 wurden zwischen $13 \%$ und $17 \%$ bei intrahepatischen CCA beschrieben [239, 240]. In einer einarmigen Phase-II-Studie mit 61 Patienten mit dem spezifischen FGFR-Inhibitor Infigratinib (BGJ398) konnte bei fortgeschrittener Erkrankung ein objektives Ansprechen bei 19\% der Patienten mit FGFR2- 
Fusionen erreicht werden [241]. Weitere vielversprechende erste klinische Daten wurden z. B. auch für die Substanzen Derazantinib [242] oder Erdafitinib [243] vorgestellt. Pemigatinib wurde im April 2020 basierend auf den Ergebnissen der Phase-II-Studie FIGHT-202 für Patienten mit CCA und FGFR2Fusion zur Therapie in den USA bereits zugelassen [244]. Die Zulassung in Europa folgte im April 2021. Weitere Studien mit Medikamenten aus dieser Substanzgruppe rekrutieren derzeit Patienten in unterschiedlichen Therapielinien des CCA.

- Fusionsgene mit dem Neurotrophin-Rezeptoren TRKA, TRKB und TRKC, sog. NTRK-Genfusionen [245], sind seltene Veränderungen bei CCA, die in Einzelfällen beschrieben worden sind [246, 247]. Die große Relevanz dieser Veränderungen liegt allerdings in der hohen Ansprechrate solcher Tumoren [248], die zur tumorunabhängigen Zulassung von Larotrectinib geführt hat.

- Amplifikationen von HER2 (ERBB2) finden sich bei 5-15\% aller Gallenwegtumoren, am häufigsten bei Karzinomen der Gallenblase [249]. Erste vielversprechende Fallberichte zur Therapierelevanz dieser Veränderungen liegen vor [250-252].

- Für Patienten mit der BRAF-Mutation V600E wurde ein Ansprechen auf den BRAF-Inhibitor Vemurafenib in Einzelfällen beschrieben [253]. Inzwischen gibt es weitere Daten für ein gutes Ansprechen mit einer Kombination aus dem BRAFInhibitor Dabrafenib und dem MEK-Inhibitor Trametinib in einem frühen Bericht aus der „NCI-MATCH“-Studie [254] und weiteren Fallberichten [255-257].

- Für Patienten mit einer Mutation im Isocitrat-Dehydrogenase1(IDH1)-Gen zeigen Ergebnisse der Phase-III-ClarIDHy-Studie ein signifikant längeres medianes PFS mit 2,7 unter Ivosidenib vs. 1,4 Monaten mit Placebo, allerdings separieren sich die Kurven deutlich im längerfristigen Verlauf [258]. Das mediane Gesamtüberleben in der Studie war nicht signifikant unterschiedlich mit 10,8 Monaten mit Ivosidenib vs. 9,7 Monaten mit Placebo, allerdings wurden aus dem Placeboarm mehr als die Hälfte der Patienten nach Progress mit Ivosidenib behandelt (Cross-over-Studiendesign). Somit scheint eine Subgruppe der Patienten deutlich von der Therapie zu profitieren. Die „Disease Control Rate“, d. h. der Anteil an Patienten mit mindestens stabiler Erkrankung oder partieller Remission, lag für Ivosidenib bei $53 \%$ und für Placebo bei $28 \%$.

\subsubsection{Verlaufskontrollen unter Systemtherapie}

\begin{tabular}{|c|c|c|}
\hline 4.46) & Konsensbasierte Empfehlung & Neu 2021 \\
\hline EK & \multicolumn{2}{|c|}{$\begin{array}{l}\text { Bei biliären Karzinomen unter Systemtherapie sollte alle } \\
6-12 \text { Wochen die diagnostisch am besten geeignete } \\
\text { Schnittbildgebung durchgeführt werden. Die Interpre- } \\
\text { tation im klinischen Alltag sollte sich an den Auswerte- } \\
\text { prinzipien von RECIST } 1.1 \text { orientieren. }\end{array}$} \\
\hline & Konsens & \\
\hline
\end{tabular}

\subsection{Supportivtherapie des Hepatozellulären Karzinoms und der biliären Karzinome}

Die S3-Leitlinie Diagnostik und Therapie des Hepatozellulären Karzinoms und biliärer Karzinome umfasst 2 Tumorentitäten, und das Kapitel der Supportivtherapie wurde für beide Entitäten gemeinsam verfasst. An dieser Stelle verweisen wir daher auf die S3-Leitlinie Diagnostik und Therapie des Hepatozellulären Karzinoms.

\section{Qualitätsindikatoren}

Qualitätsindikatoren sind Messgrößen, deren Erhebung der Beurteilung der Qualität der zugrunde liegenden Strukturen, Prozesse bzw. Ergebnisse dient. Qualitätsindikatoren sind ein wichtiges Instrument des Qualitätsmanagements. Ziel ihres Einsatzes ist die stetige Verbesserung der Versorgung, indem die Ergebnisse der Versorgung dargestellt, kritisch reflektiert und wenn nötig verbessert werden. Die vorliegende Auswahl von Qualitätsindikatoren wurde gemäß der Methodik des Leitlinienprogramms Onkologie erstellt [259]. Für den Ableitungsprozess konstituierte sich eine „Arbeitsgruppe Qualitätsindikatoren“ (AG QI). Diese erstellte das finale Set der Qualitätsindikatoren auf der Grundlage der bereits bestehenden Qualitätsindikatoren der Leitlinie HCC 2013, der neuen starken Empfehlungen („soll“) der aktualisierten Leitlinie HCC/CCA, der Ergebnisse der bestehenden Qualitätsindikatoren aus den zertifizierten Leberkrebszentren (nur HCC) der Deutschen Krebsgesellschaft sowie der Ergebnisse der Recherche nach bestehenden nationalen und internationalen Qualitätsindikatoren. Die genaue Vorgehensweise und die Zusammensetzung der AG QI sind im Leitlinienreport dargelegt.

Nach 2 Onlinesitzungen dieser AG wurden 3 neue Qualitätsindikatoren (QI) angenommen, von denen 2 (QI 8 und 9) das bestehende Set ergänzen und ein QI (Typisierung CCA nach WHOKlassifikation) den bestehenden QI 1 ergänzt. Zwei QI aus dem vorbestehenden Set (QI 3 Ausbreitungsdiagnostik, QI 5 Nachsorge nach kurativem Verfahren) wurden gestrichen. 
- Tab.9 Qualitätsindikatoren.

Der Zähler ist stets eine Teilmenge des Nenners.

Die Qualitätsindikatoren 1, 4 und 6 sind mit dem onkologischen Basisdatensatz der Krebsregister zu dokumentieren (Stand: 11/2020).

Qualitätsindikator

Zugrunde liegende Empfehlung

\section{Q1 9: Inhalt Befundberichte CCA (neu 2020)}

Z: Patienten des Nenners, bei denen ein histopathologischer Befundbericht mit folgenden Angaben vorliegt:

- Staging (TNM-Klassifikation)

- Typing (WHO-Klassifikation)

- Grading

- Resektionsrand (R-Klassifikation)

- Bei intrahepatischen Cholangiokarzinomen (C22.1): Status der nicht tumorösen Leber $\mathrm{N}$ : Alle Patienten mit CCA und Resektion oder Explantation
4.0

Die Bearbeitung und Befundung eines Resektats soll die Ausdehnung des Tumors (Staging) gemäß der aktuellen TNM-Klassifikation, seinen Typ (Typing) und Differenzierungsgrad (Grading) und den Status des Resektatrandes (R-Klassifikation) sowie bei intrahepatischen Cholangiokarzinomen den Status der nicht tumorösen Leber ermitteln. Bei Präparaten mit prämalignen Läsionen soll durch genaue Aufarbeitung ein möglicher Übergang in ein invasives Karzinom abgeklärt werden.
Qualitätsziel: Möglichst häufig vollständige Befundberichte

\section{Anhang}

\subsection{Literaturübersichten}

\subsubsection{Kapitel 4.4. Operative und Interventionelle Therapie der biliären Karzinome}

- Tab.10 Übersicht über Literatur zur präoperativen biliären Drainage (PBD).

\begin{tabular}{|c|c|c|c|}
\hline Autor & Design & Methode & Ergebnis \\
\hline Coelen 2018 [163] & $\begin{array}{l}\text { Randomisiert, } \\
\text { multizentrisch }\end{array}$ & $\begin{array}{l}\text { ERCP vs. PTCD für die voraussichtlich } \\
\text { verbleibende Leberseite bei geplan- } \\
\text { ter Hemihepatektomie }\end{array}$ & $\begin{array}{l}\text { Höhere Mortalität in der PTBD-Gruppe ( } 41 \% \text { von } \\
27 \text { Patienten) als in endoskopischer BD-Gruppe ( } 11 \% \text { von } \\
27 \text { Patienten) mit einem relativen Risiko von } 3,7(\mathrm{p}=0,03) \text {. }\end{array}$ \\
\hline Celotti 2017 [260] & Metaanalyse & PBD vs. keine PBD & Gleiche Mortalität, erhöhte Morbidität bei PBD \\
\hline Ba 2020 [164] & Retrospektiv & PTCD vs ERCP & $\begin{array}{l}\text { ERCP-Gruppe hatte im Vergleich zur PTBD-Gruppe eine } \\
\text { höhere Inzidenz einer postprozeduralen Cholangitis } \\
(37[37,37 \%] \text { vs. } 18[22,22 \%], P=0,028) \text { und Pankreatitis } \\
(17[17,17 \%] \text { vs. } 2[2,47 \%], P=0,001) \text {; diese Gruppe benö- } \\
\text { tigte häufiger eine salvaged biliary drainage ( } 18 \text { [ } 18,18 \%] \\
\text { vs. } 5[6,17 \%], P=0,029) \text {, und erzeugte höhere Kosten } \\
(P<0,05)\end{array}$ \\
\hline Ramanathan 2018 [261] & Retrospektiv & ERCP & Mehr postoperative Komplikationen bei PBD \\
\hline Cai 2017 [262] & $\begin{array}{l}\text { Retrospektiv, } \\
\text { Single-Center }\end{array}$ & & PBD empfohlen, falls Bilirubin $>12,4 \mathrm{mg} / \mathrm{dL}$ \\
\hline Farges 2013 [263] & $\begin{array}{l}\text { Retrospektiv, } \\
\text { Multi-Center }\end{array}$ & ERCP & $\begin{array}{l}\text { Erniedrigte postoperative Mortalität im Falle einer PBD, falls } \\
\text { Hemihepatektomie rechts erfolgt ist }\end{array}$ \\
\hline Xiong 2013 [264] & $\begin{array}{l}\text { Retrospektiv, } \\
\text { Single-Center }\end{array}$ & & \\
\hline Wang 2019 [265] & Retrospektiv & ,seeding metastasis‘: ERCP vs. PTCD & $\begin{array}{l}\text { ERCP besser als PTCD in Bezug auf Metastasenaussaat bei } \\
(10,5 \% \text { vs. } 22,0 \%, O R=0,35,95 \%-K I 0,23 ; 0,53)\end{array}$ \\
\hline Wronka 2019 [160] & $\begin{array}{l}\text { Retrospektiv, } \\
\text { Single-Center }\end{array}$ & PBD vs. keine PBD & $\begin{array}{l}\text { Erhöhte postop. Mortalität bei Bili >6,2; erhöhte Morbidität } \\
\text { bei Bili > 2,5 mg/dl }\end{array}$ \\
\hline Kishi 2016 [266] & Retrospektiv & & \\
\hline Nakai 2018 [166] & Retrospektiv & $\begin{array}{l}\text { Nasobiliäre Sonde vs. Plastikstent } \\
\text { transpapillär }\end{array}$ & Vergleichbar \\
\hline
\end{tabular}


- Tab. 10 (Fortsetzung)

\begin{tabular}{|c|c|c|c|}
\hline Autor & Design & Methode & Ergebnis \\
\hline Komaya 2017 [167] & Retrospektiv & PTCD vs. endoskopische Drainage & $\begin{array}{l}\text { Das OS ist bei der PTBD signifikant niedriger als in der } \\
\text { endoskopischen Drainage-Gruppe ( } 37,0 \% \text { vs. } 44,3 \% \text { at } \\
5 \text { years, } p=0,019 \text { ). } \\
\text { Die PTBD stellte einen Risikofaktor für Stichkanalmetasta- } \\
\text { sen }(p=0,005) \text { dar. }\end{array}$ \\
\hline Kim 2015 [168] & Retrospektiv & PTCD vs. endoskopische Drainage & $\begin{array}{l}\text { Höhere Morbidität nach PTCD; in } 2 \text { von } 62 \text { Fällen Tumor- } \\
\text { zellverschleppung (seeding metastasis) nach PTCD. }\end{array}$ \\
\hline
\end{tabular}

- Tab. 11 Übersicht über Literatur zur biliären Drainage (BD) bei Cholangiokarzinom.

\begin{tabular}{|c|c|c|c|c|c|c|}
\hline Autor & Design & Methode & \multicolumn{4}{|l|}{ Ergebnis } \\
\hline Abraham 2002 [172] & Retrospektiv & & \multicolumn{4}{|c|}{$\begin{array}{l}\text {,Quality of Life' bei Hyperbilirubinämie schlechter und } \\
\text { durch erfolgreiche Drainage zu verbessern }\end{array}$} \\
\hline Paik 2009 [173] & Retrospektiv & PTCD vs. ERCP & \multicolumn{4}{|c|}{$\begin{array}{l}\text { Überleben nach erfolgreicher Drainage besser als keine } \\
\text { Drainage }\end{array}$} \\
\hline Smith 1994 [178] & RCT & $\begin{array}{l}\text { Distaler maligner Gallenwegver- } \\
\text { schluss: Chirurgie/bilio-dig. Anasto- } \\
\text { mose vs. Endoskopische Drainage }\end{array}$ & \multicolumn{4}{|c|}{ Endoskopie mit weniger (frühen) Komplikationen } \\
\hline \multirow[t]{3}{*}{ Speer 1987 [179] } & \multirow[t]{3}{*}{ RCT } & \multirow[t]{3}{*}{ Palliative Drainage, PTCD vs. ERCP } & & \multicolumn{3}{|c|}{ PLASTIK UNI-lateral } \\
\hline & & & Ikterus behoben & $81 \%$ & $61 \%$ & $p=0,017$ \\
\hline & & & 30-d Mortalität & $15 \%$ & $33 \%$ & $p=0,016$ \\
\hline De Palma 2001 [189] & RCT & Uni- vs. bilateral & \multicolumn{4}{|c|}{$\begin{array}{l}\text { - Unilateral kann genügen! } \\
\text { - Hinweis auf Cholangitis durch Intervention }\end{array}$} \\
\hline Saluja 2008 [174] & RCT & $\begin{array}{l}\text { PTCD vs. ERCP bei hilär einwachsen- } \\
\text { dem Gallenblasenkarzinom }\end{array}$ & \multicolumn{4}{|c|}{$\begin{array}{l}\text { " PTCD erfolgreicher, } \\
\text { - PTCD mit weniger Cholangitis }\end{array}$} \\
\hline Sangchan 2012 [267] & & SEMS vs. PLASTIK & \multicolumn{4}{|c|}{$\begin{array}{l}\text { - SEMS bessere primäre Drainage } \\
\text { - Aber: Plastik: } 66 \% 7 \mathrm{Fr}\end{array}$} \\
\hline Cheng 2002 [192] & Retrospektiv & SEMS vs. Plastik vs. PTCD & \multicolumn{4}{|c|}{ SEMS, Bihilär, $69 \%$ ohne erneute Intervention } \\
\hline Lee 2019 [181] & RANDOMISIERTE STUDIE & $\begin{array}{l}\text { Malignant hilar stricture: side-byside } \\
\text { vs. stent in stent SEMS }\end{array}$ & \multicolumn{4}{|l|}{ Similar Efficacy } \\
\hline Uberoi 2012 [177] & Retrospektiv & Registerstudie Großbritannien & \multicolumn{4}{|c|}{ Krankenhausmortalität 19,8\% bei maligner Stenose } \\
\hline Paik 2018 [183] & $\mathrm{RCT}$ & $\begin{array}{l}\text { EUS vs. ERCP bei distalem biliären } \\
\text { Verschluss }\end{array}$ & \multicolumn{4}{|l|}{ Ebenbürtig } \\
\hline Bang 2018 [184] & $\mathrm{RCT}$ & $\begin{array}{l}\text { EUS vs. ERCP bei distalem biliären } \\
\text { Verschluss }\end{array}$ & \multicolumn{4}{|l|}{ Ebenbürtig } \\
\hline
\end{tabular}


- Tab. 12 Übersicht über Literatur zu intraduktalen, lokal ablativen Verfahren (PBD).

\begin{tabular}{|c|c|c|c|}
\hline Autor & Design & Methode & Ergebnis \\
\hline Ortner 2003 [199] & Randomisiert & PDT+ Stent vs Stent & Überlebensvorteil PDT \\
\hline Zoepf 2001 [197] & Kohortenstudie & PDT mit 5-ALA & Nicht effektiv \\
\hline Zoepf 2005 [200] & Randomisiert & PDT+ Stent vs. Stent & Überlebensvorteil PDT \\
\hline Pereira 2018 [201] & Randomisiert & PDT+ Stent vs. Stent & $\begin{array}{l}\text { Schlechteres Outcome bei Patienten mit lokal } \\
\text { fortgeschrittenen und metastasierten perihilären } \\
\text { Tumoren }\end{array}$ \\
\hline Kahaleh 2008 [206] & Retrospektiv & PDT+ Stent vs. Stent & Überlebensvorteil PDT \\
\hline $\begin{array}{l}\text { Gonzalez-Carmona } \\
2019 \text { [202] }\end{array}$ & Retrospektiv & PDT + Ctx + Stent vs. Ctx + Stent & Überlebensvorteil PDT \\
\hline Strand 2014 [204] & Retrospektiv & PDT vs. RFA & $\begin{array}{l}\text { Überleben nach ERCP-geführter RFA und PDT ergab } \\
\text { keinen signifikanten Unterschied bei Patienten mit } \\
\text { einem irresektablen CCA. }\end{array}$ \\
\hline Yang 2018 [198] & Randomisiert & $\begin{array}{l}\text { RFA + stent vs. stent/ } \\
\text { Bismuth } 1+2 \text { perihiläres CCA }\end{array}$ & Überlebensvorteil RFA \\
\hline Wentrup 2016 [203] & Retrospektiv & $\mathrm{PDT}+\mathrm{CT}$ x vs. PDT alone & $\begin{array}{l}\text { 1-Jahres-Überlebensrate war signifikant höher in der } \\
\text { PDT-CT-Gruppe verglichen mit der PDT-alone- } \\
\text { Gruppe ( } 88 \% \text { vs. } 58 \%, p=0,001) \text {. }\end{array}$ \\
\hline Dolak 2017 [205] & Retrospektiv & PDT & $\begin{array}{l}\text { PDT mit Polyhematoporphyrin war durchführbar und } \\
\text { sicher. }\end{array}$ \\
\hline Tal 2014 [195] & Retrospektiv & Endoskopische RFA & $\begin{array}{l}\text { Endoskopische RFA ist einfach und sicher. Hyperbili- } \\
\text { rubinämie war eine Nebenwirkung bei } 3 \text { Patienten. } \\
\text { Größere Studienpopulationen sind notwendig zur } \\
\text { weiteren Untersuchung der Sicherheit und Effizienz. }\end{array}$ \\
\hline
\end{tabular}

\section{Abbildungsverzeichnis}

- Abb. 1 Diagnosealgorithmus eines Patienten mit einem Verdacht auf ein Cholangiokarzinom
- Tab. 11 Übersicht über Literatur zur biliären Drainage (BD) bei Cholangiokarzinom

- Tab. 12 Übersicht über Literatur zu intraduktalen, lokal ablativen Verfahren (PBD)
219

220

\section{Tabellenverzeichnis}

\begin{tabular}{|l|c|}
\hline $\begin{array}{l}\text { Tab. } 1 \text { Beteiligte Fachgesellschaften und Organisa- } \\
\text { tionen }\end{array}$ & 192 \\
\hline \begin{tabular}{l} 
Tab. 2 Arbeitsgruppen und deren Mitglieder \\
\hline Tab. 3 Beteiligte Experten
\end{tabular} & 193 \\
\hline $\begin{array}{l}\text { Tab. } 4 \text { Schema der Evidenzgraduierung nach Oxford } \\
\text { (Version 2011) [1] }\end{array}$ & 193 \\
\hline \begin{tabular}{l} 
Tab. 5 Schema der Empfehlungsgraduierung \\
\hline Tab. 6 Konsensstärke
\end{tabular} & 196 \\
\hline $\begin{array}{l}\text { Tab. } 7 \text { Typische Merkmale der CCA-Subtypen } \\
\text { [42-45] }\end{array}$ & 196 \\
\hline $\begin{array}{l}\text { Tab. } 8 \text { Molekulare Alterationen beim small duct } \\
\text { iCCA }\end{array}$ & 201 \\
\hline $\begin{array}{l}\text { Tab. } 9 \text { Qualitätsindikatoren } \\
\text { T Tab. } 10 \text { Übersicht über Literatur zur präoperativen } \\
\text { biliären Drainage (PBD) }\end{array}$ & 203 \\
\hline
\end{tabular}

Interessenkonflikt

Die Übersicht über die Interessenkonflikte der Autorinnen und Autoren sind im Leitlinienreport veröffentlicht.

\section{Literatur}

[1] Howick ] et al. The 2011 Oxford CEBM Evidence Levels of Evidence (Introductory Document). Available from: 2011 http://www.cebm.net/index. aspx?o $=5653$

[2] Arbeitsgemeinschaft der Wissenschaftlichen Medizinischen Fachgesellschaften - Ständige Kommission L. AWMF-Regelwerk „Leitlinien“. 2012. [cited 09.12.2013; Available from: 1. Auflage: http://www.awmf.org/ leitlinien/awmf-regelwerk/awmf-regelwerk.html

[3] Atchison EA et al. Risk of cancer in a large cohort of U.S. veterans with diabetes. Int J Cancer 2011; 128 (3): 635-643

[4] de Valle MB, Björnsson E, Lindkvist B. Mortality and cancer risk related to primary sclerosing cholangitis in a Swedish population-based cohort. Liver Int 2012; 32 (3): 441-448 
[5] El-Serag HB et al. Risk of hepatobiliary and pancreatic cancers after hepatitis $C$ virus infection: A population-based study of U.S. veterans. Hepatology 2009; 49 (1): 116-123

[6] Huang $Y$ et al. Smoking and risk of cholangiocarcinoma: a systematic review and meta-analysis. Oncotarget 2017; 8 (59): 100570-100581

[7] Jing $W$ et al. Diabetes mellitus and increased risk of cholangiocarcinoma: a meta-analysis. Eur J Cancer Prev 2012; 21 (1): 24-31

[8] Palmer WC, Patel T. Are common factors involved in the pathogenesis of primary liver cancers? A meta-analysis of risk factors for intrahepatic cholangiocarcinoma. J Hepatol 2012; 57 (1): 69-76

[9] Wongjarupong $\mathrm{N}$ et al. Non-alcoholic fatty liver disease as a risk factor for cholangiocarcinoma: a systematic review and meta-analysis. BMC Gastroenterol 2017; 17 (1): 149

[10] Park JY et al. Long-term follow up of gallbladder polyps. J Gastroenterol Hepatol 2009; 24 (2): 219-222

[11] Nagaraja V, Eslick GD. Systematic review with meta-analysis: the relationship between chronic Salmonella typhi carrier status and gall-bladder cancer. Aliment Pharmacol Ther 2014; 39 (8): 745-750

[12] Razumilava N, Gores G]. Cholangiocarcinoma. Lancet 2014; 383: 2168 2179

[13] Rizvi S et al. Cholangiocarcinoma - evolving concepts and therapeutic strategies. Nat Rev Clin Oncol 2018; 15 (2): 95-111

[14] Valle JW et al. Biliary cancer: ESMO Clinical Practice Guidelines for diagnosis, treatment and follow-up. Ann Oncol 2016; 27 (Suppl. 5): v28-v37

[15] Rizvi S, Gores G]. Pathogenesis, diagnosis, and management of cholangiocarcinoma. Gastroenterology 2013; 145 (6): 1215-1229

[16] Kamsa-ard S et al. Risk Factors for Cholangiocarcinoma in Thailand: A Systematic Review and Meta-Analysis. Asian Pac J Cancer Prev 2018; 19 (3): 605-614

[17] Qian MB et al. Clonorchiasis. Lancet 2016; 387: 800-810

[18] Qian MB, Zhou XN. Global burden of cancers attributable to liver flukes. Lancet Glob Health 2017; 5 (2): e139

[19] You MS et al. Natural Course and Risk of Cholangiocarcinoma in Patients with Recurrent Pyogenic Cholangitis: A Retrospective Cohort Study. Gut Liver 2019; 13 (3): 373-379

[20] Ten Hove A et al. Meta-analysis of risk of developing malignancy in congenital choledochal malformation. Br J Surg 2018; 105 (5): 482-490

[21] Fahrner R, Dennler SG, Inderbitzin D. Risk of malignancy in Caroli disease and syndrome: A systematic review. World J Gastroenterol 2020; 26 (31): 4718-4728

[22] Claessen MM et al. High lifetime risk of cancer in primary sclerosing cholangitis. J Hepatol 2009; 50 (1): 158-164

[23] Tyson GL, El-Serag HB. Risk factors for cholangiocarcinoma. Hepatology 2011; 54 (1): 173-184

[24] McGee EE et al. Smoking, Alcohol, and Biliary Tract Cancer Risk: A Pooling Project of 26 Prospective Studies. J Natl Cancer Inst 2019; 111 (12): 1263-1278

[25] Petrick JL et al. Body Mass Index, Diabetes and Intrahepatic Cholangiocarcinoma Risk: The Liver Cancer Pooling Project and Meta-analysis. Am J Gastroenterol 2018; 113 (10): 1494-1505

[26] Clements $\mathrm{O}$ et al. Risk factors for intrahepatic and extrahepatic cholangiocarcinoma: A systematic review and meta-analysis. J Hepatol 2020; 72 (1): 95-103

[27] Schmidt MA, Marcano-Bonilla L, Roberts LR. Gallbladder cancer: epidemiology and genetic risk associations. Chin Clin Oncol 2019; 8 (4): 31

[28] Rawla P et al. Epidemiology of gallbladder cancer. Clin Exp Hepatol 2019; 5 (2): 93-102

[29] Kratzer W et al. [Gallbladder polyps: prevalence and risk factors]. Ultraschall Med 2011; 32 (Suppl. 1): S68-S73

[30] Schnelldorfer T. Porcelain gallbladder: a benign process or concern for malignancy? J Gastrointest Surg 2013; 17 (6): 1161-1168
[31] Des/ardins H et al. Porcelain Gallbladder: Is Observation a Safe Option in Select Populations? J Am Coll Surg 2018; 226 (6): 1064-1069

[32] Patel $S$ et al. Hyalinizing cholecystitis and associated carcinomas: clinicopathologic analysis of a distinctive variant of cholecystitis with porcelain-like features and accompanying diagnostically challenging carcinomas. Am J Surg Pathol 2011; 35 (8): 1104-1113

[33] Gutt C et al. [Updated S3-Guideline for Prophylaxis, Diagnosis and Treatment of Gallstones. German Society for Digestive and Metabolic Diseases (DGVS) and German Society for Surgery of the Alimentary Tract (DGAV) - AWMF Registry 021/008]. Z Gastroenterol 2018; 56 (8): 912966

[34] Eaton JE, Thackeray EW, Lindor KD. Likelihood of malignancy in gallbladder polyps and outcomes following cholecystectomy in primary sclerosing cholangitis. Am J Gastroenterol 2012; 107 (3): 431-439

[35] [Practice guideline autoimmune liver diseases - AWMF-Reg. No. 02127]. Z Gastroenterol 2017; 55 (11): 1135-1226

[36] Wiles R et al. Management and follow-up of gallbladder polyps: Joint guidelines between the European Society of Gastrointestinal and Abdominal Radiology (ESGAR), European Association for Endoscopic Surgery and other Interventional Techniques (EAES), International Society of Digestive Surgery - European Federation (EFISDS) and European Society of Gastrointestinal Endoscopy (ESGE). Eur Radiol 2017; 27 (9): 3856-3866

[37] Fung BM, Lindor KD, Tabibian JH. Cancer risk in primary sclerosing cholangitis: Epidemiology, prevention, and surveillance strategies. World J Gastroenterol 2019; 25 (6): 659-671

[38] Charatcharoenwitthaya P et al. Utility of serum tumor markers, imaging, and biliary cytology for detecting cholangiocarcinoma in primary sclerosing cholangitis. Hepatology 2008; 48 (4): 1106-1117

[39] Naitoh I et al. Predictive factors for positive diagnosis of malignant biliary strictures by transpapillary brush cytology and forceps biopsy. J Dig Dis 2016; 17 (1): 44-51

[40] Navaneethan U et al. Comparative effectiveness of biliary brush cytology and intraductal biopsy for detection of malignant biliary strictures: a systematic review and meta-analysis. Gastrointest Endosc 2015; 81 (1): $168-176$

[41] Klimstra DS, Paradis V, Schirmacher P. Tumors of the gallbladder and extrahepatic bile ducts. In: WHO Classification of Tumours Editorial Board WHO-Classification of Tumours (5th ed.) Digestive System Tumours. Lyon: International Agency for Research on Cancer; 2019: 265294

[42] Paradis V, Park FM, Schirmacher P. Tumors of the liver and intrahepatic bile ducts. In: WHO Classification of Tumours Editorial Board WHOClassification of Tumours (5th ed.) Digestive System Tumours. Lyon: International Agency for Research on Cancer; 2019: 215-264

[43] Liau JY et al. Morphological subclassification of intrahepatic cholangiocarcinoma: etiological, clinicopathological, and molecular features. Mod Pathol 2014; 27 (8): 1163-1173

[44] Nakamura $\mathrm{H}$ et al. Genomic spectra of biliary tract cancer. Nat Genet 2015; 47 (9): 1003-1010

[45] Sasaki A et al. Intrahepatic peripheral cholangiocarcinoma: mode of spread and choice of surgical treatment. Br J Surg 1998; 85 (9): 12061209

[46] Moeini A et al. Mixed hepatocellular cholangiocarcinoma tumors: Cholangiolocellular carcinoma is a distinct molecular entity. J Hepatol 2017; 66 (5): 952-961

[47] Paradis V, Singh SP. Other tumours of the digestive system. In: WHO Classification of Tumours Editorial Board WHO-Classification of Tumours (5th ed.) Digestive System Tumours. Lyon: International Agency for Research on Cancer; 2019: 499-510

[48] Wittekind C. TNM-Klassifikation maligner Tumoren. (8. Auflage, korrigierter Nachdruck). Weinheim: Wiley-VCH; 2020 
[49] Wagner GHP. Organspezifische Tumordokumentation - Prinzipien und Verschlüsselungsanweisungen für Klinik und Praxis. Online-version: deutsche Krebsgesellschaft. Frankfurt (Main), 1995

[50] Khuntikeo $\mathrm{N}$ et al. Cohort profile: cholangiocarcinoma screening and care program (CASCAP). BMC Cancer 2015; 15: 459

[51] Li R et al. Dynamic enhancing vascular pattern of intrahepatic peripheral cholangiocarcinoma on contrast-enhanced ultrasound: the influence of chronic hepatitis and cirrhosis. Abdom Imaging 2013; 38 (1): 112-119

[52] Xu HX et al. Contrast-enhanced ultrasound of intrahepatic cholangiocarcinoma: correlation with pathological examination. $\mathrm{Br}$ J Radiol 2012; 85: 1029-1037

[53] Wildner D et al. CEUS in hepatocellular carcinoma and intrahepatic cholangiocellular carcinoma in 320 patients - early or late washout matters: a subanalysis of the DEGUM multicenter trial. Ultraschall Med 2015; 36 (2): 132-139

[54] Bach AM et al. Portal vein evaluation with US: comparison to angiography combined with CT arterial portography. Radiology 1996; 201 (1): 149-154

[55] Wennmacker SZ et al. Transabdominal ultrasound and endoscopic ultrasound for diagnosis of gallbladder polyps. Cochrane Database Syst Rev 2018; 8: CD012233

[56] Zhang Y et al. Intrahepatic peripheral cholangiocarcinoma: comparison of dynamic CT and dynamic MRI. J Comput Assist Tomogr 1999; 23 (5): 670-677

[57] Johnson PT, Fishman EK. Routine use of precontrast and delayed acquisitions in abdominal CT: time for change. Abdom Imaging 2013; 38 (2): 215-223

[58] Fabrega-Foster $\mathrm{K}$ et al. Multimodality imaging of intrahepatic cholangiocarcinoma. Hepatobiliary Surg Nutr 2017; 6 (2): 67-78

[59] Valls C et al. Intrahepatic peripheral cholangiocarcinoma: CT evaluation. Abdom Imaging 2000; 25 (5): 490-496

[60] $\mathrm{Kim} \mathrm{JH}$ et al. Radiofrequency ablation for the treatment of primary intrahepatic cholangiocarcinoma. Am J Roentgenol 2011; 196 (2): W205W209

[61] Bridgewater ] et al. Guidelines for the diagnosis and management of intrahepatic cholangiocarcinoma. J Hepatol 2014; 60 (6): 1268-1289

[62] Jhaveri KS, Hosseini-Nik H. MRI of cholangiocarcinoma. J Magn Reson Imaging 2015; 42 (5): 1165-1179

[63] Murakami T et al. Contrast-enhanced MR imaging of intrahepatic cholangiocarcinoma: pathologic correlation study. J Magn Reson Imaging 1995; 5 (2): 165-170

[64] Hamrick-Turner J, Abbitt PL, Ros PR. Intrahepatic cholangiocarcinoma: MR appearance. Am J Roentgenol 1992; 158 (1): 77-79

[65] Fan ZM et al. Intrahepatic cholangiocarcinoma: spin-echo and contrastenhanced dynamic MR imaging. Am J Roentgenol 1993; 161 (2): $313-$ 317

[66] Sheng RF et al. MRI of small intrahepatic mass-forming cholangiocarcinoma and atypical small hepatocellular carcinoma $(<1=3 \mathrm{~cm})$ with cirrhosis and chronic viral hepatitis: a comparative study. Clin Imaging 2014; 38 (3): 265-272

[67] Chung YE et al. Varying appearances of cholangiocarcinoma: radiologicpathologic correlation. Radiographics 2009; 29 (3): 683-700

[68] Park HJ et al. Small intrahepatic mass-forming cholangiocarcinoma: target sign on diffusion-weighted imaging for differentiation from hepatocellular carcinoma. Abdom Imaging 2013; 38 (4): 793-801

[69] Fattach HE et al. Intrahepatic and hilar mass-forming cholangiocarcinoma: Qualitative and quantitative evaluation with diffusion-weighted MR imaging. Eur J Radiol 2015; 84 (8): 1444-1451

[70] Navaneethan U et al. Endoscopic ultrasound in the diagnosis of cholangiocarcinoma as the etiology of biliary strictures: a systematic review and meta-analysis. Gastroenterol Rep (Oxf) 2015; 3 (3): 209-215
[71] Pahade JK et al. Is there an added value of a hepatobiliary phase with gadoxetate disodium following conventional MRI with an extracellular gadolinium agent in a single imaging session for detection of primary hepatic malignancies? Abdom Radiol (NY) 2016; 41 (7): 1270-1284

[72] Park H] et al. The role of diffusion-weighted MR imaging for differentiating benign from malignant bile duct strictures. Eur Radiol 2014; 24 (4): 947-958

[73] Lee J et al. Mass-forming Intrahepatic Cholangiocarcinoma: Diffusionweighted Imaging as a Preoperative Prognostic Marker. Radiology 2016; 281 (1): 119-128

[74] Rupp C et al. Effect of scheduled endoscopic dilatation of dominant strictures on outcome in patients with primary sclerosing cholangitis. Gut 2019; 68 (12): 2170-2178

[75] Zhang $\mathrm{H}$ et al. Radiological Imaging for Assessing the Respectability of Hilar Cholangiocarcinoma: A Systematic Review and Meta-Analysis. Biomed Res Int 2015; 2015: 497942

[76] Lamarca A et al. (18)F-fluorodeoxyglucose positron emission tomography ((18)FDG-PET) for patients with biliary tract cancer: Systematic review and meta-analysis. J Hepatol 2019; 71 (1): 115-129

[77] Feng ST et al. Cholangiocarcinoma: spectrum of appearances on GdEOB-DTPA-enhanced MR imaging and the effect of biliary function on signal intensity. BMC Cancer 2015; 15: 38

[78] Kim SH et al. Typical and atypical imaging findings of intrahepatic cholangiocarcinoma using gadolinium ethoxybenzyl diethylenetriamine pentaacetic acid-enhanced magnetic resonance imaging. J Comput Assist Tomogr 2012; 36 (6): 704-709

[79] De Moura DTH et al. Endoscopic retrograde cholangiopancreatography versus endoscopic ultrasound for tissue diagnosis of malignant biliary stricture: Systematic review and meta-analysis. Endosc Ultrasound 2018; 7 (1): 10-19

[80] Heimbach JK et al. Trans-peritoneal fine needle aspiration biopsy of hilar cholangiocarcinoma is associated with disease dissemination. HPB (Oxford) 2011; 13 (5): 356-360

[81] El Chafic AH et al. Impact of preoperative endoscopic ultrasound-guided fine needle aspiration on postoperative recurrence and survival in cholangiocarcinoma patients. Endoscopy 2013; 45 (11): 883-889

[82] Korc P, Sherman S. ERCP tissue sampling. Gastrointest Endosc 2016; 84 (4): 557-571

[83] Fogel EL et al. Effectiveness of a new long cytology brush in the evaluation of malignant biliary obstruction: a prospective study. Gastrointest Endosc 2006; 63 (1): 71-77

[84] Shieh FK et al. Improved endoscopic retrograde cholangiopancreatography brush increases diagnostic yield of malignant biliary strictures. World J Gastrointest Endosc 2014; 6 (7): 312-317

[85] Glasbrenner B et al. Prospective evaluation of brush cytology of biliary strictures during endoscopic retrograde cholangiopancreatography. Endoscopy 1999; 31 (9): 712-717

[86] Macken E et al. Brush cytology of ductal strictures during ERCP. Acta Gastroenterol Belg 2000; 63 (3): 254-259

[87] Mansfield JC et al. A prospective evaluation of cytology from biliary strictures. Gut 1997; 40 (5): 671-677

[88] Trikudanathan $G$ et al. Diagnostic yield of bile duct brushings for cholangiocarcinoma in primary sclerosing cholangitis: a systematic review and meta-analysis. Gastrointest Endosc 2014; 79 (5): 783-789

[89] Draganov PV et al. Diagnostic accuracy of conventional and cholangioscopy-guided sampling of indeterminate biliary lesions at the time of ERCP: a prospective, long-term follow-up study. Gastrointest Endosc 2012; 75 (2): 347-353

[90] Sugiyama M et al. Endoscopic transpapillary bile duct biopsy without sphincterotomy for diagnosing biliary strictures: a prospective comparative study with bile and brush cytology. Am J Gastroenterol 1996; 91 (3): 465-467 
[91] Jailwala J et al. Triple-tissue sampling at ERCP in malignant biliary obstruction. Gastrointest Endosc 2000; 51 (4): 383-390

[92] Hartman DJ et al. Tissue yield and diagnostic efficacy of fluoroscopic and cholangioscopic techniques to assess indeterminate biliary strictures. Clin Gastroenterol Hepatol 2012; 10 (9): 1042-1046

[93] Pugliese V et al. Endoscopic retrograde forceps biopsy and brush cytology of biliary strictures: a prospective study. Gastrointest Endosc 1995; 42 (6): 520-526

[94] Kitajima Y et al. Usefulness of transpapillary bile duct brushing cytology and forceps biopsy for improved diagnosis in patients with biliary strictures. J Gastroenterol Hepatol 2007; 22 (10): 1615-1620

[95] Navaneethan $U$ et al. Single-operator cholangioscopy and targeted biopsies in the diagnosis of indeterminate biliary strictures: a systematic review. Gastrointest Endosc 2015; 82 (4): 608-614 e2

[96] Gerges C et al. Digital single-operator peroral cholangioscopy-guided biopsy versus ERCP-guided brushing for indeterminate biliary strictures: a prospective, randomized multicenter trial (with video). Gastrointest Endosc 2019; 91: 1105-1113

[97] Aabakken L et al. Role of endoscopy in primary sclerosing cholangitis: European Society of Gastrointestinal Endoscopy (ESGE) and European Association for the Study of the Liver (EASL) Clinical Guideline. Endoscopy 2017; 49 (6): 588-608

[98] Bagante F et al. Assessment of the Lymph Node Status in Patients Undergoing Liver Resection for Intrahepatic Cholangiocarcinoma: the New Eighth Edition AJCC Staging System. J Gastrointest Surg 2018; 22 (1): 52-59

[99] Bagante F et al. Surgical Management of Intrahepatic Cholangiocarcinoma in Patients with Cirrhosis: Impact of Lymphadenectomy on PeriOperative Outcomes. World J Surg 2018; 42 (8): 2551-2560

[100] Ebata T et al. Surgical resection for Bismuth type IV perihilar cholangiocarcinoma. Br J Surg 2018; 105 (7): 829-838

[101] El-Diwany R, Pawlik TM, Ejaz A. Intrahepatic Cholangiocarcinoma. Surg Oncol Clin N Am 2019; 28 (4): 587-599

[102] Lang $\mathrm{H}$ et al. Operations for intrahepatic cholangiocarcinoma: singleinstitution experience of 158 patients. J Am Coll Surg 2009; 208 (2): $218-228$

[103] Schnitzbauer AA et al. The MEGNA Score and Preoperative Anemia are Major Prognostic Factors After Resection in the German Intrahepatic Cholangiocarcinoma Cohort. Ann Surg Oncol 2020; 27 (4): 1147-1155

[104] Zhang XF et al. Perioperative and Long-Term Outcome for Intrahepatic Cholangiocarcinoma: Impact of Major Versus Minor Hepatectomy. J Gastrointest Surg 2017; 21 (11): 1841-1850

[105] Bartsch F et al. Extended resection of intrahepatic cholangiocarcinoma: A retrospective single-center cohort study. Int J Surg 2019; 67: 62-69

[106] Mizuno T, Ebata T, Nagino M. Advanced hilar cholangiocarcinoma: An aggressive surgical approach for the treatment of advanced hilar cholangiocarcinoma: Perioperative management, extended procedures, and multidisciplinary approaches. Surg Oncol 2020; 33: 201-206

[107] Rassam F et al. Modern work-up and extended resection in perihilar cholangiocarcinoma: the AMC experience. Langenbecks Arch Surg 2018; 403 (3): 289-307

[108] Primrose JN et al. Capecitabine compared with observation in resected biliary tract cancer (BILCAP): a randomised, controlled, multicentre, phase 3 study. Lancet Oncol 2019; 20 (5): 663-673

[109] Le Roy B et al. Neoadjuvant chemotherapy for initially unresectable intrahepatic cholangiocarcinoma. Br J Surg 2018; 105 (7): 839-847

[110] Chang Y et al. Impact of surgical strategies on the survival of gallbladder cancer patients: analysis of 715 cases. World J Surg Oncol 2020; 18 (1): 142

[111] Coimbra FJF et al. BRAZILIAN CONSENSUS ON INCIDENTAL GALLBLADDER CARCINOMA. Arq Bras Cir Dig 2020; 33 (1): e1496
[112] Sikora SS, Singh RK. Surgical strategies in patients with gallbladder cancer: nihilism to optimism. J Surg Oncol 2006; 93 (8): 670-681

[113] Søreide K et al. Systematic review of management of incidental gallbladder cancer after cholecystectomy. Br J Surg 2019; 106 (1): 32-45

[114] Benson AB 3rd et al. NCCN clinical practice guidelines in oncology: hepatobiliary cancers. J Natl Compr Canc Netw 2009; 7 (4): 350-391

[115] Yuza K et al. Long-term outcomes of surgical resection for T1b gallbladder cancer: an institutional evaluation. BMC Cancer 2020; 20 (1): 20

[116] Lee SE et al. Surgical strategy for T1 gallbladder cancer: a nationwide multicenter survey in South Korea. Ann Surg Oncol 2014; 21 (11): 3654-3660

[117] Bartsch F et al. Surgical Resection for Recurrent Intrahepatic Cholangiocarcinoma. World J Surg 2019; 43 (4): 1105-1116

[118] Spolverato G et al. Management and Outcomes of Patients with Recurrent Intrahepatic Cholangiocarcinoma Following Previous Curative-Intent Surgical Resection. Ann Surg Oncol 2016; 23 (1): 235-243

[119] Seidensticker R et al. Extensive Use of Interventional Therapies Improves Survival in Unresectable or Recurrent Intrahepatic Cholangiocarcinoma. Gastroenterol Res Pract 2016; 2016: 8732521

[120] Xu C et al. Ultrasound-guided percutaneous microwave ablation versus surgical resection for recurrent intrahepatic cholangiocarcinoma: intermediate-term results. Int J Hyperthermia 2019; 36 (1): 351-358

[121] Zhang SJ et al. Thermal ablation versus repeated hepatic resection for recurrent intrahepatic cholangiocarcinoma. Ann Surg Oncol 2013; 20 (11): 3596-3602

[122] Amini $\mathrm{N}$ et al. Temporal trends in liver-directed therapy of patients with intrahepatic cholangiocarcinoma in the United States: a populationbased analysis. J Surg Oncol 2014; 110 (2): 163-170

[123] Butros SR et al. Radiofrequency ablation of intrahepatic cholangiocarcinoma: feasability, local tumor control, and long-term outcome. Clin Imaging 2014; 38 (4): 490-494

[124] Fu Y et al. Radiofrequency ablation in the management of unresectable intrahepatic cholangiocarcinoma. J Vasc Interv Radiol 2012; 23 (5): 642-649

[125] Han K et al. Radiofrequency ablation in the treatment of unresectable intrahepatic cholangiocarcinoma: systematic review and meta-analysis. J Vasc Interv Radiol 2015; 26 (7): 943-948

[126] Kolarich AR et al. Non-surgical management of patients with intrahepatic cholangiocarcinoma in the United States, 2004-2015: an NCDB analysis. J Gastrointest Oncol 2018; 9 (3): 536-545

[127] Takahashi EA et al. Thermal ablation of intrahepatic cholangiocarcinoma: Safety, efficacy, and factors affecting local tumor progression. Abdom Radiol (NY) 2018; 43 (12): 3487-3492

[128] Kim JH et al. Radiofrequency ablation for recurrent intrahepatic cholangiocarcinoma after curative resection. Eur J Radiol 2011; 80 (3): e221-e225

[129] Goldaracena N, Gorgen A, Sapisochin G. Current status of liver transplantation for cholangiocarcinoma. Liver Transpl 2018; 24 (2): 294303

[130] Facciuto ME et al. Tumors with intrahepatic bile duct differentiation in cirrhosis: implications on outcomes after liver transplantation. Transplantation 2015; 99 (1): 151-157

[131] Vilchez $V$ et al. Long-term outcome of patients undergoing liver transplantation for mixed hepatocellular carcinoma and cholangiocarcinoma: an analysis of the UNOS database. HPB (Oxford) 2016; 18 (1): 29-34

[132] Sapisochin G et al. Intrahepatic cholangiocarcinoma or mixed hepatocellular-cholangiocarcinoma in patients undergoing liver transplantation: a Spanish matched cohort multicenter study. Ann Surg 2014; 259 (5): 944-952 
[133] Sapisochin G et al. Liver transplantation for "very early" intrahepatic cholangiocarcinoma: International retrospective study supporting a prospective assessment. Hepatology 2016; 64 (4): 1178-1188

[134] Lunsford KE et al. Liver transplantation for locally advanced intrahepatic cholangiocarcinoma treated with neoadjuvant therapy: a prospective case-series. Lancet Gastroenterol Hepatol 2018; 3 (5): 337348

[135] Becker NS et al. Outcomes analysis for 280 patients with cholangiocarcinoma treated with liver transplantation over an 18-year period. J Gastrointest Surg 2008; 12 (1): 117-122

[136] Darwish Murad S et al. Efficacy of neoadjuvant chemoradiation, followed by liver transplantation, for perihilar cholangiocarcinoma at 12 US centers. Gastroenterology 2012; 143 (1): 88-98.e3; quiz e14

[137] Rosen CB, Heimbach JK, Gores G]. Surgery for cholangiocarcinoma: the role of liver transplantation. HPB (Oxford) 2008; 10 (3): 186-189

[138] Gulamhusein AF, Sanchez W. Liver transplantation in the management of perihilar cholangiocarcinoma. Hepat Oncol 2015; 2 (4): 409-421

[139] Ethun CG et al. Transplantation Versus Resection for Hilar Cholangiocarcinoma: An Argument for Shifting Treatment Paradigms for Resectable Disease. Ann Surg 2018; 267 (5): 797-805

[140] Mantel HT et al. Strict Selection Alone of Patients Undergoing Liver Transplantation for Hilar Cholangiocarcinoma Is Associated with Improved Survival. PLoS One 2016; 11 (6): e0156127

[141] Weber SM et al. Intrahepatic cholangiocarcinoma: expert consensus statement. HPB (Oxford) 2015; 17 (8): 669-680

[142] NCCN Guidelines ${ }^{\circledR}$ for Hepatobiliary Cancers Version 3. 2019

[143] Ray CE Jr et al. Metaanalysis of survival, complications, and imaging response following chemotherapy-based transarterial therapy in patients with unresectable intrahepatic cholangiocarcinoma. J Vasc Interv Radiol 2013; 24 (8): 1218-1226

[144] Koch C et al. Poor Prognosis of Advanced Cholangiocarcinoma: RealWorld Data from a Tertiary Referral Center. Digestion 2019; 101: 458465

[145] Gusani N] et al. Treatment of unresectable cholangiocarcinoma with gemcitabine-based transcatheter arterial chemoembolization (TACE): a single-institution experience. J Gastrointest Surg 2008; 12 (1): 129137

[146] Boehm LM et al. Comparative effectiveness of hepatic artery based therapies for unresectable intrahepatic cholangiocarcinoma. J Surg Oncol 2015; 111 (2): 213-220

[147] Kiefer MV et al. Chemoembolization of intrahepatic cholangiocarcinoma with cisplatinum, doxorubicin, mitomycin C, ethiodol, and polyvinyl alcohol: a 2-center study. Cancer 2011; 117 (7): 1498-1505

[148] Vogl T] et al. Transarterial chemoembolization in the treatment of patients with unresectable cholangiocarcinoma: Results and prognostic factors governing treatment success. Int J Cancer 2012; 131 (3): 733 740

[149] Cucchetti A et al. Improving patient selection for selective internal radiation therapy of intra-hepatic cholangiocarcinoma: A meta-regression study. Liver Int 2017; 37 (7): 1056-1064

[150] Gangi A et al. Intrahepatic Cholangiocarcinoma Treated with Transarterial Yttrium-90 Glass Microsphere Radioembolization: Results of a Single Institution Retrospective Study. J Vasc Interv Radiol 2018; 29 (8): 1101-1108

[151] Manceau $V$ et al. A MAA-based dosimetric study in patients with intrahepatic cholangiocarcinoma treated with a combination of chemotherapy and (90)Y-loaded glass microsphere selective internal radiation therapy. Eur J Nucl Med Mol Imaging 2018; 45 (10): 1731-1741

[152] Reimer $P$ et al. Prognostic Factors in Overall Survival of Patients with Unresectable Intrahepatic Cholangiocarcinoma Treated by Means of Yttrium-90 Radioembolization: Results in Therapy-Naïve Patients. Cardiovasc Intervent Radiol 2018; 41 (5): 744-752
[153] Yang L et al. Trans-arterial embolisation therapies for unresectable intrahepatic cholangiocarcinoma: a systematic review. J Gastrointest Oncol 2015; 6 (5): 570-88

[154] Koch C et al. Poor Prognosis of Advanced Cholangiocarcinoma: RealWorld Data from a Tertiary Referral Center. Digestion 2020; 101 (4): 458-465

[155] Hyder O et al. Intra-arterial therapy for advanced intrahepatic cholangiocarcinoma: a multi-institutional analysis. Ann Surg Oncol 2013; 20 (12): 3779-3786

[156] Marquardt S et al. Percutaneous hepatic perfusion (chemosaturation) with melphalan in patients with intrahepatic cholangiocarcinoma: European multicentre study on safety, short-term effects and survival. Eur Radiol 2019; 29 (4): 1882-1892

[157] Edeline J et al. Radioembolization Plus Chemotherapy for First-line Treatment of Locally Advanced Intrahepatic Cholangiocarcinoma: A Phase 2 Clinical Trial. JAMA Oncol 2019; 6 (1): 51-59

[158] Konstantinidis IT et al. Unresectable intrahepatic cholangiocarcinoma: Systemic plus hepatic arterial infusion chemotherapy is associated with longer survival in comparison with systemic chemotherapy alone. Cancer 2016; 122 (5): 758-765

[159] Al-Adra DP et al. Treatment of unresectable intrahepatic cholangiocarcinoma with yttrium-90 radioembolization: a systematic review and pooled analysis. Eur J Surg Oncol 2015; 41 (1): 120-127

[160] Wronka KM et al. Relevance of Preoperative Hyperbilirubinemia in Patients Undergoing Hepatobiliary Resection for Hilar Cholangiocarcinoma. J Clin Med 2019; 8 (4): 458

[161] Al Mahjoub A et al. Preoperative Biliary Drainage in Patients with Resectable Perihilar Cholangiocarcinoma: Is Percutaneous Transhepatic Biliary Drainage Safer and More Effective than Endoscopic Biliary Drainage? A Meta-Analysis. J Vasc Interv Radiol 2017; 28 (4): 576-582

[162] Hameed A et al. Percutaneous vs. endoscopic pre-operative biliary drainage in hilar cholangiocarcinoma - a systematic review and metaanalysis. HPB (Oxford) 2016; 18 (5): 400-410

[163] Coelen RJS et al. Endoscopic versus percutaneous biliary drainage in patients with resectable perihilar cholangiocarcinoma: a multicentre, randomised controlled trial. Lancet Gastroenterol Hepatol 2018; 3 (10): 681-690

[164] Ba $Y$ et al. Percutaneous transhepatic biliary drainage may be the preferred preoperative drainage method in hilar cholangiocarcinoma. Endosc Int Open 2020; 8 (2): E203-E210

[165] Maeda T et al. Preoperative course of patients undergoing endoscopic nasobiliary drainage during the management of resectable perihilar cholangiocarcinoma. J Hepatobiliary Pancreat Sci 2019; 26 (8): 341347

[166] Nakai $Y$ et al. Multicenter study of endoscopic preoperative biliary drainage for malignant hilar biliary obstruction: E-POD hilar study. J Gastroenterol Hepatol 2018; 33 (5): 1146-1153

[167] Komaya K et al. Verification of the oncologic inferiority of percutaneous biliary drainage to endoscopic drainage: A propensity score matching analysis of resectable perihilar cholangiocarcinoma. Surgery 2017; 161 (2): 394-404

[168] Kim KM et al. A Comparison of Preoperative Biliary Drainage Methods for Perihilar Cholangiocarcinoma: Endoscopic versus Percutaneous Transhepatic Biliary Drainage. Gut Liver 2015; 9 (6): 791-799

[169] Kennedy T] et al. Role of preoperative biliary drainage of liver remnant prior to extended liver resection for hilar cholangiocarcinoma. HPB (Oxford) 2009; 11 (5): 445-451

[170] Miura S et al. Preoperative biliary drainage of the hepatic lobe to be resected does not affect liver hypertrophy after percutaneous transhepatic portal vein embolization. Surg Endosc 2020; 34 (2): 667-674

[171] Hintze RE et al. Magnetic resonance cholangiopancreatography-guided unilateral endoscopic stent placement for Klatskin tumors. Gastrointest Endosc 2001; 53 (1): 40-46 
[172] Abraham NS, Barkun JS, Barkun AN. Palliation of malignant biliary obstruction: a prospective trial examining impact on quality of life. Gastrointest Endosc 2002; 56 (6): 835-841

[173] Paik WH et al. Palliative treatment with self-expandable metallic stents in patients with advanced type III or IV hilar cholangiocarcinoma: a percutaneous versus endoscopic approach. Gastrointest Endosc 2009; 69 (1): 55-62

[174] Saluja SS et al. Endoscopic or percutaneous biliary drainage for gallbladder cancer: a randomized trial and quality of life assessment. Clin Gastroenterol Hepatol 2008; 6 (8): 944-950.e3

[175] Schima W et al. Biliary Wallstent endoprosthesis in malignant hilar obstruction: long-term results with regard to the type of obstruction. Clin Radiol 1997; 52 (3): 213-219

[176] Rees J et al. The outcomes of biliary drainage by percutaneous transhepatic cholangiography for the palliation of malignant biliary obstruction in England between 2001 and 2014: a retrospective cohort study. BMJ Open 2020; 10 (1): e033576

[177] Uberoi R et al. British Society of Interventional Radiology: Biliary Drainage and Stenting Registry (BDSR). Cardiovasc Intervent Radiol 2012; 35 (1): 127-138

[178] Smith AC et al. Randomised trial of endoscopic stenting versus surgical bypass in malignant low bileduct obstruction. Lancet 1994; 344: 16551660

[179] Speer AG et al. Randomised trial of endoscopic versus percutaneous stent insertion in malignant obstructive jaundice. Lancet 1987; 2: 5762

[180] Almadi MA, Barkun A, Martel M. Plastic vs. Self-Expandable Metal Stents for Palliation in Malignant Biliary Obstruction: A Series of MetaAnalyses. Am J Gastroenterol 2017; 112 (2): 260-273

[181] Lee TH et al. Prospective comparison of endoscopic bilateral stent-instent versus stent-by-stent deployment for inoperable advanced malignant hilar biliary stricture. Gastrointest Endosc 2019; 90 (2): 222-230

[182] Sharaiha RZ et al. Endoscopic ultrasound-guided biliary drainage versus percutaneous transhepatic biliary drainage: predictors of successful outcome in patients who fail endoscopic retrograde cholangiopancreatography. Surg Endosc 2016; 30 (12): 5500-5505

[183] Paik WH et al. EUS-Guided Biliary Drainage Versus ERCP for the Primary Palliation of Malignant Biliary Obstruction: A Multicenter Randomized Clinical Trial. Am J Gastroenterol 2018; 113 (7): 987-997

[184] Bang JY et al. Stent placement by EUS or ERCP for primary biliary decompression in pancreatic cancer: a randomized trial (with videos). Gastrointest Endosc 2018; 88 (1): 9-17

[185] Dumonceau JM et al. Endoscopic biliary stenting: indications, choice of stents, and results: European Society of Gastrointestinal Endoscopy (ESGE) Clinical Guideline - Updated October 2017. Endoscopy 2018; 50 (9): 910-930

[186] Moole $\mathrm{H}$ et al. Endoscopic versus Percutaneous Biliary Drainage in Palliation of Advanced Malignant Hilar Obstruction: A Meta-Analysis and Systematic Review. Can J Gastroenterol Hepatol 2016; 2016: 4726078

[187] Zhao XQ et al. Comparison of percutaneous transhepatic biliary drainage and endoscopic biliary drainage in the management of malignant biliary tract obstruction: a meta-analysis. Dig Endosc 2015; 27 (1): 137-145

[188] Born $\mathrm{P}$ et al. Long-term results of percutaneous transhepatic biliary drainage for benign and malignant bile duct strictures. Scand J Gastroenterol 1998; 33 (5): 544-549

[189] De Palma GD et al. Unilateral versus bilateral endoscopic hepatic duct drainage in patients with malignant hilar biliary obstruction: results of a prospective, randomized, and controlled study. Gastrointest Endosc 2001; 53 (6): 547-553

[190] Chang WH, Kortan P, Haber GB. Outcome in patients with bifurcation tumors who undergo unilateral versus bilateral hepatic duct drainage. Gastrointest Endosc 1998; 47 (5): 354-362
[191] Bulajic M et al. Clinical outcome in patients with hilar malignant strictures type II Bismuth-Corlette treated by minimally invasive unilateral versus bilateral endoscopic biliary drainage. Hepatobiliary Pancreat Dis Int 2012; 11 (2): 209-214

[192] Cheng JL et al. Endoscopic palliation of patients with biliary obstruction caused by nonresectable hilar cholangiocarcinoma: efficacy of selfexpandable metallic Wallstents. Gastrointest Endosc 2002; 56 (1): 33 39

[193] Vienne A et al. Prediction of drainage effectiveness during endoscopic stenting of malignant hilar strictures: the role of liver volume assessment. Gastrointest Endosc 2010; 72 (4): 728-735

[194] Harvey PR et al. Higher volume providers are associated with improved outcomes following ERCP for the palliation of malignant biliary obstruction. EClinicalMedicine 2020; 18: 100212

[195] Tal AO et al. Intraductal endoscopic radiofrequency ablation for the treatment of hilar non-resectable malignant bile duct obstruction. World J Gastrointest Endosc 2014; 6 (1): 13-19

[196] Moole $\mathrm{H}$ et al. Success of photodynamic therapy in palliating patients with nonresectable cholangiocarcinoma: A systematic review and meta-analysis. World J Gastroenterol 2017; 23 (7): 1278-1288

[197] Zoepf T et al. Photodynamic therapy with 5-aminolevulinic acid is not effective in bile duct cancer. Gastrointest Endosc 2001; 54 (6): $763-$ 766

[198] Yang J et al. Efficacy and safety of endoscopic radiofrequency ablation for unresectable extrahepatic cholangiocarcinoma: a randomized trial. Endoscopy 2018; 50 (8): 751-760

[199] Ortner ME et al. Successful photodynamic therapy for nonresectable cholangiocarcinoma: a randomized prospective study. Gastroenterology 2003; 125 (5): 1355-1363

[200] Zoepf T et al. Palliation of nonresectable bile duct cancer: improved survival after photodynamic therapy. Am J Gastroenterol 2005; 100 (11): 2426-2430

[201] Pereira SP et al. PHOTOSTENT-02: porfimer sodium photodynamic therapy plus stenting versus stenting alone in patients with locally advanced or metastatic biliary tract cancer. ESMO Open 2018; 3 (5): e000379

[202] Gonzalez-Carmona MA et al. Combined photodynamic therapy with systemic chemotherapy for unresectable cholangiocarcinoma. Aliment Pharmacol Ther 2019; 49 (4): 437-447

[203] Wentrup R et al. Photodynamic Therapy Plus Chemotherapy Compared with Photodynamic Therapy Alone in Hilar Nonresectable Cholangiocarcinoma. Gut Liver 2016; 10 (3): 470-475

[204] Strand DS et al. ERCP-directed radiofrequency ablation and photodynamic therapy are associated with comparable survival in the treatment of unresectable cholangiocarcinoma. Gastrointest Endosc 2014; 80 (5): 794-804

[205] Dolak W et al. Photodynamic therapy with polyhematoporphyrin for malignant biliary obstruction: A nationwide retrospective study of 150 consecutive applications. United European Gastroenterol J 2017; 5 (1): 104-110

[206] Kahaleh M et al. Unresectable cholangiocarcinoma: comparison of survival in biliary stenting alone versus stenting with photodynamic therapy. Clin Gastroenterol Hepatol 2008; 6 (3): 290-297

[207] Ben-Josef E et al. Phase II trial of high-dose conformal radiation therapy with concurrent hepatic artery floxuridine for unresectable intrahepatic malignancies. J Clin Oncol 2005; 23 (34): 8739-8747

[208] Brunner TB et al. Stereotactic body radiotherapy dose and its impact on local control and overall survival of patients for locally advanced intrahepatic and extrahepatic cholangiocarcinoma. Radiother Oncol 2019; 132: 42-47

[209] Tao R et al. Ablative Radiotherapy Doses Lead to a Substantial Prolongation of Survival in Patients With Inoperable Intrahepatic Cholangio- 
carcinoma: A Retrospective Dose Response Analysis. J Clin Oncol 2016; 34 (3): 219-226

[210] Lee J et al. Efficacy of stereotactic body radiotherapy for unresectable or recurrent cholangiocarcinoma: a meta-analysis and systematic review. Strahlenther Onkol 2019; 195 (2): 93-102

[211] Frakulli R et al. Stereotactic body radiation therapy in cholangiocarcinoma: a systematic review. Br J Radiol 2019; 92: 20180688

[212] Barney BM et al. Clinical outcomes and toxicity using stereotactic body radiotherapy (SBRT) for advanced cholangiocarcinoma. Radiat Oncol 2012; 7: 67

[213] Tse RV et al. Phase I study of individualized stereotactic body radiotherapy for hepatocellular carcinoma and intrahepatic cholangiocarcinoma. J Clin Oncol 2008; 26 (4): 657-664

[214] Weiner AA et al. Stereotactic body radiotherapy for primary hepatic malignancies - Report of a phase I/II institutional study. Radiother Oncol 2016; 121 (1): 79-85

[215] Kopek N et al. Stereotactic body radiotherapy for unresectable cholangiocarcinoma. Radiother Oncol 2010; 94 (1): 47-52

[216] Hong TS et al. Multi-Institutional Phase II Study of High-Dose Hypofractionated Proton Beam Therapy in Patients With Localized, Unresectable Hepatocellular Carcinoma and Intrahepatic Cholangiocarcinoma. J Clin Oncol 2016; 34 (5): 460-468

[217] Schnapauff D et al. Computed tomography-guided interstitial HDR brachytherapy (CT-HDRBT) of the liver in patients with irresectable intrahepatic cholangiocarcinoma. Cardiovasc Intervent Radiol 2012; 35 (3): 581-587

[218] Vogel A et al. The diagnosis and treatment of cholangiocarcinoma. Dtsch Arztebl Int 2014; 111 (44): 748-754

[219] Horgan AM et al. Adjuvant therapy in the treatment of biliary tract cancer: a systematic review and meta-analysis. J Clin Oncol 2012; 30 (16): 1934-1940

[220] Edeline J et al. Gemox versus surveillance following surgery of localized biliary tract cancer: Results of the PRODIGE 12-ACCORD 18 (UNICANCER GI) phase III trial. Journal of Clinical Oncology 2017; 35 (4): 225225

[221] Stein A et al. Adjuvant chemotherapy with gemcitabine and cisplatin compared to observation after curative intent resection of cholangiocarcinoma and muscle invasive gallbladder carcinoma (ACTICCA-1 trial) - a randomized, multidisciplinary, multinational phase III trial. BMC Cancer 2015; 15: 564

[222] Valle J et al. Cisplatin plus gemcitabine versus gemcitabine for biliary tract cancer. N Engl J Med 2010; 362 (14): 1273-1281

[223] Shroff RT et al. Gemcitabine, Cisplatin, and nab-Paclitaxel for the Treatment of Advanced Biliary Tract Cancers: A Phase 2 Clinical Trial. JAMA Oncol 2019; 5 (6): 824-830

[224] Okusaka T et al. Gemcitabine alone or in combination with cisplatin in patients with biliary tract cancer: a comparative multicentre study in Japan. Br J Cancer 2010; 103 (4): 469-474

[225] Valle JW et al. Cisplatin and gemcitabine for advanced biliary tract cancer: a meta-analysis of two randomised trials. Ann Oncol 2014; 25 (2): 391-398

[226] Park JO et al. Gemcitabine Plus Cisplatin for Advanced Biliary Tract Cancer: A Systematic Review. Cancer Res Treat 2015; 47 (3): 343-361

[227] Valle JW et al. Biliary cancer: ESMO Clinical Practice Guidelines for diagnosis, treatment and follow-up. Ann Oncol 2016; 27 (Suppl. 5): v28-v37

[228] Lamarca A et al. ABC-06 | A randomised phase III, multi-centre, openlabel study of active symptom control (ASC) alone or ASC with oxaliplatin / 5-FU chemotherapy (ASC+mFOLFOX) for patients (pts) with locally advanced / metastatic biliary tract cancers ( $A B C$ ) previouslytreated with cisplatin/gemcitabine (CisGem) chemotherapy. Journal of Clinical Oncology 2019; 37 (15): 4003-4003
[229] Lamarca A et al. Second-line chemotherapy in advanced biliary cancer: a systematic review. Ann Oncol 2014; 25 (12): 2328-2338

[230] Walter T et al. Feasibility and benefits of second-line chemotherapy in advanced biliary tract cancer: a large retrospective study. Eur J Cancer 2013; 49 (2): 329-335

[231] Brieau B et al. Second-line chemotherapy for advanced biliary tract cancer after failure of the gemcitabine-platinum combination: A large multicenter study by the Association des Gastro-Entérologues Oncologues. Cancer 2015; 121 (18): 3290-3297

[232] Abou-Alfa GK et al. Pemigatinib for previously treated, locally advanced or metastatic cholangiocarcinoma: a multicentre, open-label, phase 2 study. Lancet Oncol 2020; 21 (5): 671-684

[233] Valle JW et al. New Horizons for Precision Medicine in Biliary Tract Cancers. Cancer Discov 2017; 7 (9): 943-962

[234] Marabelle A et al. Efficacy of Pembrolizumab in Patients With Noncolorectal High Microsatellite Instability/Mismatch Repair-Deficient Cancer: Results From the Phase II KEYNOTE-158 Study. Journal of Clinical Oncology 0 (): JCO.19.02105

[235] Le DT et al. Mismatch repair deficiency predicts response of solid tumors to PD-1 blockade. Science 2017: 357: 409-413

[236] Le DT et al. PD-1 Blockade in Tumors with Mismatch-Repair Deficiency. N Engl J Med 2015; 372 (26): 2509-2520

[237] Goeppert B et al. Mismatch repair deficiency is a rare but putative therapeutically relevant finding in non-liver fluke associated cholangiocarcinoma. Br J Cancer 2019; 120 (1): 109-114

[238] Katoh M. Fibroblast growth factor receptors as treatment targets in clinical oncology. Nat Rev Clin Oncol 2018; 16: 105-122

[239] Jain A et al. Cholangiocarcinoma With FGFR Genetic Aberrations: A Unique Clinical Phenotype. JCO Precision Oncology 2018; (2): 1-12

[240] Sia D et al. Massive parallel sequencing uncovers actionable FGFR2PPHLN1 fusion and ARAF mutations in intrahepatic cholangiocarcinoma. Nat Commun 2015; 6: 6087

[241] Javle M et al. Phase II Study of BGJ398 in Patients With FGFR-Altered Advanced Cholangiocarcinoma. J Clin Oncol 2018; 36 (3): 276-282

[242] Mazzaferro V et al. Derazantinib (ARQ 087) in advanced or inoperable FGFR2 gene fusion-positive intrahepatic cholangiocarcinoma. $\mathrm{Br}$ J Cancer 2019; 120 (2): 165-171

[243] Bahleda R et al. Multicenter Phase I Study of Erdafitinib (JNJ-42756493), Oral Pan-Fibroblast Growth Factor Receptor Inhibitor, in Patients with Advanced or Refractory Solid Tumors. Clin Cancer Res 2019; 25 (16): 4888-4897

[244] Abou-Alfa GK et al. Pemigatinib for previously treated, locally advanced or metastatic cholangiocarcinoma: a multicentre, open-label, phase 2 study. The Lancet Oncology 2020; 21 (5): 671-684

[245] Cocco E, Scaltriti M, Drilon A. NTRK fusion-positive cancers and TRK inhibitor therapy. Nat Rev Clin Oncol 2018; 15 (12): 731-747

[246] Solomon JP et al. NTRK fusion detection across multiple assays and 33997 cases: diagnostic implications and pitfalls. Mod Pathol 2019; 33: $38-46$

[247] Ross JS et al. New routes to targeted therapy of intrahepatic cholangiocarcinomas revealed by next-generation sequencing. Oncologist 2014; 19 (3): 235-242

[248] Drilon A et al. Efficacy of Larotrectinib in TRK Fusion-Positive Cancers in Adults and Children. N Engl J Med 2018; 378 (8): 731-739

[249] Oh DY, Bang Y]. HER2-targeted therapies - a role beyond breast cancer. Nat Rev Clin Oncol 2019; 17: 33-48

[250] Neyaz A et al. Investigation of targetable predictive and prognostic markers in gallbladder carcinoma. J Gastrointest Oncol 2018; 9 (1): 111-125

[251] Javle $M$ et al. HER2/neu-directed therapy for biliary tract cancer. J Hematol Oncol 2015; 8: 58 
[252] Czink E et al. [Durable remission under dual HER2 blockade with Trastuzumab and Pertuzumab in a patient with metastatic gallbladder cancer]. Z Gastroenterol 2016; 54 (5): 426-430

[253] Hyman DM et al. Vemurafenib in Multiple Nonmelanoma Cancers with BRAF V600 Mutations. N Engl J Med 2015; 373 (8): 726-736

[254] Salama AKS et al. Dabrafenib and trametinib in patients with tumors with BRAF V600E/K mutations: Results from the molecular analysis for therapy choice (MATCH) Arm H. Journal of Clinical Oncology 2019; 37 (15): 3002

[255] Lavingia V, Fakih M. Impressive response to dual BRAF and MEK inhibition in patients with BRAF mutant intrahepatic cholangiocarcinoma-2 case reports and a brief review. J Gastrointest Oncol 2016; 7 (6): E98E102

[256] Kocsis ] et al. Combined dabrafenib and trametinib treatment in a case of chemotherapy-refractory extrahepatic BRAF V600E mutant cholangiocarcinoma: dramatic clinical and radiological response with a confusing synchronic new liver lesion. J Gastrointest Oncol 2017; 8 (2): E32-E38

[257] Bunyatov T et al. Personalised approach in combined treatment of cholangiocarcinoma: a case report of healing from cholangiocellular carcinoma at stage IV. J Gastrointest Oncol 2019; 10 (4): 815-820

[258] Abou-Alfa GK et al. Ivosidenib in IDH1-mutant, chemotherapyrefractory cholangiocarcinoma (ClarlDHy): a multicentre, randomised, double-blind, placebo-controlled, phase 3 study. The Lancet Oncology 2020; 21 (6): 796-807

[259] Leitlinienprogramm Onkologie (Deutsche Krebsgesellschaft, D.K., AWMF). Entwicklung von leitlinienbasierten Qualitätsindikatoren. Methodenpapier für das Leitlinienprogramm Onkologie, Version 2.1.
2017. Available from: http://www.leitlinienprogramm-onkologie.de/ methodik/informationen-zur-methodik/

[260] Celotti A et al. Preoperative biliary drainage in hilar cholangiocarcinoma: Systematic review and meta-analysis. Eur J Surg Oncol 2017; 43 (9): 1628-1635

[261] Ramanathan R et al. Preoperative Biliary Drainage Is Associated with Increased Complications After Liver Resection for Proximal Cholangiocarcinoma. J Gastrointest Surg 2018; 22 (11): 1950-1957

[262] Cai Y et al. Preoperative biliary drainage versus direct surgery for perihilar cholangiocarcinoma: A retrospective study at a single center. Biosci Trends 2017; 11 (3): 319-325

[263] Farges O et al. Multicentre European study of preoperative biliary drainage for hilar cholangiocarcinoma. Br J Surg 2013; 100 (2): 274 283

[264] Xiong J] et al. Preoperative biliary drainage in patients with hilar cholangiocarcinoma undergoing major hepatectomy. World J Gastroenterol 2013; 19 (46): 8731-8739

[265] Wang L et al. A systematic review of the comparison of the incidence of seeding metastasis between endoscopic biliary drainage and percutaneous transhepatic biliary drainage for resectable malignant biliary obstruction. World J Surg Oncol 2019; 17 (1): 116

[266] Kishi Y et al. The type of preoperative biliary drainage predicts shortterm outcome after major hepatectomy. Langenbecks Arch Surg 2016; 401 (4): 503-511

[267] Sangchan A et al. Efficacy of metal and plastic stents in unresectable complex hilar cholangiocarcinoma: a randomized controlled trial. Gastrointest Endosc 2012; 76 (1): 93-99 